The impact of recent advances in laboratory astrophysics on our understanding of the cosmos

This article has been downloaded from IOPscience. Please scroll down to see the full text article.

2012 Rep. Prog. Phys. 75036901

(http://iopscience.iop.org/0034-4885/75/3/036901)

View the table of contents for this issue, or go to the journal homepage for more

Download details:

IP Address: 129.15.30.75

The article was downloaded on 22/02/2012 at 17:44

Please note that terms and conditions apply. 


\title{
The impact of recent advances in laboratory astrophysics on our understanding of the cosmos
}

\author{
D W Savin ${ }^{1}$, N S Brickhouse ${ }^{2}$, J J Cowan ${ }^{3}$, R P Drake ${ }^{4}$, S R Federman ${ }^{5}$, \\ G J Ferland ${ }^{6}$, A Frank ${ }^{7}$, M S Gudipati ${ }^{8}$, W C Haxton ${ }^{9}$, E Herbst ${ }^{10}$, S Profumo ${ }^{11}$, \\ F Salama ${ }^{12}$, L M Ziurys ${ }^{13}$ and E G Zweibel ${ }^{14}$ \\ ${ }^{1}$ Columbia Astrophysics Laboratory, Columbia University, New York, NY 10027, USA \\ ${ }^{2}$ Harvard-Smithsonian Center for Astrophysics, 60 Garden Street, Cambridge, MA 02138, USA \\ ${ }^{3}$ Homer L Dodge Department of Physics and Astronomy, University of Oklahoma, Norman, OK 73019, USA \\ ${ }^{4}$ Department of Atmospheric, Oceanic and Space Sciences, University of Michigan, Ann Arbor, MI 48109, USA \\ ${ }^{5}$ Department of Physics and Astronomy, University of Toledo, Toledo, OH 43606, USA \\ ${ }^{6}$ Department of Physics, University of Kentucky, Lexington, KY 40506, USA \\ ${ }^{7}$ Department of Physics and Astronomy, University of Rochester, Rochester, NY 14627, USA \\ ${ }^{8}$ Science Division, Jet Propulsion Laboratory, California Institute of Technology, Pasadena, CA 91109, USA \\ ${ }^{9}$ Department of Physics, University of California, Berkeley, and Lawrence Berkeley National Laboratory, \\ Berkeley, CA 97420, USA \\ ${ }^{10}$ Departments of Chemistry, Astronomy and Physics, University of Virginia, Charlottesville, VA 22904, USA \\ ${ }^{11}$ Department of Physics, ISB 325, University of California, 1156 High Street, Santa Cruz, CA 95064, USA \\ ${ }^{12}$ Space Science Division, NASA Ames Research Center, Moffett Field, CA 94035, USA \\ ${ }^{13}$ Departments of Chemistry and Astronomy, Arizona Radio Observatory and Steward Observatory, University of \\ Arizona, Tucson, AZ 85721, USA \\ ${ }^{14}$ Departments of Astronomy and Physics, University of Wisconsin, 6281 Chamberlain Hall, 475 North Charter \\ Street, Madison, WI 53706, USA
}

Received 18 October 2009, in final form 8 November 2011

Published 22 February 2012

Online at stacks.iop.org/RoPP/75/036901

\begin{abstract}
An emerging theme in modern astrophysics is the connection between astronomical observations and the underlying physical phenomena that drive our cosmos. Both the mechanisms responsible for the observed astrophysical phenomena and the tools used to probe such phenomena- the radiation and particle spectra we observe-have their roots in atomic, molecular, condensed matter, plasma, nuclear and particle physics. Chemistry is implicitly included in both molecular and condensed matter physics. This connection is the theme of the present report, which provides a broad, though non-exhaustive, overview of progress in our understanding of the cosmos resulting from recent theoretical and experimental advances in what is commonly called laboratory astrophysics. This work, carried out by a diverse community of laboratory astrophysicists, is increasingly important as astrophysics transitions into an era of precise measurement and high fidelity modeling.
\end{abstract}

(Some figures may appear in colour only in the online journal)

This article was invited by S Ritz.

\section{Contents}

1. Introduction

2. Planetary systems and star formation 2.1. Atomic physics
2.2. Molecular physics

2.3. Condensed matter physics

2.4. Plasma physics 
3. Stars and stellar evolution

3.1. Atomic physics

3.2. Molecular physics

3.3. Condensed matter physics

3.4. Plasma physics

3.5. Nuclear physics

4. The galactic neighborhood

4.1. Atomic physics

4.2. Molecular physics

4.3. Plasma physics

5. Galaxies across time
5.1. Atomic physics

5.2. Molecular physics 22

6. Cosmology and fundamental physics 22

6.1. Atomic physics 22

6.2. Molecular physics 23

6.3. Nuclear physics 23

6.4. Particle physics 24

7. Discussion and outlook for the future 26

Acknowledgments $\quad 27$

Appendix A. Acronyms 27

References $\quad 28$

\section{Introduction}

Laboratory astrophysics and complementary theoretical calculations are the foundations of astronomy and astrophysics and will remain so into the foreseeable future. The impact of laboratory astrophysics ranges from the scientific conception for ground-based, airborne and space-based observatories, all the way through to the scientific return of these projects and missions. It is our understanding of the underlying physical processes and the measurement or calculation of critical physical parameters that allows us to address fundamental questions in astronomy and astrophysics.

The field of laboratory astrophysics comprises both theoretical and experimental studies of the underlying physics that produce the observed astrophysical processes. We have identified six areas of physics as relevant to astronomy and astrophysics ${ }^{15}$. Astronomy is an observational science focused primarily on detecting photons generated by atomic, molecular and condensed matter physics. Chemistry is implicitly included here as part of molecular and condensed matter physics. Our understanding of the universe also relies on knowledge of the evolution of matter (nuclear and particle physics) and of the dynamical processes shaping it (plasma physics). Planetary science, involving in situ measurements of solar system bodies, requires knowledge from atomic, molecular, condensed matter and plasma physics. Hence, our quest to understand the cosmos rests firmly on scientific knowledge in six areas: atomic, molecular, condensed matter, plasma, nuclear and particle physics.

Here we review recent advances in our astrophysical understanding of the cosmos arising from work in laboratory astrophysics. We focus primarily on the past decade. Our work complements that of previous reviews on laboratory astrophysics in atomic physics (Beiersdorfer 2003, Kallman and Palmeri 2007, International Astronomical Union (IAU) Commission 14 2011), molecular physics (Salama 1999, Tielens 2005, Herbst and van Dishoeck 2009, IAU Commission 14 2011), condensed matter physics (Draine 2003, Whittet 2003), plasma physics (Drake 1999, Remington et al 2006, Zweibel and Yamada 2009, Yamada et al 2010), nuclear physics (Käppeler et al 2011, Wiescher et al 2010,

15 The authors comprise past and current members of the American Astronomical Society Working Group on Laboratory Astrophysics.
Adelberger et al 2011) and particle physics (Grupen 2005, Aprile and Profumo 2009).

Because laboratory astrophysics, as implied by its name, is astrophysically motivated, we have structured our report into five broad categories which blanket the field of astronomy and astrophysics. This helps us to bring out the synergy between the various subareas of laboratory astrophysics. The specific categories are as follows: planetary systems and star formation (section 2), stars and stellar evolution (section 3), the galactic neighborhood (section 4), galaxies across time (section 5) and cosmology and fundamental physics (section 6). This structure parallels the scientific divisions used by the recent US National Research Council Astro 2010 Survey on Astronomy and Astrophysics (Blandford et al 2010a). These five sections are further subdivided into relevant subareas of laboratory astrophysics. Space limitations prevent these subsections from being exhaustive. Rather they are aimed at giving the reader an overview of recent successes in the field and appropriate citations to provide entry into the relevant research. We conclude with a brief discussion and outlook for the future in section 7 .

\section{Planetary systems and star formation}

Planetary systems and star formation encompass 'solar system bodies (other than the Sun) and extrasolar planets, debris disks, exobiology, the formation of individual stars, protostellar and protoplanetary disks, molecular clouds and the cold ISM ${ }^{16}$ (interstellar medium), dust, and astrochemistry' (Blandford et al 2010a).

\subsection{Atomic physics}

2.1.1. Young late-type stars. In accreting stellar objects with strong magnetic fields (such as young late-type stars, x-ray binaries with neutron stars and magnetic cataclysmic variables $(\mathrm{CVs})$ ), the stellar magnetic field truncates the accretion disk and channels the accreting material toward a 'hot spot' near the pole of the star (Konigl 1991). This material accelerates in the gravitational field of the star, reaching supersonic velocities and producing a shock which emits in x-rays. For low-mass young stars, the free-fall velocity (the maximum velocity obtained by material accelerated from infinity) is

${ }^{16} \mathrm{~A}$ list of acronyms used throughout the text is given in the appendix. 


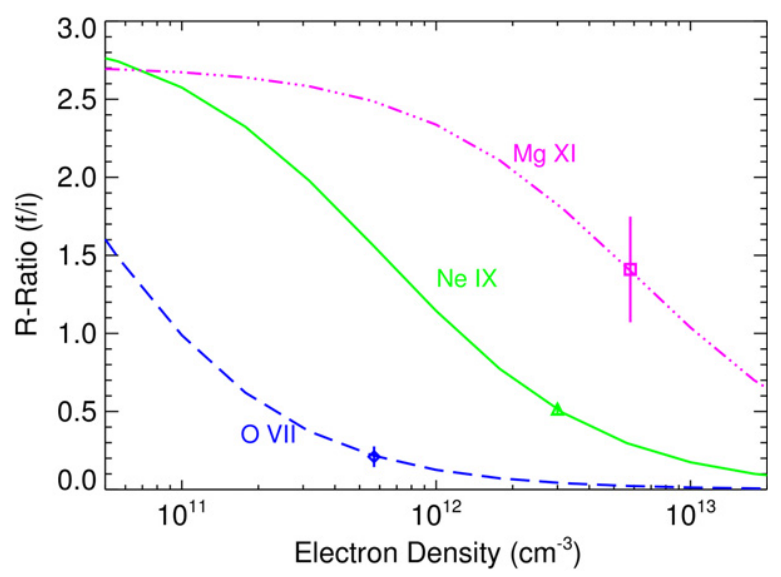

Figure 1. Theoretical He-like ion forbidden to intercombination line $R$-ratios (f/i) as a function of density (curves) overplotted with the observed line ratios from the Chandra spectrum of the young star TW Hya (points with $1 \sigma$ error bars). As discussed in the text, the electron temperature and density are determined using accurate atomic data. Accretion shock models are in good agreement with the Ne IX and Mg XI densities and temperatures at the shock front. However, the models fail to match the observed $\mathrm{O}$ vII density, from Brickhouse et al (2010).

$\sim 500 \mathrm{~km} \mathrm{~s}^{-1}$ and the expected shock temperatures are around a few MK (Calvet and Gullbring 1998, Kastner et al 2002). High electron densities of $\sim 10^{13} \mathrm{~cm}^{-3}$ are also expected at the shock, assuming the ram pressure of the gas balances the stellar atmospheric pressure. Electron temperature and density diagnostics are available using He-like lines observed in X-ray spectra from $\mathrm{O}$ VII, $\mathrm{Ne}$ IX and Mg XI. However, atomic theoretical models of these diagnostic lines have only recently become accurate enough to test shock models (Chen et al 2006, Smith et al 2009). Applying the new atomic data to a long observation (500 ks) of TW Hya with the Chandra X-ray Observatory High Energy Transmission Grating, Brickhouse et al (2010) showed that the shock models work well at the shock front. But, again using accurate diagnostics, the standard model fails to describe the spectra of the post-shock cooling gas. In the standard model, the electron density increases as the shocked gas cools and recombines, but instead the opposite is observed: the observed density of the cooler $\mathrm{O}_{\mathrm{VII}}$ is lower than that of the hotter $\mathrm{Ne}$ IX by a factor of 4 (figure 1) and lower than the model prediction by a factor of 7 . In contradiction to the post-shock models of cooling and 'settling' gas, the shock heats a significant mass of stellar atmosphere to soft x-ray emitting temperatures. This discovery has implications for coronal heating and wind driving in the presence of accretion.

2.1.2. Cometary $x$-ray emission. The discovery of x-ray and extreme ultraviolet emission from comet C/Hyakutake (Lisse et al 1996) was a great surprise. The subsequent identification of the emission mechanism as charge exchange with the highly charged ions of the solar wind (Cravens 1997, Krasnopolsky et al 1997) has led to tremendous progress in understanding the solar system (see Bhardwaj et al (2007)). High spectral resolution observations revealed the classic signature of charge exchange, namely dominant features from high angular momentum states and thus high principal quantum levels
(Kharchenko and Dalgarno 2000, Krasnopolsky and Mumma 2001, Lisse et al 2001). Calculations and experiments of charge exchange are now incorporated into x-ray studies of the interaction between the solar wind and planets, comets and the heliosphere. Cravens et al (2001) predicted that charge exchange of solar wind ions in the heliosphere and geocorona could produce half the soft $\mathrm{x}$-ray background. The longstanding mystery of the soft $\mathrm{x}$-ray background (and one of the key goals of Chandra) is now being solved: perhaps all or most of this background comes from charge exchange of the solar wind within the heliosphere (Koutroumpa et al 2006), with important implications for the interstellar environment surrounding the solar system. Experimental measurements continue to be important for quantitative analysis of charge exchange spectra (see, e.g., Beiersdorfer et al (2000), Greenwood et al (2000), Beiersdorfer et al (2003) and Otranto and Olson (2011)). Dennerl (2010) provides a good review of this field.

2.1.3. Exoplanetary discovery. Nearly 500 planets around other stars have been discovered to date using a variety of techniques, with many more expected from the Kepler mission (Borucki et al 2010). The $\sim 100$ exoplanets that transit their host stars are scientifically invaluable since both the mass and radius of the planet can be determined (see, e.g., Maxted et al (2010)). Transit searches involve two main stages: repeated photometric detection of transits of acceptable depth and duration, followed by spectroscopic confirmation. The first exoplanet discovered by the transit method exploited a detailed stellar atmosphere model of the star, cross-correlated with the observed spectra, in order to determine radial velocities (Konacki et al 2003, 2004, Sasselov 2003). This approach has now become a standard tool in the field, with many refinements added (Torres et al 2011). These atmosphere models incorporate an enormous database of atomic and molecular line transitions (Kurucz and Bell 1995, Castelli et al 1997). The precision in radial velocity that can be achieved depends strongly on the fraction of spectral lines in the model that match the observation; hence, ongoing efforts to improve the line lists go hand in hand with continuing discoveries in this field.

\subsection{Molecular physics}

2.2.1. Molecular clouds: diffuse interstellar bands. The diffuse interstellar absorption bands (DIBs) are ubiquitous absorption features observed in the line of sight to stars that are obscured by diffuse or translucent interstellar clouds. Close to 500 bands have been reported to date in local and extragalactic environments spanning from the near ultraviolet (UV) to the near infrared (IR) (Snow and McCall 2006). Various candidates have been proposed as carriers for the bands, ranging from impurity-doped dust grains, to molecules, to atoms. Today the DIBs are widely thought to be associated with carbon molecules and ions (polycyclic aromatic hydrocarbons (PAHs), carbon chains, fullerenes) that are part of an extended size distribution of interstellar dust (Sarre 2006, Snow and McCall 2006). Astronomers 


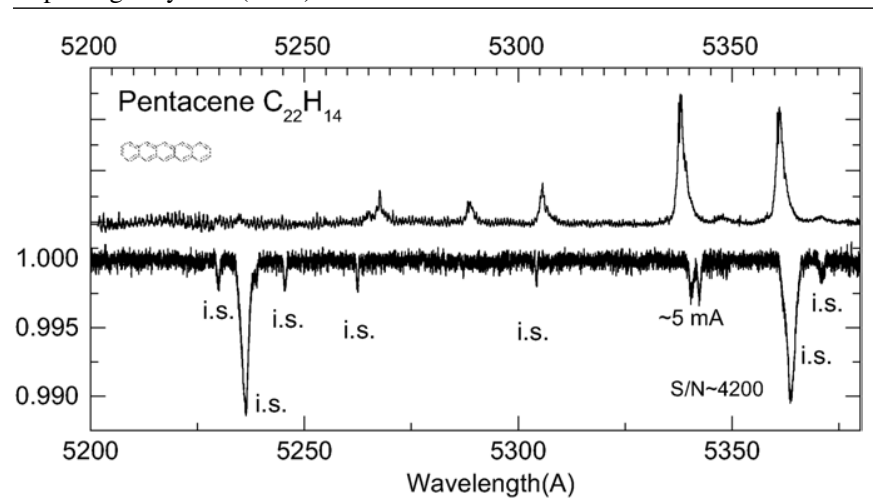

Figure 2. The top trace shows the (inverted) laboratory absorption spectrum (in arbitrary units) of the neutral PAH molecule pentacene $\left(\mathrm{C}_{22} \mathrm{H}_{14}\right)$ prepared in a cold supersonic free jet expansion. The lower trace shows the average absorption spectrum of interstellar translucent clouds (in normalized flux units) providing, for the first time, accurate upper limits for the abundances of interstellar PAHs in the optical. $\mathrm{S} / \mathrm{N}$ refers to signal-to-noise, i.s. to interstellar and $5 \mathrm{~m} \AA$ is the resolution (from Salama et al (2011)).

are very interested in the molecules that carry the DIBs, because these molecules may make up the largest cache of organic material in the universe. Recent advances in laboratory techniques have made it possible to measure the spectra of cold molecules and ions under conditions that are relevant to astrophysics (Salama 2008). As a result, accurate upper limits for the abundances of PAH molecules along the lines of sight of translucent clouds have been reported for the first time (Salama et al (2011), figure 2), while coincidences with naphthalene $\left(\mathrm{C}_{10} \mathrm{H}_{8}^{+}\right)$and anthracene $\left(\mathrm{C}_{14} \mathrm{H}_{10}^{+}\right)$cation bands have been tentatively reported for DIBs in the line-of-sight of Cernis 52 (BD +31 640), an early type reddened star behind the Perseus supernova remnant (SNR) that shows anomalous microwave emission (Iglesias-Groth et al 2008, 2010). A near coincidence between a DIB and a weak absorption feature of the diacetylene cation $\left(\mathrm{C}_{4} \mathrm{H}_{2}^{+}\right)$was also detected in the average spectrum of 11 reddened stars (Krełowski et al 2010), while a coincidence was tentatively reported between a weak DIB observed in the lines of sight of two objects and a band associated with propadienylidene $\left(\mathrm{H}_{2} \mathrm{C}_{3}\right)$ by Maier et al (2011). All coincidences reported to date are tentative and point to hydrocarbon molecules.

\subsubsection{Molecular clouds: molecular anions. Molecular} anions were predicted many years ago to be abundant in the interstellar medium (ISM) (Sarre 1980, Herbst 1981). Subsequent chemical considerations by Terzieva and Herbst (2000) indicated that efficient electron attachment occurs once a carbon chain reaches six atoms. Molecular anions, however, have only recently been detected in space through a combination of spectroscopic laboratory measurements and observations of the molecular envelope of the star IRC+10216 and of the dense Taurus molecular cloud TMC-1 (McCarthy et al 2006). McCarthy et al (2006) showed that the unidentified harmonic sequence found by Kawaguchi et al (1995) in IRC+10216 was $\mathrm{C}_{6} \mathrm{H}^{-}$. The number of detected molecular anions has increased dramatically as a result of laboratory studies since then. These include $\mathrm{C}_{4} \mathrm{H}^{-}$in IRC +10216 by
Cernicharo et al (2007), $\mathrm{C}_{8} \mathrm{H}^{-}$in TMC-1 by Brünken et al (2007) and in IRC+10216 by Remijan et al (2007), $\mathrm{CN}^{-}$in IRC+10216 (Agúndez et al 2010), and $\mathrm{C}_{3} \mathrm{~N}^{-}$in the same object (Thaddeus et al 2008). Sakai et al (2010) detected $\mathrm{C}_{4} \mathrm{H}^{-}, \mathrm{C}_{6} \mathrm{H}^{-}$and $\mathrm{C}_{8} \mathrm{H}^{-}$in a starless core of a molecular cloud (Lupus-1A). This wealth of observational data has renewed interest in the effects of molecular anions on interstellar chemistry (see, e.g., Walsh et al (2009)).

2.2.3. Molecular clouds: polyaromatic hydrocarbons. These emission features, known as the unidentified infrared (UIR) bands, were first discovered by Gillet et al (1973) and attributed to $\sim 10 \AA$ size grains by Sellgren (1984). These UIR bands are now generally attributed to PAHs (see Salama (2008) and references therein). The features of this universal spectrum provide information on the physical conditions in the emitting regions and the nature of the molecular carriers. Puget and Léger (1989) and Allamandola et al (1989) have proposed a model dealing with the UIR interstellar emission features where PAHs are present as a mixture of radicals, ions and neutral species. The ionization states reflect the ionization balance of the medium while the size, composition and structure reflect the energetic and chemical history of the medium. The proposed excitation mechanism of the IR bands is a one-photon mechanism that leads to the transient heating of the PAHs by stellar photons. The IR emission bands are associated with the molecular vibrations of molecular PAH species (discrete bands) and larger carbonaceous grains (continuum-like structures). In this model, PAHs constitute the building blocks of interstellar carbonaceous dust grains and play an important role in mediating energetic and chemical processes in the ISM. However, exploitation of these features as astrophysical probes has been slow in developing because the IR properties of PAHs under interstellar conditions were largely unknown for at least 20 years after the bands were discovered. During the past two decades, advanced experimental and computational laboratory astrophysics programs have been developed to collect data to test and refine the PAH hypothesis. The information for hundreds of PAH molecular species is now compiled in databases that allow astronomers to quantitatively interpret their observations for a variety of environments in our local galaxy and in extragalactic environments (Malloci et al 2007, Bauschlicher et al 2010).

2.2.4. Dark interstellar clouds. The chemistry that occurs in dark interstellar clouds, and especially in the denser regions of such clouds known as cold cores, is an unusual one. Although organic molecules appear to grow in these regions, they are very unsaturated and consist mainly of bare carbon clusters, radicals of the $\mathrm{C}_{n} \mathrm{H}$ and species with two hydrogen atoms, such as $\mathrm{c}-\mathrm{C}_{3} \mathrm{H}_{2}$. This unusual pattern of growth exists despite the fact that molecular hydrogen is the dominant species in the gas and might be expected to hydrogenate the molecular species into more saturated forms. Although the basic mechanism for the growth of unsaturated species in these cold regions was worked out in the early 1970s (Watson 1973, Herbst and Klemperer 1973), the last ten years have 
witnessed some very important laboratory work in rounding out the picture. Before then, it was thought that all organic neutrals are produced via syntheses based entirely on ionmolecule reactions, which synthesize precursor organic ions that do not react with $\mathrm{H}_{2}$, but instead come apart following dissociative recombination reactions with electrons. This picture was incomplete because (a) there was little evidence concerning the actual products of dissociative recombination and (b) the growth of neutral species via reactions involving radicals and regular neutral species was not considered because it was assumed not to occur at low temperatures. Thanks to laboratory astrophysics, the picture has changed. The products of dissociative recombination have now been studied in the laboratory mainly by the use of storage rings in Denmark, Sweden and Germany (Geppert and Larsson 2008, Petrignani et al 2009) in which molecular ions can be cooled down before reaction with electrons. Rapid radical neutral reactions have been studied with Laval nozzles to temperatures down to near $10 \mathrm{~K}$ in laboratories in Rennes, France, and Birmingham, UK (Chastaing et al 2001, Sims 2006). Between these two sets of experiments, our knowledge of the chemical mechanism of molecular growth in cold clouds has become much more complete down to near $10 \mathrm{~K}$.

2.2.5. Pre-stellar cores. Pre-stellar cores have begun the evolutionary journey to form low- and medium-mass stars. They have temperatures of around $10 \mathrm{~K}$ and a gas density of approximately $10^{4} \mathrm{~cm}^{-3}$. At this stage, the collapse is isothermal because any heat developed is radiated away by atoms and molecules. The gaseous cores are dominated by hydrogen, helium and deuterium, as many, if not most, of the heavier molecules are depleted onto dust particles. For example, the abundance of $\mathrm{CO}$ drops precipitously toward the center of pre-stellar cores (Bacmann et al 2002, 2003). The evidence is not as clear cut for other heavy species but their low abundance is determined indirectly by detailed simulations of the deuterium fractionation chemistry, which show a huge fractionation effect in which deuterated isotopologues (e.g. $\mathrm{H}_{2} \mathrm{D}^{+}$) can be very abundant (Roberts et al 2004). Such a large effect can only occur in the near absence of heavy reactive species (Vastel et al 2006). The chemical simulations are based heavily on experimental measurements of rate coefficients involving deuterated species, such as those obtained in an ion trap (Schlemmer et al 2006). The extent of depletion of species such as $\mathrm{CO}$ is confirmed by measurements on the rate of desorption of this species from dust particles, which is not rapid enough to keep a large amount of material in the gas (Öberg et al 2009a).

2.2.6. Hot cores and corinos. Hot cores and corinos are warm objects $(100-300 \mathrm{~K})$ associated with low-mass protostars or young stellar objects of high mass. In these objects, the inventory of gas-phase organic molecules is quite different from what it is in cold interstellar clouds, where the molecules are mainly unsaturated (hydrogen-poor). Instead, in hot cores and corinos the organic molecules are much more terrestriallike and consist of simple alcohols, esters, ethers and nitriles. For many years, it was thought that gas-phase reactions might produce these molecules, but laboratory experiments (Horn et al 2004) show that some of the reactions suggested do not occur or are inefficient. A new school of thought has arisen that the molecules can be produced on the surfaces of dust particles and then desorbed or evaporated into the gas. Several suggestions were made including the production of organic molecules on cold grains, mainly via atomic addition reactions, and the production of these molecules via radicalradical association reactions during the actual heating up of a cold cloud into a hot core because of star formation (Herbst and van Dishoeck 2009). The production of radicals in this latter view comes from photon bombardment of simple surface species such as methanol, produced during the cold era (Garrod and Herbst 2006). Although laboratory experiments have not completely ruled out the idea that more complex species can be produced on cold surfaces, new experiments seem to confirm the radical-radical hypothesis (Öberg et al 2009b).

2.2.7. Protoplanetary disks. Protoplanetary disks are dense objects of gas and dust that rotate around newly formed lowmass stars and may be the precursors of solar-type systems. Astronomers have obtained both rotational and vibrational spectra of molecules in these disks and the molecular inventory is a strong function of how far the molecules lie from the central star and how high they lie off the midplane of the disk. The chemical models used to simulate the chemistry of these complex objects owe much to laboratory astrophysics. One recent success has been an understanding of how some $\mathrm{CO}$ can be in gaseous form at temperatures well below its sublimation point despite the high density of dust particles, which should guarantee that all $\mathrm{CO}$ should be in the form of ice mantles. Recent experiments on the photodesorption of $\mathrm{CO}$ indicate that the efficiency per photon of photodesorption for UV radiation is approximately $10^{-3}$, which under the conditions of protoplanetary disks can explain why $\mathrm{CO}$ can be detected in the gas phase (Öberg et al 2009a, Hersant et al 2009). The recent detection of acetylene $\left(\mathrm{C}_{2} \mathrm{H}_{2}\right)$ and $\mathrm{HCN}$ in hotter regions near the central star can be explained by chemical models that make use of numerous laboratory studies of reactions at temperatures much higher than $300 \mathrm{~K}$ (Agúndez et al 2008a, Harada et al 2010).

2.2.8. Metal hydride spectra of L and Ttype stars. Refractory hydrides such as $\mathrm{FeH}, \mathrm{CrH}, \mathrm{CaH}$ and $\mathrm{MgH}$ have recently been found to be abundant in the atmospheres of M, S and L subdwarf-type stars (Kirkpatrick 2005), as deduced from optical spectroscopy of these objects. In fact, the shift from prominent spectra of metal oxides to metal hydrides is dramatic in the transition from $\mathrm{M}$ type to $\mathrm{L}$ and $\mathrm{T}$ type sub-dwarfs (Burrows et al 2002). These brown sub-dwarfs, especially the $\mathrm{L}$ types, are extremely important for the understanding of planet formation, as they trace the intermediate stage between stars that undergo nucleosynthesis and those that do not, i.e. planets. Hydride spectra such as that of $\mathrm{CrH}$ are also excellent tracers of very cool stellar atmospheres (Burrows et al 2002) and may be an important key in identifying planets. None of this work would have occurred without laboratory spectroscopic measurements, conducted across a broad spectral range (see, 
e.g., Harrison et al (2006)). Laboratory studies of $\mathrm{CrH}$, for example, have been carried out using a variety of spectral techniques, including laser-induced florescence (Chowdhury et al 2005), Fourier transform infrared (FTIR) spectroscopy (Bauschlicher et al 2001) and millimeter/sub-mm direct absorption methods (Halfen and Ziurys 2004). Such hydrides are not stable under terrestrial conditions and must be created by unusual synthetic techniques involving laser ablation, hollow cathode sources and Broida-type ovens. Such work has provided not only wavelengths for spectral identification, but other important physical properties such as electronic state terms, energy levels and Einstein A coefficients, which are essential for astrophysical interpretation of stellar/planetary atmospheres (see, e.g., Burrows et al (2005)). Not all hydrides have been as well characterized as $\mathrm{CrH}$, however, and much lab work needs to be done for species such as $\mathrm{FeH}$ and $\mathrm{TiH}$.

\subsubsection{Comets. Comets offer a unique opportunity to study} organic astrochemistry, knowledge of which till recently has largely been obtained from remote astronomical observations and from laboratory simulations of the formation and evolution of organic molecules in various cosmically relevant environments. Comets are considered as the most primitive objects in the solar system. The composition and the structure of cometary nuclei contain a record of the primordial solar nebula at the time of their formation. Cometary nuclei are made of refractory solids and frozen volatiles. The composition of the volatile component is similar to that observed in dense molecular clouds reflecting the close relationship between cometary materials and interstellar icy grain mantles. Hence, in comets the composition of the volatile ices is largely dominated by $\mathrm{H}_{2} \mathrm{O}$ ice (about $70-90 \%$ ) while other major components include $\mathrm{CO}, \mathrm{CH}_{3} \mathrm{OH}, \mathrm{CO}_{2}$ and $\mathrm{H}_{2} \mathrm{CO}$ (Salama 1998, Bockelée-Morvan et al 2004, Fink 2009).

Comets are also thought to have been a major source for the volatile ices on planetary bodies. Thus, cometary ices constitute a link between interstellar and solar system materials. The captured materials from sample return missions provide new insight into the formation of our solar system. The Stardust mission flew through the near-nucleus coma of comet 81P/Wild 2 on 2 January 2004, swept up material using aerogel collectors and returned these samples to the Earth on 15 January 2006. Stardust is the first space mission to bring back solid material from a known body other than the Moon. One of the key questions that the Stardust samples addressed is the origin of primitive organic matter in the solar system. After the recovery of the Sample Return Capsule, the returned material from Stardust was examined in the laboratory with the goal to determine the nature and amount of the returned samples (Brownlee et al 2006, Hörz et al 2006, Sandford et al 2006, McKeegan et al 2006, Keller et al 2006, Flynn et al 2006, Zolensky et al 2006).

Laboratory astrophysics played a crucial role in the optimization of the knowledge gained from the return of these extraterrestrial samples. An impressive battery of advanced laboratory astrophysics techniques was called upon to help decipher the information contained in the returned samples. The techniques involved transmission electron microscopy (TEM), Raman and FTIR spectroscopy, time of flight secondary ion mass spectrometry (TOF-SIMS) and scanning electron microscopy using energy-dispersive x-ray (SEM-EDX) analyses, among others. These laboratory studies show the highly heterogeneous nature of the collected cometary grains and reveal an interesting distribution of organic material, including the detection of amide, carboxy and alcohol/ether groups (see, e.g., Cody et al (2008) and Clemett et al (2010)) and the amino acid glycine (Elsila et al 2009). While concerns remain as to the organic purity of the aerogel collection medium and the thermal effects associated with hypervelocity capture, the majority of the observed organic species appear indigenous to the impacting particles and are hence of cometary origin. Additionally, though the aromatic fraction of the total organic matter present is believed to be small, it is notable in that it appears to be $\mathrm{N}$ rich. Spectral analyses in combination with instrumental detection sensitivities suggest that $\mathrm{N}$ is incorporated predominantly in the form of aromatic nitriles (R-CN) (Clemett et al 2010).

2.2.10. Exoplanetary atmospheres. In addition to mass and radius, other properties of an exoplanet (e.g. temperature and composition) can be determined using spectral changes during eclipses. Since the first thermal emission from an exoplanet was discovered (Charbonneau et al 2005, Deming et al 2005), a number of other firsts have been reported. One was the discovery of strong evidence for water vapor in the atmosphere of an exoplanet (Tinetti et al 2007). Signatures of water and carbon dioxide are now observed both in absorption and emission in a number of exoplanet atmospheres (Knutson et al 2008, Charbonneau et al 2008, Grillmair et al 2008). The measurement of temperature differences between the nightside and day-side of a tidally locked close-in hot Jupiter has emphasized the role of stellar radiation on the planetary atmosphere (Knutson et al 2007). While the composition of exoplanets at or above Jupiter in mass is not in doubt (they must be gas giants composed of hydrogen and helium), spectroscopy is needed to determine the composition of the smallest planets discovered to date (the so-called 'super-Earths'), since they may also be rocky (like the Earth) or icy. Near IR spectroscopy of one such super-Earth has ruled out hydrogen gas, unless there are thick clouds (Bean et al 2010). These observations are also consistent with the presence of hot water vapor (steam), in which case the planet might have an icy rather than rocky core. All these discoveries rely heavily on spectroscopic modeling of the stellar atmosphere (Hauschildt et al 2009), as well as the exoplanet atmosphere (Seager et al 2005, Miller-Ricci et al 2009, Kaltenegger and Sasselov 2010) and thus on the supporting laboratory astrophysics and atomic and molecular line data (Castelli and Kurucz 2004, Rothman et al 2005).

\subsection{Condensed matter physics}

2.3.1. Outer solar system ice. The connection between the ISM and solar systems profoundly influences our understanding of the birth and death cycles of stars in our Galaxy. Present models of star formation suppose that interstellar amorphous ice grains accreted to form the outer 
rim of the solar system from Oort cloud to Kuiper belt objects (KBOs) (Jewitt 1999). Based on these models, outer solar system icy bodies with surface temperatures $<100 \mathrm{~K}$ form amorphous ices. At these temperatures amorphous ices remain stable over the lifetime of our star $\left(4.5 \times 10^{9} \mathrm{yr}\right)$. Galilean icy satellites like Europa at $5 \mathrm{AU}$ with surface temperatures $\sim 120 \mathrm{~K}$ are crystalline. Beyond Jupiter, the rest of the outer solar system icy bodies have equilibrium surface temperatures $<100 \mathrm{~K}$ and hence are expected to contain amorphous ices. These are Saturnian icy moons and rings at $10 \mathrm{AU}(\sim 100 \mathrm{~K})$, Uranian satellites around $20 \mathrm{AU}$, trans Uranian objects and KBOs $(\sim 50 \mathrm{~K})$ at $40 \mathrm{AU}$ from the Sun and the Oort cloud $(\sim 30 \mathrm{~K})$ spanning up to several thousands of AU towards the local ISM.

Near IR spectroscopic studies carried out in the laboratory (Grundy and Schmitt 1998) revealed that amorphous ices show significantly different absorption features in this region compared with the crystalline ices, as shown in the lower part of figure 3. Recent spectrally resolved observations showed that the surface ices of trans Uranian icy bodies (Grundy et al 2006), trans Neptunian objects (TNOs) (Trujillo et al 2007, DeMeo et al 2010) and KBOs (Jewitt and Luu 2004) are significantly crystalline, based on comparison of these spectra with the laboratory data. Some recent models attribute the surface crystallinity to micrometeorite impacts (Porter et al 2010). This counter-intuitive observation, supported by laboratory data, has opened up a new chapter in our understanding of the evolution of icy bodies in the solar system and in the ISM.

Recently it has also been shown in the laboratory (Zheng et al 2009) that the crystallinity of ice at $>50 \mathrm{~K}$ is not destroyed or altered to amorphous-like form by electron irradiation under conditions similar to those that exist on KBOs and comets originating from them. However, it is still unclear how the amorphous ice grains in the ISM are converted into the crystalline surface layer of KBOs and whether the subsurface of KBOs is amorphous or crystalline and hence the comets originating from them. More laboratory studies are needed in order to resolve this amorphous-crystalline puzzle that connects the ISM with the outer solar system.

2.3.2. Cometary ice, chemistry and the origins of life? One of the working postulates of the origins of life is that cometary impacts brought organic chemicals and water to the Earth (Whittet 1997, McClendon 1999, Matthews and Minard 2006). Comets are expected to retain the interstellar amorphous ice structure. Organic rich comets have been found to be highly porous (Richardson et al 2007, A'Hearn 2008). One of the outstanding questions is whether the delicate building blocks of life survived the comet impacts on Earth. With a very similar ice grain composition between comets (Crovisier et al 2004) and interstellar ice grains (Gibb et al 2004), these ices are dominated by $\mathrm{H}_{2} \mathrm{O}$, followed by $\mathrm{CO}_{2}, \mathrm{CO}$, methanol $\left(\mathrm{CH}_{3} \mathrm{OH}\right)$, hydrocarbons, nitrogen-containing molecules $\left(\mathrm{NH}_{3}\right.$ and derivatives) and sulfur-containing molecules such as OCS, as well as minerals such as silicates. All these ingredients $(\mathrm{H}, \mathrm{C}, \mathrm{N}, \mathrm{O}, \mathrm{S}$ and minerals containing these elements) are essential for all forms of life on Earth as is also phosphorus (P), which is yet to be positively detected in comets. Laboratory studies using the primitive molecules mentioned above and simulating the composition of comet and interstellar ice grains have shown that radiation processing of these ices indeed produced building blocks of life upon subsequent heating to evaporate ice (Dworkin et al 2001, Bernstein et al 2002, Deamer et al 2002, Muñoz Caro et al 2002, Elsila et al 2007, Nuevo et al 2009). These laboratory studies are critical, corroborating one of the possible origins of life on Earth.

Recent laboratory studies have also enhanced our understanding of the mechanisms involved in the radiation processing of organic molecules in ices that result in the formation of complex building blocks of life (Gudipati 2004, Gudipati and Allamandola 2004, 2006, Bouwman et al 2010). Using PAHs as probes embedded in ices, these laboratory studies have shown that radiation induced ionization of PAHs is an important first step, forming electron and $\mathrm{PAH}$ radical cation pairs in ice, which subsequently lead to the formation of oxidized PAHs. These laboratory studies have opened up a new understanding of chemistry in ices, involving charged species, bringing us one step closer to understanding how ices evolve under irradiation. Charged ice grains behave differently compared with their neutral counterparts due to strong longrange Coulomb forces. The implications of these studies to astrophysics and planetary sciences are slowly unfolding (Kalvans and Shmeld 2010).

\subsection{Plasma physics}

2.4.1. Accretion disks and magnetorotational instability. Accretion disks form in various astrophysical systems including young stars, protostars and some CVs. The accretion disk forms because the accreting matter brings substantial angular momentum, which must be transported away in order for the matter to move inward. Physical viscosity is far too small and it is generally believed that magnetohydrodynamic (MHD) turbulence is responsible for the angular momentum transport. At present, the leading candidate to drive such turbulence is magnetorotational instability (MRI) (Balbus and Hawley 1991, 1998), with the turbulence itself produced by secondary instabilities that convert the structures generated by the MRI into multiscale turbulent fluctuations (Pessah 2010, Pessah and Goodman 2009). A major challenge in coming to understand the MRI comes from the limitations of various approaches. Analytic and semi-analytic theories have made great progress (Julien and Knobloch 2010) but always struggle to define turbulent states. The astrophysical systems have very large $R e$ and $R m$, where $R e$ is the usual viscous Reynolds number and $R m$ is the analogous magnetic Reynolds number, characterizing how slowly the magnetic structures are dissipated by resistive heating of the plasma. Numerical simulations cannot reach the astrophysical regime, being very limited in $R e$ and having values of $R m$ that can be larger but remain limited. The past decade has seen laboratory experiments that reported observation of the MRI (Stefani et al 2006) and a helical variant (Sisan et al 2004). These experiments complement the simulations, having larger values of $R e$ than the simulations can produce but smaller values of $R m$. Experiments to date have been performed with a liquid 
metal conducting fluid, a system well described by MHD theory. The combination of experiments, simulations and observations now provides a more complete set of information for theoretical work that seeks to identify the important scaling parameters and to provide a unified understanding of MRI across all regimes.

\subsubsection{Young stellar objects: jet structure. Many open} questions remain in the study of jets emanating from young stellar objects (YSOs) (Reipurth and Bally 2001). These nonrelativistic beams of hypersonic plasma are likely magnetized and are known to cool effectively via radiation losses. Of particular interest for astrophysics are issues related to the internal jet structure. Are the hypersonic beams of plasma (hyperfast mode in the case of MHD jets) structurally smooth or inherently inhomogeneous?

Depending on the stability conditions of the jets this question speaks directly to the launch mechanisms of the jets as structurally smooth jets, implying time independent conditions at the central engine launching the jet. Recent observations using Hubble Space Telescope and other highresolution platforms indicate that jets may contain significant sub-radial structure $\left(\delta x<r_{\text {jet }}\right.$ ), which implies that jets may be inherently heterogeneous or 'clumpy' phenomena (Hartigan and Morse 2007, Hartigan et al 2011).

Recent experimental studies have attempted to explore this issue by developing platforms that can create steady jet beams as a starting point for further work. Of particular note have been the pulsed power studies of Lebedev et al (2002) who were able to develop stable hypersonic radiative jets. These jets have high Mach numbers $(M \sim 20)$ and have been shown to propagate without disruption over long distances, achieving aspect ratios of 10 or more. Shorter duration jets have also been produced in a number of studies (Foster et al 2005). In some cases these experimental platforms have allowed researchers to explore the interaction of jets with large-scale obstacles (Hartigan et al 2009). This is an astrophysically relevant issue as jets from young stars are observed, in some cases, to be deflected by clumps or clouds in their path. Deflection of jets by winds induced by the motion of the jet source through a background has also been observed and this process has been studied in the laboratory as well (Lebedev et al 2004).

Thus experimental studies to date have shown that stable hypersonic jets can propagate over long distances and that even when interacting with side winds the jets are not fully disrupted. Future studies should focus on the generation and propagation of 'clumps' within the beams.

\subsubsection{Young stellar objects: magnetized jets. Astrophysical} jets are believed to form via a combination of accretion, rotation and magnetic fields (Pudritz et al 2007). The central engine may be a star, a compact object like a black hole or surrounding accretion disk. YSO jets are also believed to form via magnetized accretion disks and many open issues remain concerning both the magneto-centrifugal launch processes and the propagation of the magnetized jet at large distances from the central engine. In general theorists expect the fields to be strong to moderate as characterized by the plasma beta which is the ratio of gas (g) to magnetic (B) pressures $\beta=P_{\mathrm{g}} / P_{\mathrm{B}} \leqslant 1$.

Using a planar magnetized coaxial plasma gun, Bellan (2005) and Bellan et al (2005) have developed a platform to study MHD jet launching. The premise behind the experiments is that the basic magnetic dynamics near a star-disk system, namely the winding up of poloidal field lines generated by the central disk + star rotation, can be simulated in the laboratory by applying a voltage across coaxial electrodes in the presence of a background colloidal field. The magnetic helicity injection with these boundary conditions leads naturally to collimated unstable plasmas whose dynamics may be indicative of diskdriven jets and plasmoids.

A second approach to the study of magnetized YSO jets comes from experiments using radial plasma sources, which consist of a pair of concentric electrodes connected radially by thin metallic wires or a thin foil (Lebedev et al 2005, Ciardi et al 2009). Resistive heating of the wires or foil produces a plasma. If wires are used, when they break, toroidal flux from below drives a magnetic bubble $(\beta<1)$ and a collimated jet forms on the axis. The jet goes unstable due to kink modes and evolves into a series of hypersonic clumps. When a foil disk is used, the process becomes episodic with a series of magnetic bubbles and jets forming one after the other.

Laboratory studies of magnetized jets relevant to YSOs have offered a new window into the three-dimensional (3D) dynamics of magnetized plasma systems. Helicity injection and kink mode instabilities have been followed in ways that already demonstrate new pathways of jet evolution not previously considered in analytic or computational studies.

2.4.4. Young stellar objects: radiative jets. Along with magnetic fields, radiative cooling is another important process occurring in YSO jets. In this context radiative cooling means that optically thin emission from shock excited atoms and ions will carry away a significant amount of energy from the system. Systems are radiatively cooling when the timescale for energy loss $\left(t_{\text {cool }}=e / \dot{e}\right)$ is less than the characteristic hydrodynamic timescale $\left(t_{\mathrm{h}}=L / c\right)$ where $e, \dot{e}, L$ and $c$ are thermal energy, thermal energy loss rate, system scale and speed of sound, respectively. As has been shown in numerous studies, radiative cooling will produce dramatic differences in the evolution of jet systems compared with adiabatic flows (Blondin et al 1990). In particular, the collapse of bow shocks onto the jet body will occur when thermal pressure generated at the shock is removed via the radiative cooling. Resolution issues hamper numerical simulations of jet dynamics with radiative cooling. A detailed understanding of instabilities at cooling bow shocks, for example, has not yet been achieved.

Experiments have produced radiative jets by creating radially imploding plasmas having an axial velocity component. In early work lasers were used to irradiate conically shaped targets (Farley et al 1999). Work using wire arrays (Lebedev et al 2002) created stagnation of plasma flow on the axis of symmetry, forming a standing conical shock effectively collimating the flow in the axial direction. This scenario is essentially similar to that discussed by Canto et al (1988) as a purely hydrodynamic mechanism for jet formation 
in astrophysical systems. In both types of experiments, the diameter of the jet decreased with increasing atomic number, providing direct evidence of radiative cooling. In a more recent experiment, a ring-shaped laser spot was employed to produce an imploding $\mathrm{Cu}$ plasma, generating a jet that penetrated into adjacent gas (Tikhonchuk et al 2008). Analysis showed the experimental parameters to be rigorously well scaled to astrophysical cases. Structure was seen in the shocked ambient medium, providing evidence relevant to the instabilities at cooling bow shocks.

Thus experiments have produced radiative hypersonic jets in laboratory settings, allowing existing theories of jet dynamics to be explored and opening up new domains of investigation beyond the reach of existing analytic methods and simulations.

\subsubsection{Hydrodynamic stability of protoplanetary accretion} disks. It is widely accepted that MRI plays an important role in generating turbulence that transports angular momentum outward in accretion disks (Balbus and Hawley 1998). The electrical conductivity of portions of protoplanetary disks is thought to be so low, however, that the magnetic field is not well coupled and that MRI cannot operate. It was proposed that hydrodynamic Keplerian flow can be unstable to finite amplitude perturbations and that this could lead to angular momentum transport. Recent laboratory experiments of hydrodynamic Keplerian flow between two cylinders have found no evidence of such an instability, up to Reynolds numbers of $2 \times 10^{6}$ (Ji et al 2006, Schartman et al 2011). This negative result weighs against instability of Keplerian flow as an angular momentum transport mechanism in accretion disks and encourages us to look for other mechanisms.

2.4.6. Equation of state for planetary interiors. Present-day observations of planets can determine only their mass, size and perhaps surface composition. One wants to know much more such as the structure of the planet, the properties of the interior matter and whether gas-giant planets required an ice and rock core to nucleate their formation. One seeks a self-consistent model in which the local density at some radius is determined by the materials present and the local pressure, while the integrated density profile within the observed planetary radius corresponds to the mass of the planet (see the recent review by Fortney and Nettelmann (2010)).

The relations between density, pressure and other thermodynamic quantities are the equations of state (EOS). For the specific case of Jupiter, Saumon and Guillot (2004) have shown that the uncertainties in the EOS are the dominant limitation to understanding the structure of the planet. Laboratory measurements are essential to advance this field; the relevant EOS theory is difficult, both intrinsically and with regard to choosing appropriate assumptions. The first two first-principles models, using very similar methods, implied different amounts and distributions of heavy elements in Jupiter (Fortney et al 2009). Laboratory data have been used to adjust other EOS models (Fortney and Nettelmann 2010) and researchers are actively acquiring more data (Eggert et al 2008, Hicks et al 2009).
Figure 4 shows a comparison of state-of-the-art data and theory for the pressure and density produced by a strong shock wave in cryogenic He after compression to some initial density (adapted from Fortney et al (2009)). The theory curves were produced by first-principles calculations using a combination of path-integral-Monte-Carlo and density-functional-theory, molecular-dynamics calculations (Militzer 2009). The data points were inferred from direct measurements of shock velocity in $\mathrm{He}$ and in quartz, using standard techniques (Eggert et al 2008). There is reasonable agreement between data and theory for high pre-compressions, but not for low precompressions. This indicates that more work is needed to fully understand the compression of He.

Further advances are needed in order to obtain a fully validated account of the properties of $\mathrm{He}$ at relevant densities and pressures. Progress in these areas will complement improved measurements of the abundance of oxygen and of the detailed gravitational field structure by the Juno orbiter (Bolton 2006). Other space missions will identify hundreds of additional Neptune-like to Jupiter-like planets. EOS research during the last decade has provided data that constrain planetary models and demonstrated methods that will produce further data going forward.

\section{Stars and stellar evolution}

Stars and stellar evolution covers 'the Sun as a star, stellar astrophysics, the structure and evolution of single and multiple stars, compact objects, supernovae, gamma-ray bursts, solar neutrinos and extreme physics on stellar scales' (Blandford et al 2010a).

\subsection{Atomic physics}

3.1.1. Solar and stellar abundances of rare earth elements. Accurate heavy-element abundances have recently been determined for the rare earth elements in the Sun and in old, metal-poor Galactic halo stars. These abundances provide insight into the nature of the earliest stellar generations and element formation in the Galaxy. The updated values are the result of extensive new laboratory data for atomic transition probabilities. Data have been published for numerous spectra including La II (Lawler et al 2001a), Ce II (Palmeri et al 2000, Lawler et al 2009), Pr II (Ivarsson et al 2001), Nd II (den Hartog et al 2003), Sm II (Xu et al 2003, Lawler et al 2007), Eu I, II and III (den Hartog et al 2002, Lawler et al 2001c), Gd II (den Hartog et al 2006), Tb II (den Hartog et al 2001, Lawler et al 2001b), Dy I and II (Curry et al 1997, Wickliffe et al 2000), Ho I and II (den Hartog et al 1999, Lawler et al 2004), Er II (Lawler et al 2008a,2008b), Tm I and II (Anderson et al 1996, Wickliffe and Lawler 1997), Lu I, II, and III (den Hartog et al 1998, Quinet et al 1999, Fedchak et al 2000), Hf II (Lawler et al 2007), Os I and Ir I (Ivarsson et al 2003), Pt I (den Hartog et al 2005), Th II and III (Biémont et al 2002, Nilsson et al 2002b) and U II (Lundberg et al 2001, Nilsson et al 2002a).

These new transition probabilities have culminated in more precise solar and stellar abundances of Pr, Dy, Tm, $\mathrm{Yb}$ and $\mathrm{Lu}$ (Sneden et al 2009). As a result, it is now 


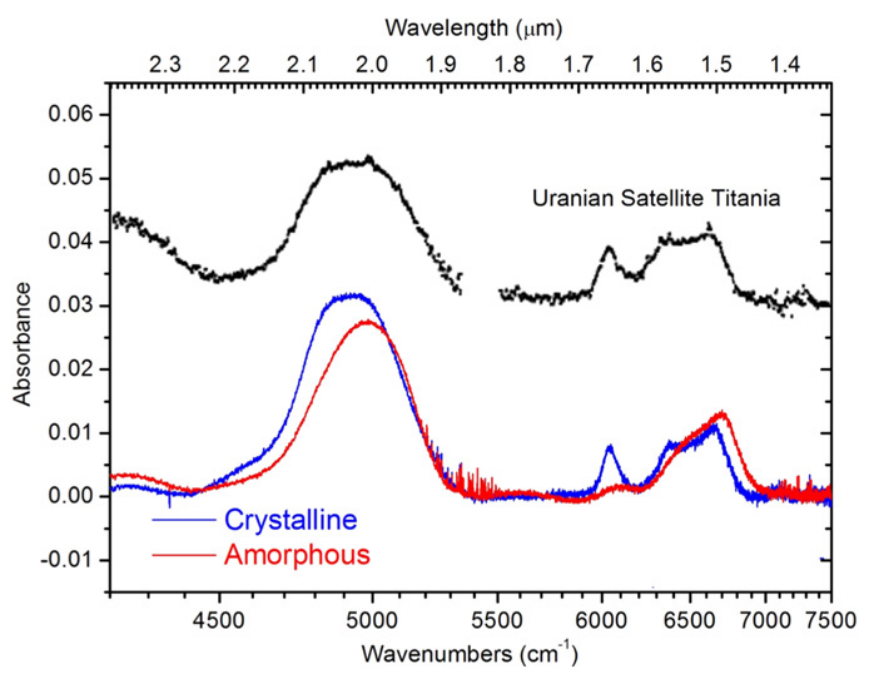

Figure 3. Observed near IR (black) of the Uranian satellite Titania (Grundy et al 2006), compared with the laboratory spectra of amorphous (red) and crystalline (blue) water ice at $10 \mathrm{~K}$ (courtesy of M Gudipati, private communication, and in excellent agreement with the published results of Grundy and Schmitt (1998) and Mastrapa and Brown (2006)). The absorption around $6000 \mathrm{~cm}^{-1}$ $(1.65 \mu \mathrm{m})$ is the most prominent feature of the crystalline ice that is not present in amorphous ices. Other subtle spectral differences can also be seen in the laboratory spectra.

conclusively demonstrated that the abundance pattern for the heaviest elements in the oldest metal-poor halo stars is consistent with the relative solar system abundances for rapid neutron capture (r-process) only elements. This indicates that the r-process that operated in the early Galaxy, soon after the first stars formed, must share some common features withand perhaps is identical to - the r-process that operates now. Thus, the star-to-star relative abundances of these elements should be the same and also consistent with the solar system values. This can be seen in figure 5 where the abundances of three metal-poor halo stars (CS 22892-052, BD +17 3248 and HD 115444) are compared with meteoritic and solar system r-process abundances (den Hartog et al 2006, Sneden et al 2008). Additional elements have been measured since the publication of that figure and the abundance analyses have now been extended to more stars (see, e.g., Sneden et al (2009)).

\subsubsection{The solar abundance problem. Three-dimensional,} time-dependent, hydrodynamical solar atmosphere models are a remarkable computational achievement of the past decade. These models require significantly lower abundances of $\mathrm{C}, \mathrm{N}, \mathrm{O}$ and $\mathrm{Ne}$ to match photospheric spectra (Asplund et al 2004), compared with previous results based on one-dimensional, static non-local-thermodynamicequilibrium (non-LTE) models (see, e.g., Vernazza et al (1976)). However, the new abundances do not agree with helioseismology observations (Bahcall et al 2005a). Christensen-Dalsgaard et al (2009) and others have suggested that increased opacity could bring the helioseismology models back into agreement with observations, but that would require about a $30 \%$ increase in atomic abundances at the base of the convection zone and a few percent in the solar core.

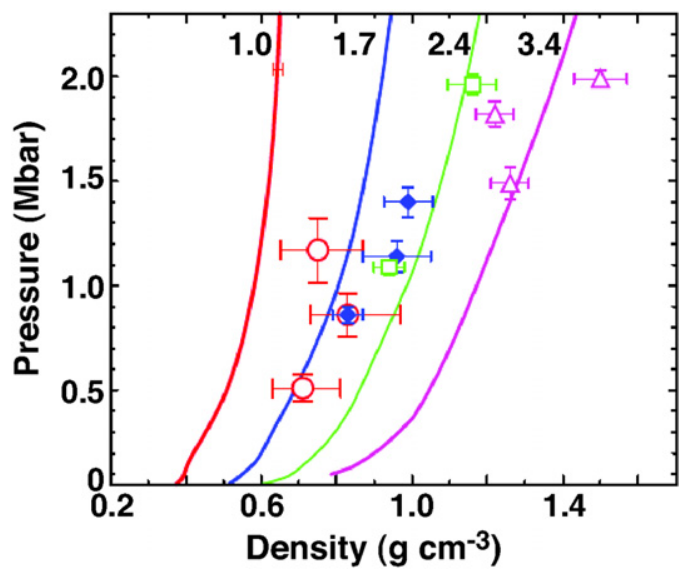

Figure 4. Equation of state data and theory for He. The He is first precompressed, in a diamond-anvil cell, by the factor that labels each curve and then is shocked to high pressure, which allows one to access densities and pressures relevant to gas-giant planets. The density of the He before precompression is the zero-pressure density of cryogenic $\mathrm{He}\left(0.123 \mathrm{~g} \mathrm{~cm}^{-3}\right)$. This figure, adapted from Fortney et al (2009), shows experimental data from Eggert et al (2008) and first-principles theory from Militzer (2009).

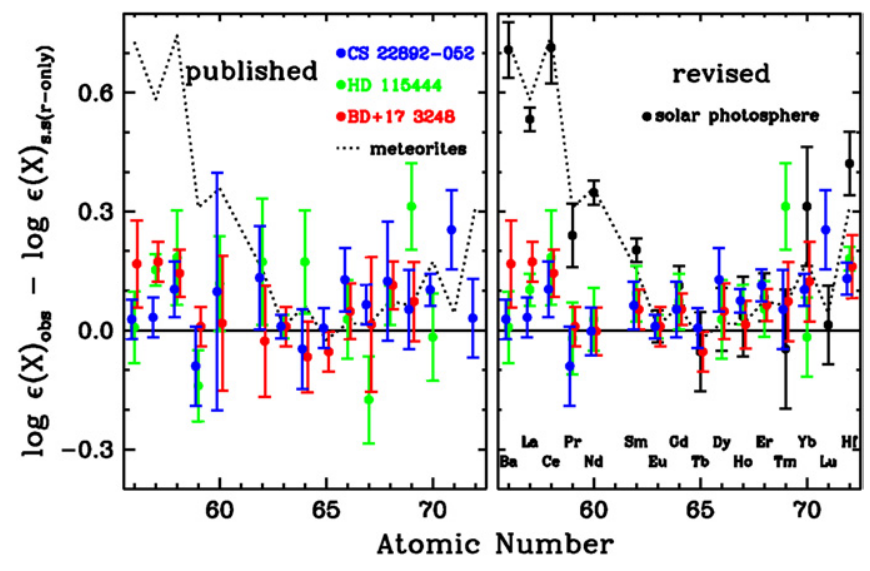

Figure 5. The observed abundances $\epsilon(X)_{\text {obs }}$ relative to the solar system r-process only abundances $\epsilon(X)_{\text {s.s.(r-only) }}$ as a function of atomic number for element $X$. The left panel results are based upon older published atomic data. A large scatter is readily apparent from star-to-star and with respect to the dashed horizontal line, representing the solar system r-process only line (normalized to the element $\mathrm{Eu}$ ). The right panel shows the relative abundances with the newly determined laboratory data (labeled 'revised'). For those elements discussed in section 3.1.1 with new atomic data, the star-to-star scatter has largely disappeared and the new abundances are also consistent with the solar r-process only abundances. Adapted from den Hartog et al (2006) and Sneden et al (2008).

The convergence of various opacity calculations over the past decade (including large contributions from atomic theory and experiment) is a considerable success and thus an opacity increase as large as $30 \%$ may not be reasonable (ChristensenDalsgaard et al 2009). Furthermore, new Z-pinch experimental tests of the iron opacity, under conditions approximating the base of the solar convection zone, show good agreement with the most recent and advanced opacity models (see section 3.4.4; Bailey et al (2007) and Mancini et al (2009)). Additional experiments are needed to test the opacity models under other relevant physical conditions (see Bailey et al 
(2009)). For now, opacities alone do not appear to resolve all the problems fitting helioseismology data and the solution may well lie elsewhere, such as in the EOS (Lin et al 2007, Basu 2010).

3.1.3. The solar corona. Despite decades of research, we still do not understand how the temperature of the solar atmosphere rises from $\sim 6000 \mathrm{~K}$ at the photosphere to more than $10^{6} \mathrm{~K}$ in the corona. Fe XVII is an important system for studying the corona, producing some of the strongest lines seen. It is formed near the peak temperatures of active regions and emits a number of useful diagnostic line ratios for temperature, density and opacity. Resonant line scattering in the strongest solar coronal $\mathrm{x}$-ray line (Fe XVII $3 \mathrm{~d}^{1} \mathrm{P}_{1}$ to ground ${ }^{1} \mathrm{~S}_{0}$, known as $3 \mathrm{C}$ ) has long been thought to contribute to its observed weakness relative to the nearby Fe XVII $3 d^{3} D_{1}$ to ${ }^{1} S_{0}$ line (known as $3 D$ ). Even at a relatively low optical depth, resonant line scattering could in principle also account for morphological effects in images of loop structures (Wood and Raymond 2000). If this were the case, efforts to increase spatial resolution of solar coronal imaging instruments to $\sim 0.1$ arcsec might not be worthwhile. Theoretical calculations of the 3C/3D line ratio have until recently been significantly larger than any of the solar observations. Over the past decade a number of experimental measurements (Brown et al 1998, Laming et al 2000, Brown et al 2001, Gillaspy et al 2011) and ongoing theoretical work (see, e.g., Doron and Behar (2002), Loch et al (2006) and Chen (2008)) have produced convergence on the appropriate line ratio for comparison with observations. With the Fe XVII 3C/3D line ratios on solid ground, Brickhouse and Schmelz (2006) showed that the solar X-ray corona is optically thin in Fe XVII $3 \mathrm{C}$ and, by extension, in all the coronal lines. The blurring seen in some images (e.g., Fe xv from the Transition Region and Coronal Explorer (TRACE) satellite) is thus the result of unresolved spatial structure near the peak temperature. Efforts to observe the solar corona at still higher spatial resolution are thus warranted.

3.1.4. O star winds. Advances in our understanding of the elemental evolution of the cosmos has come about from spectroscopic observations of $\mathrm{O}$ stars carried out using Chandra and the X-ray Multi-Mirror Mission-Newton (XMMNewton) coupled with new laboratory astrophysics data. The powerful radiatively driven winds of $\mathrm{O}$ stars are important sources of chemical enrichment in the universe. Recent analyses of UV P Cygni profiles and $\mathrm{x}$-ray emission line profiles have been used to determine mass loss rates (Fullerton et al 2006, Cohen et al 2010). These studies used the best available wavelengths (accurate to a few $\mathrm{m} \AA$ ) and a relatively complete database of important x-ray emission lines coupled with data on relative line strengths in coronal plasmas, in order to accurately account for blended complexes of Doppler broadened emission lines. The mass loss rate from $\mathrm{O}$ stars was found to be a factor of 3-6 less than previously thought (Cohen et al 2010), a result deriving from recent improvements in atomic data from laboratory and theoretical calculations. This changes our understanding of chemical enrichment of galaxies, especially during their early starburst phase.
3.1.5. Type Ia supernovae. Type Ia supernovae $(\mathrm{SNe})$ are used as standard candles to study dark energy and the expansion of the universe. Chandra and XMM-Newton X-ray observations of young supernova remnants (SNRs) have deepened our understanding of these standard candles. X-ray observations of young SNRs in the Milky Way and the Magellanic Clouds offer a detailed view of Type Ia supernova ( $\mathrm{SN}$ ) ejecta and provide invaluable constraints on the physics of these explosions and the identity of their progenitor systems. Utilizing public domain atomic data, it is now possible to model this $\mathrm{x}$-ray emission and distinguish SNRs resulting from bright and dim Type Ia SNe. This technique has been validated by the detection and spectroscopy of SN light echoes for the Tycho SNR (Badenes et al 2006, Krause et al 2008) and SNR 050967.5 in the Large Magellanic Cloud (LMC) (Badenes et al 2008a, Rest et al 2008). A key advantage of these X-ray studies of nearby SNRs over optical studies of extragalactic SNe is that the SNRs are close enough to examine the circumstellar medium sculpted by the progenitor systems (e.g., the Kepler SNR, Reynolds et al (2007)) and also to study the resolved stellar populations associated with them (Badenes et al 2009). Recent X-ray spectroscopic observations have also discovered emission from $\mathrm{Mn}$ and $\mathrm{Cr}$ in young Type Ia SNRs which can be used to measure the metallicity of the progenitor system (Badenes et al 2008b), one of the key variables that might affect the cosmological use of Type Ia SNe and which cannot be determined for extragalactic SNe.

\subsection{Molecular physics}

3.2.1. Evolved star envelopes: characterizing gas and dust chemistry. Mass loss from evolved stars (asymptotic giant branch (AGB), red giants and supergiants) contributes about $85 \%$ of the material to the ISM (Dorschner and Henning 1995). Such mass loss creates large envelopes of dust and gas surrounding the central star, extending to $\sim 1000$ stellar radii. Establishing the chemical content of stellar envelopes is important in evaluating the overall composition of the ISM. These envelopes can either be oxygen-rich $(\mathrm{O}>\mathrm{C})$ or carbonrich $(\mathrm{C}>\mathrm{O})$. Such shells also have large temperature and density gradients (see, e.g., Ziurys (2006), Kim et al (2010), Maercker et al (2008), Patel et al (2011), Tenenbaum et al (2010a), Polehampton et al (2010) and Schoier et al (2011)). Close to the stellar photosphere, chemical species, as well as dust condensates, form under thermodynamic equilibrium. As the material flows from the photosphere, abundances become 'frozen-out', but then are altered by photochemistry at the shell edge (see, e.g., Cordiner and Millar (2009)). Circumstellar envelopes are consequently unusual chemical factories. The C-rich shell of the AGB star IRC + 10216, for example, has been found to contain over 70 different chemical compounds (Ziurys 2006, Agúndez et al 2008b, Tenenbaum et al 2010a, 2010b). Oxygen-rich stars also have complex chemistries, as observations of the envelope of VY Canis Majoris have demonstrated (Tenenbaum et al 2010a, 2010b). The chemical richness of circumstellar envelopes is illustrated in figure 6. Here composite spectra of the envelopes of IRC + 01216 and VY Canis Majoris are shown at $1 \mathrm{~mm}$ in wavelength. 


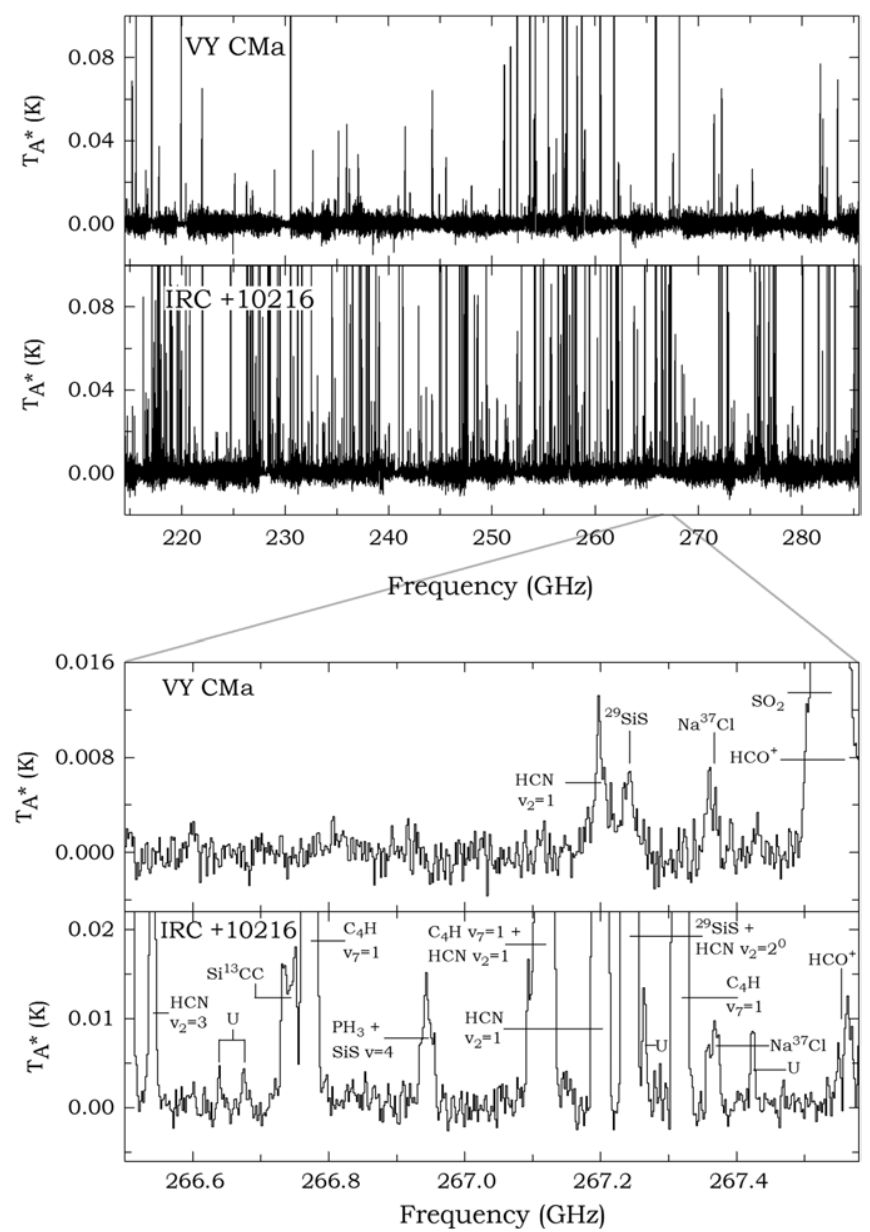

Figure 6. The composite spectra of VY CMa and IRC +10216 across the entire $214.5-285.5 \mathrm{GHz}$ frequency region, showing the rich chemical complexity of both sources (Tenenbaum et al 2010a). The intensity scale is the same for both sources and has been truncated to show the weaker lines. The inset panel displays a select $1 \mathrm{GHz}$ section, centered at $267 \mathrm{GHz}$. The inset panel highlights some of the molecular identifications such as $\mathrm{HCN} v_{2}=1^{1 d}(J=3 \rightarrow 2)$, ${ }^{29} \mathrm{SiS}(J=15 \rightarrow 14), \mathrm{Na}^{37} \mathrm{Cl}(J=21 \rightarrow 20)$ and $\mathrm{HCO}^{+}$ $(J=3 \rightarrow 2)$. The IRC+10216 spectrum at $267 \mathrm{GHz}$ shows a tentative line of $\mathrm{PH}_{3}$ and various other vibrationally excited $\mathrm{HCN}$ features. Without laboratory spectroscopic studies, not a single line in these spectra could be securely identified.

Most circumstellar species have been detected on the basis of their pure rotational spectra, which occur at millimeter and sub-mm wavelengths. IR spectra have also been important for species with no dipole moments, such as $\mathrm{HCCH}$. The input of laboratory spectroscopy, which supplies the critical 'rest frequencies' for line identification, has been crucial in this regard. Recent examples include the identification of negative ions, such as $\mathrm{C}_{4} \mathrm{H}^{-}$and $\mathrm{C}_{3} \mathrm{~N}^{-}$(Thaddeus et al 2008) and $\mathrm{KCN}$ (Pulliam et al 2010).

\subsubsection{Evolved star envelopes: refractory-element-bearing} species. Condensation models predict that dust in circumstellar shells take on a variety of forms, depending on whether the environment is oxygen or carbon-rich (Lodders and Fegley 1999). Almost all the refractory elements (Si, P and metals) are predicted to be in some sort of mineral grain.
In $\mathrm{C}$-rich shells, silicon is contained primarily in $\mathrm{SiC}$, but in $\mathrm{O}$-rich objects in oxide condensates. Phosphorus is thought to be in the form of schreibersite, $(\mathrm{Fe}, \mathrm{Ni})_{3} \mathrm{P}$. Magnesium is contained in silicon and aluminum oxides in O-rich shells and primarily in $\mathrm{MgS}$ in C-rich shells.

Refractory elements in circumstellar environments are not all contained in dust grains, however, as millimeter observations have clearly shown. Nine molecules have been found in circumstellar shells that contain silicon and eleven that contain metals, in the chemist's sense. In C-rich envelopes, the metals are either found in halides $(\mathrm{NaCl}, \mathrm{KCl}, \mathrm{AlF}$ and $\mathrm{AlCl}$ ) or metal cyanides $(\mathrm{MgCN}, \mathrm{AlNC}, \mathrm{MgNC}, \mathrm{KCN}$ and $\mathrm{NaCN}$; Pulliam et al (2010)); in oxygen-rich shells, oxides and hydroxides such as $\mathrm{AlO}$ and $\mathrm{AlOH}$ dominate (Tenenbaum and Ziurys 2010). Aluminum is thought to be condensed into $\mathrm{Al}_{2} \mathrm{O}_{3}$ in O-rich stars (Lodders 2003). The presence of $\mathrm{AlO}$ and $\mathrm{AlOH}$ indicates that photospheric shocks are likely disrupting grains.

In addition, phosphorus-containing molecules appear to be prevalent in circumstellar shells, as evidenced by the recent discoveries of CCP, PN, HCP, $\mathrm{PO}$ and, tentatively, $\mathrm{PH}_{3}$ (see, e.g., Agúndez et al (2007), Tenenbaum et al (2007), Milam et al (2008) and Tenenbaum and Ziurys (2008)). Phosphorus is an important biogenic element and has consequences for the origin of life. Until very recently, few molecules containing this relatively rare element have been observed in the ISM.

Gas-phase, high-resolution, laboratory spectroscopy has been absolutely crucial in establishing the presence of metal and phosphorus-bearing species in circumstellar gas. Recent discoveries, such as $\mathrm{CCP}$ or $\mathrm{AlOH}$, have relied on such work, in particular those employing millimeter direct absorption and Fourier transform microwave methods (see, e.g., Apponi et al (1993) and Halfen et al (2009)). Many potential species of this type are highly reactive, and require unusual, non-equilibrium synthesis methods.

3.2.3. Evolved star envelopes: contributions to the ISM. The matter lost from evolved stars becomes part of the ISM via planetary nebulae. It has usually been assumed that the molecular content of circumstellar shells is returned to the atomic state as the central star becomes a strong UV emitter, defining the planetary nebula stage. Yet, observations toward the Helix Nebula, which is 10000 years old, have demonstrated that $\mathrm{CO}$ is present in a large clumpy shell surrounding the central star and that $\mathrm{HCO}^{+}, \mathrm{HCN}, \mathrm{HNC}$ and $\mathrm{CN}$ exist as well (Bachiller et al 1997, Young et al 1999). Studies conducted recently by Tenenbaum et al (2009) have resulted in the detection of $\mathrm{CCH}, \mathrm{C}_{3} \mathrm{H}_{2}$ and $\mathrm{H}_{2} \mathrm{CO}$ in the Helix. Furthermore, mapping of $\mathrm{HCO}^{+}$and $\mathrm{H}_{2} \mathrm{CO}$ in the Helix suggests that these species are as widespread as CO (Zack et al 2012). All of these molecules have been studied via their $\mathrm{mm}$ rotational spectra. Higher energy transitions are likely to be found in these regions; recent laboratory spectroscopy (see, e.g., Lattanzi et al (2007)) will make such studies possible.

The discovery of complex molecules in old planetary nebulae is surprising; such species have been subjected to intense UV radiation for thousands of years. Theoretical calculations have shown that instabilities in the stellar wind 
can create finger-like clumps with densities as high as $10^{5} \mathrm{~cm}^{-3}$ (Redman et al 2003). Spitzer Space Telescope IR images show the presence of finger-like dust structures in the Helix (Hora et al 2006). Such clumps, composed of gas-phase molecules mixed with dust, become self-shielding. It could be that these clumps survive on sufficient timescales to bring molecular material to the diffuse ISM. Recent observations by Liszt and collaborators (see, e.g., Liszt et al (2006)) have demonstrated that polyatomic molecules such as $\mathrm{H}_{2} \mathrm{CO}, \mathrm{HCO}^{+}$and $\mathrm{C}_{3} \mathrm{H}_{2}$ are abundant in diffuse clouds.

The cycling of molecular material in the ISM has yet to be fully evaluated. Without knowledge of the gas-phase rotational spectra, our understanding of the molecular ISM would be negligible (Ziurys 2006). Furthermore, such spectra could not be interpreted without additional laboratory measurements or theoretical calculations. Knowledge of dipole moments, transition strengths, Einstein coefficients and energy levels are essential in the analysis of molecular data. Collisional crosssections, photodestruction rates and radiative absorption crosssections are also important. When combined, all these data enable detailed interpretation of gas-phase molecular spectra, producing a clearer picture of the nature of the dense ISM.

\subsubsection{Evolved stellar envelopes: fullerenes. Fullerene} molecules such as $\mathrm{C}_{60}$ and $\mathrm{C}_{70}$ have been prime observational targets ever since their discovery in laboratory experiments designed to simulate the chemistry of carbon star outflows (Kroto et al 1985). However it is only recently that observations with Spitzer have revealed for the first time the spectroscopic signatures of $\mathrm{C}_{60}$ and $\mathrm{C}_{70}$ in a variety of astronomical environments. The detections would not have been possible without spectroscopic data from laboratory measurements (Krätschmer et al 1990, Frum et al 1991, Martin et al 1993, Nemes et al 1994, Fabian 1996, Sogoshi et al 2000). The laboratory data provided the wavelengths and line strengths to confirm the astronomical detections.

$\mathrm{C}_{60}$ and $\mathrm{C}_{70}$ were first detected in a hydrogen-poor planetary nebula (Cami et al 2010). The hydrogen-poor conditions were thought to be necessary in light of laboratory measurements on fullerene production (De Vries et al 1993, Wang et al 1995). Other Spitzer observations reveal the presence of $\mathrm{C}_{60}$ in the reflection nebulae NGC 7023 and NGC 2023 (Sellgren et al 2010) and in planetary nebulae in our Galaxy and the Small Magellanic Cloud (SMC) (GarcíaHernández et al 2010). The latter work shows that the fullerenes are present in a variety of environments, including hydrogen-rich ones. García-Hernández et al (2010) suggest that the photochemistry of hydrogenated amorphous carbon plays a key role.

\subsection{Condensed matter physics}

3.3.1. Carbonaceous dust in outflows of late-type stars. Cosmic dust particles span a continuous size distribution from large molecules to $\mu \mathrm{m}$-sized particles and play an essential role in the evolution of the ISM (Tielens 2005). Carbonaceous dust particles are primarily formed in the outflow of carbon stars, through a combustion-like process where small carbon chains form PAHs that nucleate into larger-size PAHs and, ultimately, into nanoparticles (Henning and Salama 1998). According to this model, nucleation occurs for temperatures above $2000 \mathrm{~K}$, followed by the growth of amorphous carbon on the condensation nuclei in the $1500 \mathrm{~K}$ temperature range. As the temperature falls to around $1100 \mathrm{~K}$, aromatic molecules begin to form in the gas phase and condense onto the growing particles forming graphitic microstructures that will ultimately aggregate into larger structures such as seen in soot formation (Pascoli and Polleux 2000). Very little was known until recently about the formation of cosmic dust due to the difficulty of forming and isolating these large species and in tracking their evolutionary path under realistic astrophysical laboratory conditions. Much effort has been made in this direction leading to new laboratory tools and breakthrough results (Jager et al 2007, Ricketts et al 2011). Carbon pyrolysis and plasma-induced combustion experiments on mixtures of small hydrocarbons indicate that the product distribution is dominated by PAHs and partially hydrogenated PAHs. The condensates produced in the experiments consist of soot particles with graphene layers and PAHs. The formation process starts with small molecules recombining to form aromatic benzene rings, followed by the growth of larger PAHs through subsequent $\mathrm{C}_{2}$ addition to the aromatic rings and the final growth of grains by the condensation of large PAHs on the surfaces of the nuclei. These results demonstrate that lowtemperature condensation is a very likely formation process of soot and PAHs in AGB stars, confirming the model predictions.

\subsubsection{Silicates in envelopes of late-type stars. Silicates} are an important component of cosmic matter. Silicates form in the winds of AGB stars and are processed in the diffuse ISM. They are also an important component of dust in protoplanetary and debris disks where they help regulate thermal exchanges (Henning 2010). The detection at IR and millimeter wavelengths of silicate dust grains containing $\mathrm{O}$, $\mathrm{Si}, \mathrm{Fe}$ and $\mathrm{Mg}$, as well as some $\mathrm{Ca}$ and $\mathrm{Al}$, provides an important constraint on dust chemical composition and on grain size (Bouwman et al 2001, van Boekel et al 2005, Chiar and Tielens 2006, Sargent et al 2009, Juhasz et al 2009). Cosmic silicates are mostly found in the amorphous state, characterized by broad and structureless IR bands at 10 and $18 \mu \mathrm{m}$ that can be attributed to $\mathrm{Si}-\mathrm{O}$ stretching and $\mathrm{O}-\mathrm{Si}-\mathrm{O}$ bending modes, respectively (Draine 2003). In circumstellar environments, however, evidence for crystalline silicates is found both around (post-)AGB stars and in disks around Herbig Ae/Be stars, T Tauri stars and brown dwarfs (Henning 2010, Molster and Waters 2003). Silicates are also found in cometary environments (Crovisier et al 1997, Kelley and Wooden 2009, Hanner and Zolensky 2010), in spectra from asteroids (Emery et al 2006) and in interplanetary dust particles (Bradley 2010). These findings have only been possible thanks to vigorous laboratory programs that have helped characterize the basic properties of silicates that are needed to detect their signature in astronomical spectra. A vast amount of data resulting from laboratory studies dealing with both amorphous and crystalline silicates is now available in the literature, making it possible to derive information on topics as diverse as the evolution of 
cosmic dust, transport in protoplanetary and debris disks and redshifts in high- $z$ objects (for recent reviews see Henning (2010) and references therein).

\subsection{Plasma physics}

3.4.1. Ion heating in the solar corona and solar wind. UV spectroscopy of the solar corona has revealed that ion temperatures vary among species and that ion distribution functions are non-Maxwellian and anisotropic. These effects are most pronounced in certain minor ions and in particular increase with particle mass (Kohl et al 2006, Cranmer et al 2008, Landi and Cranmer 2009). These anisotropies may be a signature of heating by high-frequency turbulence, possibly driven by magnetic reconnection. Similar effects have been observed in laboratory plasmas. Brown et al (2002) reported an energetic ion population associated with 3D magnetic reconnection in the Swarthmore Spheromak Experiment device. Recently, ion heating associated with magnetic reconnection events in the Madison Symmetric Torus (MST) has been studied, revealing similar anisotropies and mass dependences (Tangri et al 2008, Fiksel et al 2009). The physical mechanism may be related to the reconnection driven turbulent cascade also recently studied on MST (Ren et al 2009). Thus, the experiments have shown that ions can be heated anisotropically, in a mass-dependent way, by MHD turbulence generated in reconnection events. This suggests that turbulent heating is responsible for the species-dependent temperature and anisotropy observed in the solar corona and that the turbulence could be generated by reconnection.

3.4.2. Reconnection in stars. Magnetic reconnection is a key process in stellar astrophysics. It is the leading candidate for the energy release mechanism in flares and may be an important mechanism for coronal heating. It must also occur in stellar interiors, as part of the magnetic dynamo. Laboratory experiments have made essential contributions to reconnection studies. Two recent review articles discuss these contributions in depth (Zweibel and Yamada 2009, Yamada et al 2010). Highlights include laboratory studies of flux rope dynamics, including reconnection in line tied plasmas and relaxation to a lower energy state (Bergerson et al 2006, Cothran et al 2009, Sun et al 2010), a criterion for the onset of fast collisionless reconnection mediated by the Hall effect (Yamada 2007) and studies of the electron diffusion layer, which clarify the mechanisms responsible for breaking the fieldlines and the apportionment of energy in the reconnection region (Ren et al 2008). These studies thus suggest a possible mechanism for triggering fast reconnection in solar flares and provide detailed information on how energy is apportioned among thermal and non-thermal electron and ion populations in solar reconnection.

\subsubsection{Stellar dynamos. Although magnetic cycles are} well established on the Sun and other stars, a theoretical explanation of stellar dynamos is still lacking and experimental confirmation is sparse. For many years, dynamo theory has been dominated by kinematic studies in which a mean field is built up from infinitesimal values by small-scale turbulence and large-scale shear. Recently, dynamo action has been reported in a number of liquid sodium experiments (Gailitus et al 2000, Monchaux et al 2007, Spence et al 2007). Liquid sodium, like stellar interior plasmas, is much more resistive than it is viscous. These experiments are being used to understand saturation mechanisms, the surprising role of turbulence in suppressing the growth of large-scale magnetic fields and the electromotive forces produced by large-scale and smallscale turbulent flows. These experiments are influencing the development of a new dynamo paradigm, in which dynamos are essentially non-linear and maintained by large-scale flows rather than small-scale turbulence.

3.4.4. Stellar opacities. Heating by fusion reactions deep within stellar cores produces thermal x-ray radiation and the outward transfer of this radiation is an essential element of stellar dynamics and evolution. The rate of attenuation of such radiation is the opacity. One can calculate opacities from fundamental atomic physics, but in even moderately complex elements such as iron this involves the interaction of many millions of transitions. Because of this complexity, calculations of opacity are uncertain and experimental measurements are essential to determine which calculations are correct. This has led to a quest to produce in the laboratory conditions present in stellar interiors so as to measure relevant opacities (Bailey et al 2009). During the 1990s, laboratory research and atomic theory resolved the issue of understanding pulsations in Cepheid variable stars (Rogers and Iglesias (1994), Springer et al (1997); see also the review by Remington et al (2006)). More recently, research has turned to the challenging issue of understanding solar structure. The Sun has an inner, radiative heat conduction zone that gives way to a convective zone nearer the surface. Solar models typically find a location of the boundary between these zones that differs from the measured one by more than 13 standard deviations (Basu and Antia 2008). One possible cause of this is knowledge of the energy-averaged opacity, which indeed must be accurate to $\sim 1 \%$ in order to fix the boundary to within the uncertainties of the observation (Bahcall et al 2004). By producing conditions of the stellar interior and measuring the detailed spectral structure of the opacity, researchers are now able to address this issue (Bailey et al 2007, 2009, Mancini et al 2009). These measurements showed that while very recent opacity models were nearly accurate enough under the conditions studied, previous opacity models were much less accurate. Challenges going forward are to produce accurate measurements in hotter, denser plasmas, in effect moving deeper into the Sun, while also addressing the other uncertainties discussed in section 3.1.2.

3.4.5. Photoionized gas. CVs are binary star systems composed of a white dwarf and (most often) a normal star. Mass from the normal star falls toward the white dwarf, producing a wide variety of phenomena. Recent laboratory work has focused on shock phenomena (Falize et al 2009a, 2009b, 2011) and on photoionization. CVs emit $\mathrm{x}$-ray radiation from the accreting matter. Such emission 
is also important in other accreting systems, for example, neutron stars, black holes and star-forming regions. The radiation photoionizes the nearby matter, producing plasma that is 'overionized' (ionized far beyond the level that would be produced by collisional ionization at the local electron temperature). One needs experiments to assess the accuracy of radiative rates across a wide range of transitions. An early effort in this direction (Foord et al 2004) used the radiation pulse produced by imploding a cylindrical array of metal wires to vaporize and then photoionize very thin foils of $\mathrm{Fe}$ and $\mathrm{NaF}$. They later compared the measured charge state distributions to those calculated by photoionization codes (Foord et al 2006), finding broad agreement but some differences. To obtain more uniform photoionized plasma, present-day experiments use radiation from an imploding wire array to create plasma in a gas cell (Bailey et al 2001, Mancini et al 2009). The radiation from a Z-pinch machine has also been used to photoionize a gas cell (Cohen et al 2003). In an alternative approach, a laser source is used to heat a gold cavity whose emission produces a moderately overionized plasma in a gas cell (Wang et al 2008). More recently, Fujioka et al (2009) used a laser-driven implosion to produce a $\sim 5 \mathrm{MK}$ blackbody radiation source, which in turn photoionized a laser-ablated, Si plasma. The photoionization experiments to date have shown that detailed comparisons of code results with laboratory data can improve our understanding of photoionized systems.

\subsubsection{Instabilities in type II supernovae. Core-collapse} $\mathrm{SNe}$ (ccSNe) involve much uncertain physics. Their complete physics and full range of dynamics are far beyond what can now be simulated. As a result, theories or simulations of these events must employ reduced physics, creating a need to test those simplified models. The potential for discovery is high, as unanticipated interactions of the physical processes may arise. Laboratory work relevant to ccSNe is currently limited to the 'late' phase of explosion, after the initial core collapse and after the shock wave forms that blows apart most of the star. It is now widely accepted that unstable mixing of stellar materials occurring during that phase is essential to explain observations of supernova SN 1987A (Arnett et al 1989, Chevalier 1992), but early simulations including these effects failed to do so (Muller et al 1991). This, combined with the observed asymmetry of SN ejecta, led to the hypothesis that such explosions were jet-driven (Wang et al 2001), although the mechanism that would cause this remains unidentified. Meanwhile, and quite unexpectedly, simulations employing improved traditional explosion models produced relevant levels of asymmetry (Kifonidis et al 2000, 2003, 2006, Guzman and Plewa 2009). This seems to be a nice story with an endpoint, yet all its elements remain uncertain without experimental evidence that other unanticipated coupling does not exist. Simulations cannot for example test the hypothesis that small-scale dynamics may feed back on the large-scale hydrodynamic evolution (Leith 1990).

Experiments have been developed to examine unstable hydrodynamics in a regime relevant to late-phase ccSNe dynamics. Work through 2005 is reviewed by Remington et al (2006). Such experiments can be well scaled in detail (Ryutov et al 1999) to local conditions in ccSNe. To date the large-scale behavior they have seen has been consistent with a variety of simulations (Kuranz et al 2009), showing that on a large scale our understanding of instabilities in the late phase of ccSNe is correct. However, to explain the observations it requires only that $\sim 1 \%$ of the inner material in the star finds a way to reach its outer layers with high velocity, and a number of small details have not been consistent between simulations and experiments (Calder et al 2002, Miles et al 2004, Kuranz et al 2010). Further work is seeking to understand the origin of the differences between observations and simulations, and to develop experiment designs relevant to the global dynamics of the explosion (Grosskopf et al 2009).

3.4.7. Radiative shocks in type II supernovae. During the explosion, the radiation pressure in the shocked matter produced by a type II SN exceeds the material pressure, but because the mean free path for thermal radiation is small compared with other scale lengths in the system, the shock wave behaves as a hydrodynamic shock with a polytropic index $\gamma=4 / 3$ (Ryutov et al 1999). This changes as the shock wave breaks out of the star and radiation can escape ahead of the shock. The shock enters a regime in which the thermal energy produced by the shock is mostly radiated away even though the layer behind the shock is many mean-free-path thick. A dense shell forms, which may be unstable (see sections 4.3.1 and 4.3.2). Current astrophysical instruments are beginning to observe such shock-breakout events (Calzavara and Matzner 2004, Chevalier and Fransson 2008, Soderberg et al 2008). Experiments have begun to produce and study shock waves in the same radiation-hydrodynamic regime as the shockbreakout events, with strong radiation emission, escape of the radiation ahead of the shock, and trapping of the radiation behind the shock (Bouquet et al 2004, Reighard et al 2006, Doss et al 2009, 2010). Such experiments are a subset of radiative-shock experiments more broadly (Bozier et al 1986, Grun et al 1991, Edwards et al 2001, Edens et al 2005, Hansen et al 2006, Koenig et al 2006, Busquet et al 2007). They typically involve producing a low atomic number $Z$ plasma 'piston' moving at $\gtrsim 100 \mathrm{~km} \mathrm{~s}^{-1}$ and using it to drive a shock wave in $\mathrm{Xe}$ or some other high- $Z$ gas. In the experiments the radiation transport is dominated by broadband thermal emission and absorption, while that in the star is more complex. Even so, the experiments are a vehicle to better understand the radiation-hydrodynamic behavior of this type of system and they have the potential to discover unanticipated behavior. To date, the experiments have shown that the Vishniac-like instability to which such dense shells are subjected is not so virulent as to greatly distort the shock. Ongoing work is developing scaling connections to $\mathrm{SNe}$ and SNRs (Doss 2011) and simulating the observed behavior (van der Holst et al 2011). These experiments constrain astrophysical simulation models, which cannot be expected to correctly model the SN if they cannot model these data.

3.4.8. Compact objects and gamma ray bursts: relativistic collisionless shocks. Most astrophysical shocks are collisionless shocks, in which electromagnetic turbulence 
randomizes the motion of the incoming particles, replacing the role of collisions in ordinary shocks. Astrophysical observations often imply that relativistic collisionless shocks must be present, as for example in gamma ray bursts (GRBs) (Piran 1999, Waxman 2006). Yet the observed emission from GRBs, attributed to synchrotron emission by electrons, required magnetic fields orders of magnitude larger than could be produced by mechanisms known to be present in the 1990s. The shocks involved are too complex to be fully described by a first-principles analytic or semi-analytic theory. The past decade has seen an explosion of work on such shocks (only some of which can be cited here), made possible primarily by the application of particle-in-cell (PIC) simulation methods on ever-larger computers. The first 3D PIC simulation of colliding electron-positron plasmas (Silva et al 2003), which are thought to occur in GRBs and elsewhere, found that the 3D Weibel instability produces both long-lived magnetic fields whose energy density is near that of the ions and non-thermal particle acceleration. This supported the theory (Medvedev and Loeb 1999) that the Weibel instability was the key process, which previously was only one of many theories. Further simulations studied initially unmagnetized (Spitkovsky 2008) and initially magnetized (Murphy et al 2010) electron-ion shocks, both also considered important in GRBs. In both cases one also sees the Weibel instability and the generation of strong magnetic fields, in addition to significant electron heating. The results of Murphy et al (2010) in combination with observations of polarization in emission from GRBs provide evidence for a significant primordial magnetic field in such events. Applying a similar model to relativistic electron-positron jets, Nishikawa et al (2009) found that the gamma-ray emission should come primarily from the shocked jet material rather than from the shocked ambient medium, confirming this interpretation of the observations of those objects. In this way large PIC simulations have become an important tool to advance understanding of relativistic astrophysical systems.

\subsection{Nuclear physics}

3.5.1. Nuclear synthesis via neutron capture. Elements beyond the iron peak are produced primarily by neutron (n) capture in the s- (slow) and r- (rapid) processes. The main s-process occurs in low-mass AGB stars while the weak s-process takes place in the He- and C-burning shells of massive stars. There is uncertainty about site or sites of the r-process, with Type II SNe (and their associated neutrino-driven winds) and neutron star mergers being leading candidates (Qian and Wasserburg 2007).

The fractional contributions of the weak and main sprocesses have been deduced from studies of solar system (including meteorite) abundances. The r-process must account for 'shielded' or other n-capture isotopes off the s-process path, and for other differences between observed abundances and those attributable to the s-process. Discussions can be found in Raiteri et al (1993), Arlandini et al (1999), The et al (2007) and Heil et al (2008). These studies attribute the light n-capture elements (e.g. Sr and $\mathrm{Zr}$ ) with high-mass stars and heavier s-process elements, such as Ba, with low-mass stars
(Busso et al 1999). Recent laboratory data for s-process crosssections are summarized by Käppeler et al (2011), updating Käppeler et al (1989).

Although the sites of the r-process are uncertain, data from metal-poor stars show that an r-process operated in the early galaxy at a frequency typical of ccSNe (Cowan and Thielemann 2004, Sneden et al 2008) (see also section 3.1.1). While properties of lighter r-process nuclei have been determined in the laboratory (Kratz et al 2000, Pfeiffer et al 2001, Möller et al 2003), much of the r-process path is through short-lived, very neutron-rich nuclei that are difficult to produce. Future facilities (e.g., the Facility for Rare Isotope Beams (FRIB)) will allow more extensive measurements of relevant masses and $\beta$-decay rates.

Interstellar abundances, however, do not appear to match solar system values. The abundances of $\mathrm{Ga}$ and $\mathrm{Ge}$ are $25 \%$ of the meteoritic value for low-density, warm gas, where depletion onto interstellar grains is expected to be minimal (Cartledge et al 2006, Ritchey et al 2011). The inferred Rb abundance is about $35 \%$ of the meteoritic value (Federman et al 2004, Walker et al 2009). The noble gas $\mathrm{Kr}$, which does not deplete onto grains, has an average abundance of $50 \%$ of the solar system value (Lodders 2003, Cartledge et al 2003). Ga, $\mathrm{Ge}, \mathrm{Kr}$ and $\mathrm{Rb}$ are predicted to form primarily in high-mass stars. In contrast, $\mathrm{Cd}$ and $\mathrm{Sn}$, which are mainly synthesized in low-mass stars, are not depleted for low density lines of sight (Sofia et al 1999), despite similarities between Ga, Ge, Rb, $\mathrm{Cd}$ and Sn condensation temperatures (Lodders 2003). The observed depletion patterns cannot be attributed to imprecise oscillator strengths, which are well known from laboratory and theoretical work (Morton 2000, 2003, Schectman et al 2000, Alonso-Medina et al 2005, Oliver and Hibbert 2010). Additional interstellar studies of other n-capture elements are needed.

3.5.2. Stellar nuclear fusion: pp chain. The proton-proton or pp chain is the principal mechanism by which low-mass hydrogen-burning stars like the Sun produce energy through $4 \mathrm{p} \rightarrow{ }^{4} \mathrm{He}+2 \mathrm{e}^{+}+2 v_{\mathrm{e}}$ where $\mathrm{e}^{+}$represents a positron and $v_{\mathrm{e}}$ an electron neutrino. The competition between the three cycles of the pp chain (ppI, ppII and ppIII) depends sensitively on the stellar core temperature, as the reactions require Coulomb barrier penetration, and on the specific rates of the reactions, which are conventionally given in terms of the astrophysical $S$-factor, from which the highly energydependent $S$-wave Coulomb behavior of the cross-section has been removed (Adelberger et al 2011). Laboratory measurements of $S$-factors are important to both solar neutrino physics and helioseismology. The uncertainties in laboratory $S$-factor measurements limit the precision of the standard solar model (SSM) neutrino flux and sound speed predictions (Bahcall et al 2005b). Associated astrophysics challenges include demonstrating through neutrino spectrum distortions that matter effects influence neutrino oscillations, detecting day-night effects and resolving discrepancies discussed in section 3.1.2 between the SSM and helioseismology measurements related to solar metallicity (Haxton and Serenelli 2008, Aharmin et al 2010, Abe et al 2011). 


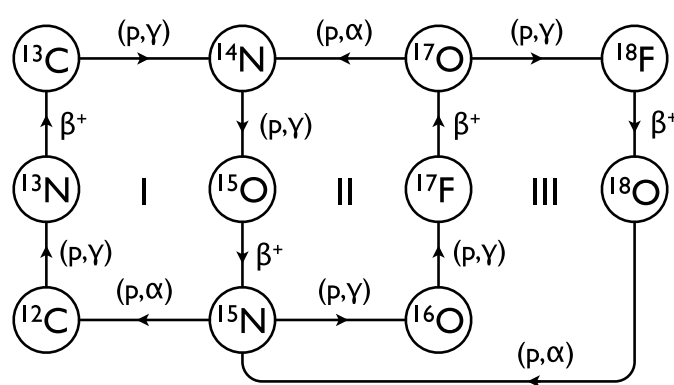

Figure 7. The three pathways that dominate the CNO cycles at lower temperatures. Recent experiments on the branch-point reactions involving ${ }^{15} \mathrm{~N}$ and ${ }^{17} \mathrm{O}$ are discussed in the text. Adapted from Wiescher et al (2010).

Recent key advances in laboratory astrophysics include a series of precise measurements of the reactions ${ }^{3} \mathrm{He}\left({ }^{3} \mathrm{He}\right.$, $2 \mathrm{p})^{4} \mathrm{He}$ (Bonetti et al 1999) and ${ }^{3} \mathrm{He}\left({ }^{4} \mathrm{He}, \gamma\right){ }^{7} \mathrm{Be}$ (Singh et al 2004, Bemmerer et al 2006a, Brown et al 2007, Confortola et al 2007, Gyurky et al 2007, di Leva et al 2009) which control the ratio of ppI solar neutrino flux to that of the ppII and ppIII. There have also been several new and precise measurements of ${ }^{7} \mathrm{Be}(\mathrm{p}, \gamma)^{8} \mathrm{~B}$ (Hammache et al 1998, 2001, Strieder et al 2001, Junghans et al 2002, 2003, 2010, Baby et al 2003a, 2003b), until recently the limiting nuclear physics uncertainty in predicting the flux of ppIII solar neutrinos. These measurements will have an important impact on the analysis of the currently running Borexino experiment (Arpsella et al 2008) which, in conjunction with the Sudbury Neutrino Observatory (SNO) (Aharmin et al 2010) and Super-Kamiokande (Abe et al 2011), will provide a direct test of matter effects on neutrino oscillations. They also impact the comparison between the total SSM ${ }^{8} \mathrm{~B}$ flux and that measured in SNO, which is sensitive to SSM parameters such as core metallicity. Recent progress in $S$-factor determinations came from technological advances like the Laboratory for Underground Nuclear Astrophysics (LUNA) (Costantini et al 2009, Broggini et al 2010), a specialized low-energy accelerator operating at great depth, allowing nearly background-free measurements of important cross-sections.

3.5.3. Stellar nuclear fusion: CNO cycle. Heavier hydrogen-burning stars produce their energy primarily through the carbon-nitrogen-oxygen (CNO) cycles, where nuclear reactions are characterized by higher Coulomb barriers. Hence energy production rises steeply with temperature, $\epsilon_{\mathrm{CNO}} \sim T^{18}$, compared with $\epsilon_{\mathrm{pp}} \sim T^{4}$ at solar temperatures. Unlike the pp chain, the CNO cycle requires pre-existing metals (in the astronomer's sense meaning all elements heavier than $\mathrm{He}$ ). These serve as catalysts for hydrogen burning, with the energy production at a fixed temperature proportional to metallicity. The CNO cycle is responsible for about $1 \%$ of solar energy generation, but dominates hydrogen burning in stars with central temperatures $\gtrsim 2 \times 10^{7} \mathrm{~K}$.

The rate-controlling step in the carbon-nitrogen $(\mathrm{CN})$ cycle, denoted by I in figure 7 , is ${ }^{14} \mathrm{~N}(\mathrm{p}, \gamma){ }^{15} \mathrm{O}$. The nuclear physics of this reaction is complex, with contributions from several ${ }^{15} \mathrm{O}$ resonances both above and below threshold. Work on this reaction over the past decade has been intense. New measurements have been made with both direct methods (Formicola et al 2004, Imbriani et al 2005, Runkle et al 2005, Bemmerer et al 2006b, Lemut et al 2006, Marta et al 2008) and indirect methods, covering the energy range between 70 and $480 \mathrm{keV}$ (see Wiescher et al (2010) and Adelberger et al (2011) for summaries). After summing all transitions $S_{114}^{\text {tot }}(0)=$ $1.66 \pm 0.12 \mathrm{keV}$ barn was obtained (Adelberger et al 2011), a value nearly a factor of two below previously recommended values. This has had significant consequences for astrophysics, such as increasing the age estimate for the oldest globular cluster stars by nearly a billion years (Runkle et al 2005). The increased precision of the $S$-factor will be critical to the analyses of data from the neutrino detector SNO+ (http://snoplus.phy.queensu.ca/Home.html) now under construction. By measuring the CNO neutrino flux, SNO+ may directly determine the carbon and nitrogen content of the solar core.

While ${ }^{14} \mathrm{~N}(\mathrm{p}, \gamma)$ controls the cycling rate of the $\mathrm{CN}$ cycle, other reactions determine the flow rate out of this cycle toward oxygen and heavier metals. The turn-on of these branches influences the opacity evolution and temperature profile of hydrogen-burning stars. Competition between ${ }^{15} \mathrm{~N}(\mathrm{p}, \alpha){ }^{12} \mathrm{C}$ and ${ }^{15} \mathrm{~N}(\mathrm{p}, \gamma){ }^{16} \mathrm{O}$ governs the division of the flow between the left two cycles illustrated in figure 7 . Here $\alpha$ represents an ${ }^{4} \mathrm{He}$ nucleus. Recent work on the second reaction (Bemmerer et al 2009) has led to corrections in earlier results (Adelberger et al 2011). The new measurements were done at novae energies and reduce the final nucleosynthesis yield of ${ }^{16} \mathrm{O}$ by up to $22 \%$, depending on the nova temperature (Bemmerer et al 2009). There is a similar competition between ${ }^{17} \mathrm{O}(\mathrm{p}, \alpha){ }^{14} \mathrm{~N}$, which closes CNO cycle II of figure 7 , and ${ }^{17} \mathrm{O}(\mathrm{p}, \gamma){ }^{18} \mathrm{~F}$, which leads either to the more complicated reaction network of the hot CNO cycles or to CNO I and II via ${ }^{18} \mathrm{~F}\left(\beta^{+} \nu\right){ }^{18} \mathrm{O}(\mathrm{p}, \alpha){ }^{15} \mathrm{~N}$. Recent work has led to significant cross-section changes affecting the flow toward the hot CNO cycle in novae (Fox et al 2005, Chafa et al 2007).

3.5.4. Stellar nuclear fusion: hot CNO burning. At temperatures greater than approximately a few times $10^{8} \mathrm{~K}$ a more complicated set of reactions allows mass flow to heavier nuclei (Wiescher et al 2010). In addition, the equilibrium abundances characterizing previously described cycles change: the rates of key radiative capture reactions increase to the point that they match or exceed those of the $\beta$ decays of ${ }^{13} \mathrm{~N}$ and ${ }^{15} \mathrm{O}$, for example, so that weak rates now govern the cycling time and rate of energy production, while rapid ( $\mathrm{p}, \gamma)$ reactions competing with $\beta$ decay open up new pathways. The hot $\mathrm{CNO}$ network involves reactions on unstable nuclei that require laboratory tools that only recently have become available, with the development of radioactive ion beam facilities. The resulting advances include the following.

- The cycle ${ }^{12} \mathrm{C}(\mathrm{p}, \gamma){ }^{13} \mathrm{~N}(\mathrm{p}, \gamma){ }^{14} \mathrm{O}\left(\beta^{+} v\right){ }^{14} \mathrm{~N}(\mathrm{p}, \gamma){ }^{15} \mathrm{O}$ $\left(\beta^{+} \nu\right)^{15} \mathrm{~N}(\mathrm{p}, \alpha)^{12} \mathrm{C}$ opens up when the radiative capture rate on ${ }^{13} \mathrm{~N}$ exceeds the $\beta$ decay rate. The key resonance governing the capture was measured in inverse kinematics, using an intense ${ }^{13} \mathrm{~N}$ radioactive beam of $3 \times 10^{8} \mathrm{~s}^{-1}$ (Decrock et al 1991, Delbar et al 1993). The direct capture 
contribution was recently determined from the asymptotic attenuation coefficient (Li et al 2006, Guo and Li 2007). These measurements together have led to a substantial increase in the recommended low-energy cross-section (Wiescher et al 2010), lowering the ignition temperature for the hot $\mathrm{CNO}$ cycle. This cross-section impacts models of novae, including the ${ }^{13} \mathrm{C} /{ }^{12} \mathrm{C}$ ratio in nova ejecta, as well as the predicted abundance of ${ }^{13} \mathrm{C}$, an important s-process neutron source (Arnould et al 1992).

- A critical branching in the hot $\mathrm{CNO}$ cycle depends on the competition between ${ }^{18} \mathrm{~F}(\mathrm{p}, \alpha){ }^{15} \mathrm{O}$ and ${ }^{18} \mathrm{~F}(\mathrm{p}, \gamma){ }^{19} \mathrm{Ne}$. These destruction channels for radioactive ${ }^{18} \mathrm{~F}$ are also the largest nuclear physics uncertainty affecting $\gamma$-ray emission from novae (Hernanz et al 1999). The development of high-intensity ${ }^{18} \mathrm{~F}$ beams of $\sim 10^{5}$ particles $\mathrm{s}^{-1}$ have allowed experimenters to determine the dominant ${ }^{18} \mathrm{~F}$ reaction rates for temperatures characterizing $\mathrm{ONeMg}$ novae (Bardayan et al 2000, 2002, Chipps et al 2009, Murphy et al 2009).

3.5.5. Core-collapse supernovae. Nuclear physics governs three important aspects of ccSNe, the core bounce (and ultimately the structure of the neutron star), energy transport and nucleosynthesis.

The core bounce depends on the nuclear EOS at densities that could range up to six times that of ordinary nuclear matter, at temperatures of tens of $\mathrm{MeV}$ and at extremes of isospin. The conditions at maximum compression are beyond the direct reach of experiment, but are constrained by astrophysical observations, including the stability of the $1.396 \mathrm{~ms}$ pulsar Terzan 5 (Hessels et al 2006) and the recent determination of a two-solar-mass neutron star measured by Shapiro delay (Demorest et al 2010) as well as by laboratory measurements of nuclear compressibilities. Laboratory measurements of giant monopole resonance energies in nuclei with and without neutron excesses constrain the compressibility for isospin symmetric matter and the symmetry energy $K_{\tau}$ critical to neutron-dominated matter (Piekarewicz 2010). New measurements, carried out in $\mathrm{Sn}$ isotopes, has led to $K_{\tau}=$ $-395 \pm 40 \mathrm{MeV}$ (Garg et al 2007), increasing the error bar on compressibility estimates (Piekarewicz 2010).

In a core-collapse supernova explosion the energy released through gravitational collapse must be preferentially transferred to the mantle of the star, to enable ejection. This is thought to be accomplished through the combined effects of the shock wave and neutrino heating. The neutrino heating and associated physics-neutrino opacity, neutrino cooling, $\beta$ decay rates important to lepton number emission and nucleosynthesis - are governed in part by nuclear GamowTeller and first-forbidden responses (Langanke and MartinezPinedo 2003). The Gamow-Teller responses have been mapped in the laboratory using forward-angle $(\mathrm{p}, \mathrm{n})$ and (n, p) scattering (Rapaport and Sugarbaker 1994) and then incorporated into nuclear models used in SN simulations. The resulting modern electron capture and $\beta$ decay rates have been found to increase the electron mass fraction $Y_{\mathrm{e}}$ throughout the iron core. As the size of the homologous core and thus the shock radius is proportional to $Y_{\mathrm{e}}^{2}$, this has significantly increased calculated shock wave strengths (Heger et al 2001, Bronson-Messer et al 2003). These improvements have also led to changes in neutrino $(v)$ process nucleosynthesis yields for key isotopes such as ${ }^{11} \mathrm{~B}$ and ${ }^{19} \mathrm{~F}$ (Heger et al 2005).

Recent studies of metal-poor halo stars (Cowan and Sneden 2006) have associated early Galaxy r-process events with ccSNe, which provide in their $v$-driven winds and mantles conditions under which an r-process might occur. The rate of nucleosynthesis is controlled by weak interactions, as new neutrons can be captured only after neutron holes are opened by $\beta$ decay. Thus the rate of $\beta$ decay is critical to determining which $\mathrm{SN}$ zones might be able to sustain the necessary nucleosynthetic conditions for the requisite time. Recent laboratory $\beta$ decay measurements for very-neutronrich isotopes near mass number $A=100$ have demonstrated that half-lives are a factor of two or more shorter than previously believed, which significantly relaxes constraints on the r-process time scale (Nishimura et al 2011).

\section{The galactic neighborhood}

The galactic neighborhood includes 'the structure and properties of the Milky Way and nearby galaxies and their stellar populations and evolution, as well as interstellar media and star clusters' (Blandford et al 2010a).

\subsection{Atomic physics}

4.1.1. Galactic chemical evolution. The early chemical evolution of the Galaxy can be studied from abundances of the iron-peak elements. These elements are synthesized in SN explosions and the stellar abundance trends with metallicities (i.e. $[\mathrm{Fe} / \mathrm{H}]$ ) provide important constraints on the explosion mechanisms of type II and Ia events. Early work by McWilliam (1997) demonstrated that as $[\mathrm{Fe} / \mathrm{H}]$ decreased below -2.4 , $\mathrm{Cr} / \mathrm{Fe}$ decreased while $\mathrm{Co} / \mathrm{Fe}$ increased, leading to a rising trend of $\mathrm{Cr} / \mathrm{Co}$ with decreasing $\mathrm{Fe} / \mathrm{H}$. This behavior provides clues to synthesis from $\mathrm{SNe}$ in the Galaxy as a function of metallicity. For example, models with $\alpha$-rich conditions tend to produce more elements heavier than $\mathrm{Fe}$, such as $\mathrm{Co}$, in contrast to lighter elements such as $\mathrm{Cr}$. It is also possible to reproduce these abundance trends by varying such effects as the explosion energies, neutron excess, mass cut position and progenitor masses in explosive SN nucleosynthesis. Additional studies have recently been completed, focusing on the iron-peak elements $\mathrm{Ti}, \mathrm{V}, \mathrm{Cr}, \mathrm{Mn}, \mathrm{Fe}, \mathrm{Co}$ and $\mathrm{Ni}$ as a function of $[\mathrm{Fe} / \mathrm{H}]$ (Henry et al 2010). The derived abundance trends have been based upon utilizing neutral (and less abundant) species for the Fe-peak element species and assuming LTE conditions.

Recent laboratory determinations of atomic data (e.g. oscillator strengths) have been obtained for $\mathrm{Cr}$ I (Sobeck et al 2007), CriI (Nilsson et al 2006), Mn I and II (den Hartog et al 2011), Co I (Nitz et al 1999) and Co II (Crespo LopezUrrutia et al 1994). These new experimental data have led to increasingly more accurate abundance values for the iron-peak elements in old halo stars. As a result of these new precise values, we are getting a clearer picture of the 
nature, and sources, of the earliest element formation in the Galaxy. In addition these new abundance values are providing increasingly stringent constraints on models (e.g. mass cut, energies, progenitor masses, elemental content of the ejecta, etc) of SN explosions and nucleosynthesis. Finally, an examination of the abundance trends of the iron-peak elements over different stellar metallicities is providing direct insight into the chemical evolution of the Galaxy.

\subsection{Molecular physics}

4.2.1. Interstellar medium chemical complexity. Recent developments in detector technology for ground-based measurements and the launch of the Herschel Space Observatory provide new opportunities to improve our understanding of interstellar chemistry. This has been particularly true for molecular ions and radicals which are important intermediate species in chemical networks describing the molecular evolution of interstellar clouds. Intermediates which have been detected spectroscopically include $\mathrm{SH}^{+}$(see section 5.2.1), $\mathrm{H}_{2} \mathrm{Cl}^{+}, \mathrm{OH}^{+}, \mathrm{H}_{2} \mathrm{O}^{+}$and $\mathrm{CH}^{+}$. Accurate transition frequencies are required for observational searches of these species, many of which have transitions at sub-millimeter and far-IR wavelengths. For $\mathrm{SH}^{+}$a combination of laser (Hovde and Saykally 1987), microwave (Savage et al 2004) and IR (Brown and Müller 2009) measurements provided the needed accuracy. The measurements in the THz range for $\mathrm{H}_{2} \mathrm{Cl}^{+}$by Araki et al (2001) yielded the required transition frequencies. The frequencies for $\mathrm{OH}^{+}$come from the study of Bekooy et al (1985). The $\mathrm{H}_{2} \mathrm{O}^{+}$frequencies are given by Mürtz et al (1998). $\mathrm{CH}^{+}$ data come from the spectroscopic work of Amano (2010). Lastly, there are numerous spectroscopic studies of $\mathrm{NH}$ and $\mathrm{NH}_{2}$, which have been compiled into the Cologne Database for Molecular Spectroscopy (Müller et al 2005).

Herschel has detected many of the above species. Lis et al (2010) discovered $\mathrm{H}_{2} \mathrm{Cl}^{+}$in absorption toward the star-forming region NGC 6334I in both ${ }^{37} \mathrm{Cl}$ and ${ }^{35} \mathrm{Cl}$ isotopologues. They found that the $\mathrm{HCl} / \mathrm{H}_{2} \mathrm{Cl}^{+}$ratios are consistent with chemical models, but the $\mathrm{H}_{2} \mathrm{Cl}^{+}$column densities greatly exceeded model predictions. The $\mathrm{OH}^{+}$and $\mathrm{H}_{2} \mathrm{O}^{+}$ions, which lead to $\mathrm{H}_{2} \mathrm{O}$ in ion-molecule chemical schemes, were seen in several star-forming clouds and the intervening diffuse clouds (see, e.g., Gupta et al (2010), Neufeld et al (2010) and Schilke et al (2010)). For example, Neufeld et al (2010) detected these ions in absorption toward the cloud W49N. The $\mathrm{OH}^{+} / \mathrm{H}_{2} \mathrm{O}^{+}$ abundance ratio indicated that the ions formed in clouds with small fractions of $\mathrm{H}_{2}$. Since these ions are produced by cosmic ray ionization of atomic and molecular hydrogen, an ionization rate could be inferred. The values are consistent with other recent determinations. Falgarone et al (2010) observed absorption from ${ }^{12} \mathrm{CH}^{+}$and ${ }^{13} \mathrm{CH}^{+}$. As the absorption from ${ }^{12} \mathrm{CH}^{+}$is optically thick, they were only able to set a lower limit of 35 on the isotope ratio. This value is consistent with other determinations of the ${ }^{12} \mathrm{C} /{ }^{13} \mathrm{C}$ ratio in ambient gas. Lastly, we note that Persson et al (2010) detected $\mathrm{NH}$ and $\mathrm{NH}_{2}$ in absorption in diffuse gas. Neither gas-phase nor grainsurface chemical models adequately explain the data; clearly further investigations into nitrogen chemistry are required.
4.2.2. Cosmic ray measurements. Energy input from Galactic cosmic rays, mainly relativistic protons and helium ions, drives important processes in the ISM. Ionization of $\mathrm{H}$ and $\mathrm{H}_{2}$ heats the gas and initiates chemical reactions. Cosmic rays interacting with the gas break apart ambient $\mathrm{C}, \mathrm{N}$ and $\mathrm{O}$ nuclei in a process called spallation, producing significant quantities of stable $\mathrm{Li}, \mathrm{Be}$ and $\mathrm{B}$ isotopes. The interactions with $\mathrm{H}$ and $\mathrm{H}_{2}$ also lead to $\gamma$-ray production through the decay of neutral pions. Many of these processes are dominated by low-energy cosmic rays (tens of $\mathrm{MeV}$ ), which are shielded from the Earth by the magnetic field of the Sun.

One way to obtain the cosmic ray ionization rate involves measurements of $\mathrm{H}_{3}^{+}$in diffuse molecular clouds (Snow and McCall 2006). The analysis is dependent on an accurate determination of the dissociative recombination rate coefficient, which until recently was poorly known. Measurements using storage rings (McCall et al 2003, Kreckel et al 2005, 2010, Tom et al 2009) and afterglows (Glosík et al 2008, 2009, Kotrík et al 2010), as well as theoretical calculations (Dos Santos et al 2007), are now converging on the most appropriate value for the rate coefficient. The cosmic ray ionization rate in diffuse molecular clouds inferred from $\mathrm{H}_{3}^{+}$observations is now more secure (e.g. Indriolo et al (2007)). One implication of this work is that the shape of the cosmic ray spectrum may differ from what has commonly been assumed (Indriolo et al 2009).

\subsection{Plasma physics}

4.3.1. Supernova remnants: radiative shock thermal instabilities. During the SN phase, a contact surface forms at the change in density gradient where the stellar envelope gives way to the stellar wind, between the driven forward shock and an eventual reverse shock. This contact surface is unstable and is subject to instabilities such as those discussed in sections 3.4.6 and 3.4.7. Other issues that arise in SNRs involve the predicted role of radiation. Here and in section 4.3.2 we discuss two of these issues.

As the shocks produced by $\mathrm{SNe}$ or other circumstances propagate across the ISM, the newly shocked material cools by the emission of radiation. The rate of cooling varies with temperature and there are regimes in which linear theory and simulations find that this produces an instability, causing oscillations in the shock velocity (Chevalier and Imamura 1982, Innes et al 1987, Kimoto and Chernoff 1997). Observational evidence of cooling that might be part of such an instability has been reported (Raymond et al 1991). The instability also would be expected to occur in accreting systems such as TW Hydrae and other T Tauri stars (Koldoba et al 2008), but recent observations find no evidence of it (Drake et al 2009, Gunther et al 2010). This creates a focused need for the observation of such instabilities in a laboratory environment, to show if they can in fact exist. This was accomplished (Hohenberger et al 2010) by the production of cylindrical shock waves by focusing a 1.4 ps laser pulse into a medium composed of Xe gas clusters (Moore et al 2008, Symes et al 2010). Measurements of the shock trajectory clearly showed velocity oscillations attributed to this 
instability. Future experiments can proceed toward systems that are more closely scaled to specific astrophysical cases.

\subsubsection{Supernova remnants: Vishniac instabilities. SNRs at} times produce very thin dense shells of material by radiative cooling, driven outward by the pressure within the SNR and decelerating as they accumulate more mass. Vishniac (1983) showed such shells to be unstable. Ryu and Vishniac (1991) showed that blast waves producing a density increase above about 10 to 1 are likewise unstable. This instability also may operate in other contexts where one finds a thin, dense shell, such as shocks emerging from $\mathrm{SNe}$ (see sections 3.4.6 and 3.4.7). Clumping in simulations of SNRs is often attributed to this process (van Veelen et al 2009). In observations, it is most often difficult to say whether the observed clumping is due to this instability as opposed to inhomogeneity in the medium being shocked (Grosdidier et al 1998) or to other instabilities such as Rayleigh-Taylor. However, the underlying theory is highly simplified, involving several assumptions including that the shell is infinitesimally thin and an unusual definition of the sound speed in the shell. This created the need for experimental tests. Experiments have produced the instability by driving a blast wave through $\mathrm{Xe}$ gas, generating the required large density increase by radiative cooling. Grun et al (1991) reported the first observation attributed to this process, but it was only recently that Edens et al (2005) reported a test of the predicted growth rate. Laming (2004) has discussed the common physics underlying these instabilities in astrophysical and laboratory systems and the connection of the Vishniac process with the thermal instability discussed above.

\subsubsection{Shock-clump interaction. High-resolution images} of astrophysical environments reveal that, in general, circumstellar and interstellar plasma distributions are essentially heterogeneous. Strong density perturbations over the ambient density, $\delta \rho / \rho_{\mathrm{amb}} \geqslant 1$, exist on a range of scales. The origin of such heterogeneity may lie in turbulent motions which exist in many astrophysical environments or through thermal or dynamical instabilities. Any supersonic flows through these environments will necessarily involve so-called shock-clump interactions. The importance of such clumpy flows cannot be understated as critical issues such as mixing, transport and global evolution will all differ in clumpy as opposed to smooth flows. The observational literature shows many clump studies addressing these issues in environments ranging from $\mathrm{SN}$ to active galactic nuclei (AGNs) (Smith and Morse 2004, Chugai and Chevalier 2006, Byun et al 2006, Westmoquette et al 2007, Fesen et al 2011).

Theoretical studies of shock-clump interactions have relied heavily on numerical simulations as the problem is essentially multi-dimensional and non-linear interactions dominate. Many studies of adiabatic shocks interacting with a single clump have been performed (see, e.g., Klein et al (1994)). Studies of magnetized and radiatively cooled single shocked clumps also exist but are fewer in number. Only a handful of multiple clump studies have been published (Fragile et al 2004, Yirak et al 2008). Because 3D simulation studies are often resolution limited (Yirak et al 2012) laboratory studies can offer relatively clean platforms for deeper exploration of shock-clump interactions. A robust literature reporting a host of shock-clump high energy density laboratory astrophysics (HEDLA) studies has emerged over the last decade.

The first HEDLA studies of shock-clump interactions focused on single clumps interacting with a passing shock (Kang et al 2000, Robey et al 2002, Klein et al 2003). These works, along with simulations and analytical work, were able to explore key features of shocked clump evolution including the breakup of downstream vortices by the Widnall instability. Characteristic density distributions of the clump as it is flattened by the passage of the shock along with break up of the vortex ring were well characterized in both experiments and simulations. The data shown in Klein et al (2003) were used to interpret the evolutionary stage of an observed structure in Puppis A by direct comparison with experimental data (Hwang et al (2005), see also figure 8). Recent studies have begun focusing on shock interactions with multiple clumps (Rosen et al 2009). Issues such as the interaction of bow shocks from nearby clumps as well as the effect of upstream clumps enhancing the breakup of downstream clumps in their dynamic shadow are currently being explored (Poludnenko et al 2004).

\section{Galaxies across time}

Galaxies across cosmic time covers 'the formation, evolution, and global properties of galaxies and galaxy clusters, as well as active galactic nuclei and [quasi-stellar objects (QSOs)], mergers, star formation rate, gas accretion, and supermassive black holes' (Blandford et al 2010a).

\subsection{Atomic physics}

5.1.1. Active galactic nuclei warm absorbers. Early Chandra and XMM-Newton observations of the AGN IRAS $13349+2438$ detected a new absorption feature in the 15-17 range (Sako et al 2003). This spectral feature is believed to originate in the warm absorber material surrounding the central supermassive black hole in AGNs and has since been observed in a number of other AGNs (see, e.g., Pounds et al (2001), Blustein et al (2002), Kaspi et al (2002, 2004), Behar et al (2003), Sako et al (2003), Steenbrugge et al (2003, 2005), Gallo et al (2004), Matsumoto et al (2004), Pounds et al (2004), Krongold et al (2005) and McKernan et al (2007)). These unresolved transition arrays (UTAs) were quickly identified as $2 \mathrm{p}-3 \mathrm{~d}$ innershell photoabsorption in iron M-shell ions (Sako et al 2003). New atomic calculations were soon carried out which demonstrated that the shapes, central wavelengths and equivalent widths of these features can be used to diagnose the properties of AGN warm absorbers such as wind and outflow velocities, ionization and elemental structure and mass loss rates and relative abundances (Behar et al 2001, Gu et al 2006). However, the ability to diagnose these properties was initially hindered by a lack of reliable ionization balance calculations, proper line identification and wavelengths and accurate absorption strengths.

Initial AGN models which matched absorption features from second- and third-row elements failed to correctly 


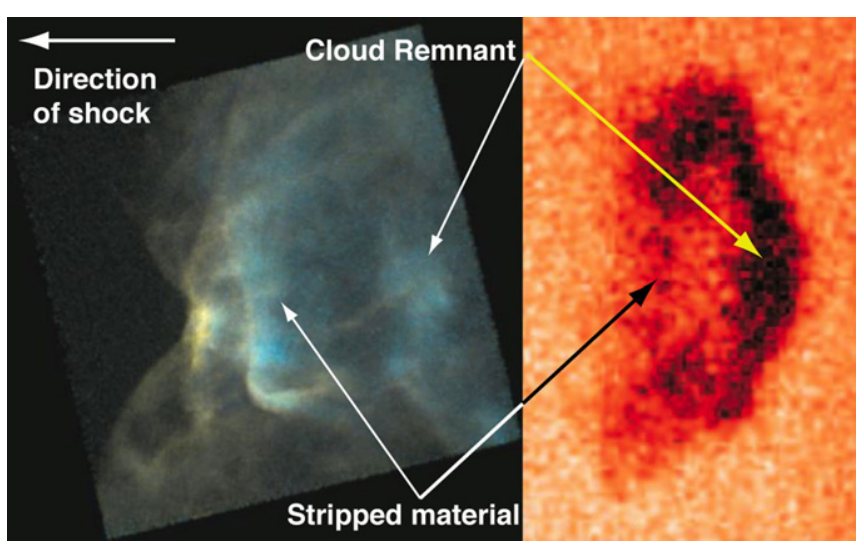

Figure 8. Astrophysical data from Chandra (left) and from the laboratory Nova laser (right) showing one phase of shock-clump interaction. Hwang et al (2005) used the laboratory data of Klein et al (2003) to interpret the astrophysical image. Adapted from Rosner and Hammer (2010)

reproduce the observed absorption from the fourth-row element iron (see, e.g., Netzer et al (2003)). The models predicted too high an iron ionization level. This was attributed to an underestimate in the models of the low temperature dielectronic recombination rate coefficients for the Fe M-shell ions (Netzer 2004, Kraemer et al 2004). This motivated a series of theoretical calculations ( $\mathrm{Gu}$ 2004, Badnell 2006a, 2006b, Altun et al 2006, 2007) and experimental studies (Schmidt et al 2006, 2008, Lukić et al 2007, Lestinsky et al 2009) which found dielectronic recombination rate coefficients up to orders of magnitude larger than the data previously available. These data improved agreement of the models with observations, though a number of issues still remain (Kallman 2010).

Comprehensive spectral models of the deep Chandra observation of the warm absorber in NGC 3783 suggested two ionization components in pressure equilibrium (Krongold et al 2003), with similar kinematic velocities. Netzer et al (2003) found three ionization components each with two sets of velocities and all three in pressure equilibrium. Subsequent theoretical calculations by $\mathrm{Gu}$ et al (2006) indicated only a single component in the wind, supporting the idea of pressure equilibrium (see section 5.1.2). Until recently, benchmark measurements capable of testing such boundbound photoabsorption calculations did not exist. This has now become possible with the use of a portable electron beam ion trap which can be coupled to third or fourth generation light sources (Epp et al 2007, Simon et al 2010). The results of Simon et al (2010) largely verified the calculation of $\mathrm{Gu}$ et al (2006) for Fexv. As a result of the photoabsorption work described here and the dielectronic recombination work mentioned above, more reliable models of AGN warm absorbers are now being developed. An example of this is discussed in section 5.1.2.

\subsubsection{Thermal stability of active galactic nuclei emission line} regions. Many models of the origin of the emission lines of AGNs have been proposed (see chapter 14 of Osterbrock and Ferland (2006), hereafter AGN3). Possibilities include winds from stars or the accretion disk, an ionized layer above the surface of the disk, or distinct clouds confined by a surrounding

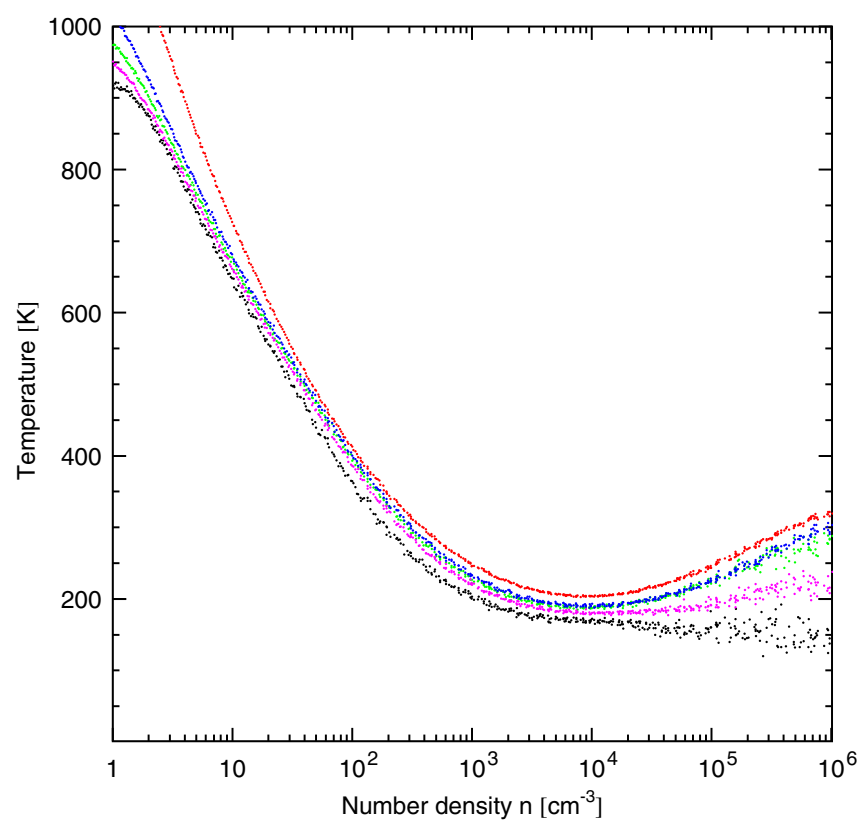

Figure 9. Evolution of a primordial cloud evolving in an initially ionized protogalactic halo using various associative detachment rate coefficients (Kreckel et al 2010). Each point corresponds to a spherical shell of material surrounding the center of the cloud. The black and red data use the previous upper and lower limits for the associative detachment reaction as discussed in Glover et al (2006). The green data use the experimentally benchmarked theoretical results of Kreckel et al (2010), while the magenta and blue data use a rate coefficient, respectively, $25 \%$ larger and smaller than this. During this epoch the Jeans mass is set by density at the minimum temperature reached, leading to a factor of 20 uncertainty with the old data and a factor of only 2 with the new.

hot medium. If the latter is the case, then the gas phases where clouds can exist are determined by the thermal cooling curve. This is the relationship between the gas temperature and the cooling rate (AGN3, chapter 3 ). If gas pressure equilibrium applies, then regions with very different kinetic temperatures and hydrogen densities can exist at the same gas pressure. This scenario dates back to early work done on the ISM (Field et al 1969) and was revived by Krolik et al (1981) for AGNs.

The form of the cooling curve results from massive amounts of atomic data. Collisional excitation and radiative decay rates are needed for thousands of lines while collisional and photoionization rates, together with radiative, dielectronic and charge transfer recombination rate coefficients, are needed for hundreds of ions. Dielectronic recombination is the most uncertain of these rates. Improvements in the dielectronic recombination data, mainly from storage ring measurements and expanded theory, have significantly affected our understanding of the stable phases (see the reviews of Schippers (2009) and Schippers et al (2010)). Atomic theory and experiment are now in far better agreement for the dielectronic recombination data with significantly larger low temperature rate coefficients than those of the previous generation.

Chakravorty et al $(2008,2009)$ revisited the thermal stability of AGNs using an updated version of the spectral simulation code Cloudy (Ferland et al 1998). This code uses, among many data sources, the compilation of recombination 
rates from Badnell et al (2003) and Badnell (2006c). Chakravorty et al (2008, 2009) found that the updated dielectronic recombination rates produced significant changes in the predicted distribution of ions. The shape of the stability curve changed significantly as a result. These changes were large enough that the existence of certain gas phases were affected with implications for the final spectrum. However, the modern dielectronic recombination data do not extend to the low-charge, multi-electron systems that are needed to fully understand AGN clouds. This remains an outstanding need.

\subsection{Molecular physics}

5.2.1. Chemistry surrounding active galactic nuclei. Chemical models of $\mathrm{x}$-ray dominated regions (XDRs) surrounding AGNs and YSOs (Maloney et al 1996) reveal significant abundances of doubly charged ions to be cospatial with $\mathrm{H}_{2}$. The role of doubly charged ions as a diagnostic has been actively pursued since then. Dalgarno (1976) pointed out the potential importance of reactions involving these ions. Recently, Abel et al (2008) considered the effects on AGNs. Laboratory studies show that some $\mathrm{X}^{2+}+\mathrm{H}_{2}$ reactions occur rapidly at elevated temperatures. Chen et al (2003) measured a total rate coefficient for the reaction $\mathrm{S}^{2+}+\mathrm{H}_{2}$, while Gao and Kwong (2003) studied the reaction $\mathrm{C}^{2+}+\mathrm{H}_{2}$. Neither study, however, determined branching fractions among the various final chemical channels. Abel et al (2008) estimated what branching fractions would yield an observable effect on the $\mathrm{SH}^{+}$chemistry. They found that as long as the branch to $\mathrm{SH}^{+}+$ $\mathrm{H}$ was a few percent, doubly ionized chemistry would be the dominant pathway for $\mathrm{SH}^{+}$production. They also showed that $\mathrm{S}^{2+}$ was effectively destroyed once $\mathrm{H}_{2}$ forms and that the $\mathrm{S}^{2+}$ abundance remains high in gas dominated by atomic hydrogen and not only in ionized gas as was previously thought. A key consequence of their calculations is that much of the mid-IR emission from [S III] at 18.7 and $33.5 \mu \mathrm{m}$ may come from the XDR and not the ionized gas associated with an AGN. Recent detections of $\mathrm{SH}^{+}$in our Galaxy (Menten et al 2011) suggest the possibility for observing this molecular ion elsewhere and using the proposed diagnostics of Abel et al (2008).

\section{Cosmology and fundamental physics}

Cosmology and fundamental physics includes 'the early universe, the microwave background, the reionization and galaxy formation up to virialization of protogalaxies, largescale structure, the intergalactic medium, the determination of cosmological parameters, dark matter, dark energy, tests of gravity, astronomically determined physical constants, and high energy physics using astronomical messengers' (Blandford et al 2010a).

\subsection{Atomic physics}

6.1.1. Primordial abundances. The abundances of the primordial elements $\mathrm{H}, \mathrm{D},{ }^{3} \mathrm{He},{ }^{4} \mathrm{He}$ and ${ }^{7} \mathrm{Li}$ provide a key test of Big Bang cosmology. The data are taken from neutral gas in the Lyman-alpha forest for $\mathrm{D}, \mathrm{H}$ II regions both within the Galaxy $\left({ }^{3} \mathrm{He}\right)$ and outside $\left({ }^{4} \mathrm{He}\right)$, and observations of metal-poor stars for ${ }^{7} \mathrm{Li}$ (Steigman 2011). Corrections are made for the effects of post Big Bang nucleosynthesis (BBN) processing. For example, D and ${ }^{7} \mathrm{Li}$ are burned in stellar environments, and ${ }^{7} \mathrm{Li}$ is synthesized in cosmic ray interactions with nuclei in the ISM. For a recent discussion, see Charbonnel et al (2010).

The primordial ${ }^{4} \mathrm{He}$ abundance is usually measured in giant $\mathrm{H}_{\text {II }}$ regions or dwarf irregular galaxies. In these extragalactic emission nebulae, $\mathrm{H}$ and $\mathrm{He}$ are photoionized. Corrections for stellar production of ${ }^{4} \mathrm{He}$ are determined from correlations with metallicity. A recent examination of 93 spectra for 86 low-metallicity extragalactic $\mathrm{H}$ II regions showed a linear dependence of ${ }^{4} \mathrm{He}$ on $\mathrm{O} / \mathrm{H}$, and yielded an extrapolated zero-metallicity ${ }^{4} \mathrm{He}$ mass fraction of $0.2565 \pm 0.0010$ (stat) \pm 0.0050 (syst) (Izotov and Thuan 2010). Others have advocated more conservative errors (Aver et al 2010).

Accurate ${ }^{4} \mathrm{He} / \mathrm{H}$ determinations from ratios of optical recombination lines require precise photo-production rates for electron recombination with $\mathrm{H}^{+}$(Storey and Hummer 1995) and ${ }^{4} \mathrm{He}^{+}$. Recent atomic calculations for the two-electron system ${ }^{4} \mathrm{He}$ I (Benjamin et al 1999, Bauman et al 2005, Porter et al 2007) are in good agreement. Remaining issues include collisional processes involving the ground or metastable levels, photoionization cross-sections for non-hydrogenic moderate$n$, small- $l$ levels and transition probabilities for these levels (Porter et al 2009).

The abundance of $\mathrm{D}$ is important because of its sensitivity to the baryon-to-photon ratio $\eta_{\mathrm{B}}$, varying as $\eta_{\mathrm{B}}^{-1.6}$. From a limited set of high-red shift, low-metallicity QSO absorption line systems, $\log (\mathrm{D} / \mathrm{H})=-4.55 \pm 0.04$ was found (Pettini et al 2008), in good agreement with the Wilkinson Microwave Anisotropy Probe (WMAP) determinations of $\eta_{\mathrm{B}}$.

The observations of ${ }^{7} \mathrm{Li}$ in the atmospheres of old halo stars is constant to within measurement errors of $5 \%$ over a variety of masses and metallicities. While lithium is fragile in stellar environments, a well-formed 'plateau' is found at low metallicity, yielding an abundance ${ }^{7} \mathrm{Li} / \mathrm{H}=\left(1.23_{-0.16}^{+0.34}\right) \times$ $10^{-10}$ (Ryan et al 2000) that is about a factor of four below BBN predictions based on the WMAP $\eta_{\mathrm{B}}$. Any astrophysical explanation of this anomaly would have to account for the stability of the plateau.

6.1.2. Protogalaxy and first star formation. In the early universe during the formation of protogalaxies and the first stars, commonly called Population III stars, $\mathrm{H}^{-}$ plays an important role in the formation of $\mathrm{H}_{2}$, as is described in section 6.2.1. $\mathrm{H}_{2}$ is an important coolant leading to the formation of structure during this epoch and reliable predictions of the $\mathrm{H}^{-}$abundance are critical for reliable cosmological models. $\mathrm{H}^{-}$can be destroyed by photodetachment

$$
\mathrm{H}^{-}+\gamma \rightarrow \mathrm{H}+\mathrm{e}^{-}
$$

and by mutual neutralization

$$
\mathrm{H}^{+}+\mathrm{H}^{-} \rightarrow \mathrm{H}+\mathrm{H} \text {. }
$$

These processes decrease the $\mathrm{H}^{-}$abundance, thereby limiting the amount of $\mathrm{H}_{2}$ which forms and correspondingly reducing the cooling of the primordial gas. 
Recent theoretical work has been carried out for each of these reactions. Miyake et al (2010) have calculated new photodetachment rates taking into account both the $\mathrm{H}^{-}$resonance states lying near $11 \mathrm{eV}$ and radiation fields characteristic of Population III stars, blackbody sources, power-law spectra and the hydrogen Lyman modulated sawtooth spectra of the high-redshift intergalactic medium. Stenrup et al (2009) have recently calculated new mutual neutralization data valid for temperatures relevant during protogalaxy and first star formation. Their results agree with previous theoretical calculations to within $30-40 \%$ (Bates and Lewis 1955, Fussen and Kubach 1986), but are about a factor of 2-3 smaller than the experimental results of Moseley et al (1970), suggesting the need for further experimental work.

\subsection{Molecular physics}

6.2.1. Protogalaxy and first star formation. Ro-vibrational collisional excitation of $\mathrm{H}_{2}$ followed by radiative relaxation is an important cooling mechanism in the early universe. $\mathrm{H}_{2}$ is formed during this epoch by the associative detachment reaction

$$
\mathrm{H}+\mathrm{H}^{-} \rightarrow \mathrm{H}_{2}^{-} \rightarrow \mathrm{H}_{2}+\mathrm{e}^{-} .
$$

$\mathrm{H}_{2}$ formation, in turn, can be limited by reactions (1) and (2), both of which compete with reaction (3) for $\mathrm{H}^{-}$anions.

Until recently, there was nearly an order-of-magnitude uncertainty in the rate coefficient for reaction (3) (Glover et al 2006). This uncertainty severely limited our ability to model protogalaxies and metal-free stars forming from initially ionized gas, such as in ionized regions (i.e., $\mathrm{H}$ II regions) created by earlier Population III stars (Glover et al 2006, Glover and Abel 2008, Kreckel et al 2010). Recently, measurements for this reaction have been carried out using a mergedbeam apparatus leading to an experimentally benchmarked theoretical rate coefficient with an uncertainty of $\pm 24 \%$ (Bruhns et al 2010a, 2010b, Kreckel et al 2010). As a result, for example, the uncertainty in the model-predicted Population III Jeans mass due to errors in the atomic data has decreased from a factor of 20 to 2 (Kreckel et al 2010) (see also figure 9). As a result of all the experimental and theoretical work described here and in section 6.1.2, we are significantly closer to the point where remaining uncertainties in models for protogalaxy and first star formation tell us something about cosmology and not about the underlying chemistry.

\subsection{Nuclear physics}

6.3.1. Big Bang nucleosynthesis. The comparison between $\mathrm{BBN}$ calculations and primordial abundances is a cornerstone of modern cosmology, determining $\eta_{B}$ (now confirmed by $W M A P$ ), and limiting the baryonic matter contribution to the universe to about $4 \%$ of the closure density (Olive 1999). Thus most of the dark matter is non-baryonic. BBN in combination with inventories of the matter in stars, inter-cluster diffuse gas, and the intergalactic medium indicate that a significant fraction $(\gtrsim 25 \%)$ of the baryonic matter is non-luminous (Silk 1999, Bregman 2007).

$\mathrm{BBN}$ calculations depend on the Maxwellian-averaged nuclear cross-sections for the various reactions of figure 10 .

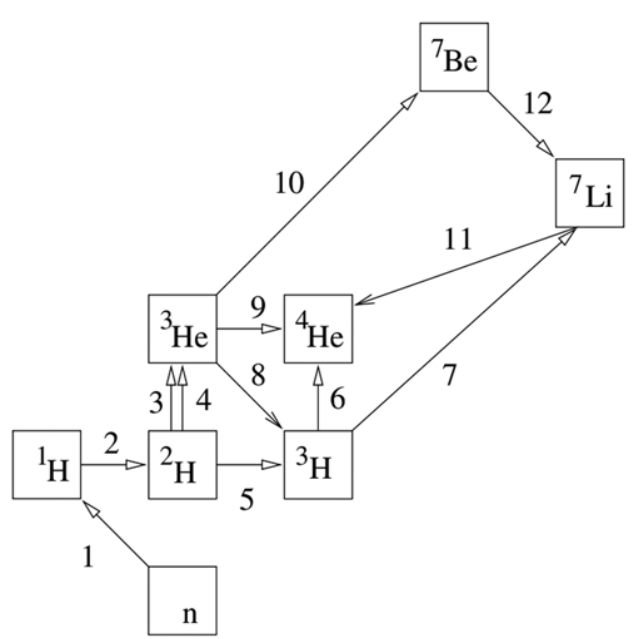

1. $p \longleftrightarrow n$

2. $p(n, \gamma) d$

3. $d(p, \gamma)^{3} \mathrm{He}$

4. $d(d, n)^{3} \mathrm{He}$

5. $d(d, p) t$

6. $t(d, n)^{4} \mathrm{He}$

7. $t(\alpha, \gamma)^{7} \mathrm{Li}$

8. ${ }^{3} \mathrm{He}(n, p) t$

9. ${ }^{3} \mathrm{He}(d, p){ }^{4} \mathrm{He}$

10. ${ }^{3} \mathrm{He}(\alpha, \gamma){ }^{7} \mathrm{Be}$

11. ${ }^{7} \mathrm{Li}(p, \alpha){ }^{4} \mathrm{He}$

12. ${ }^{7} \mathrm{Be}(n, p){ }^{7} \mathrm{Li}$

Figure 10. The reaction network for BBN, from Nollett and Burles (2000).

Comprehensive efforts have been made to assess the effects of cross-section uncertainties on BBN predictions (see, e.g., Nollett and Burles (2000) and Coc and Vangioni (2010)). Table 1 of Coc and Vangioni (2010) describes the impact of key nuclear physics uncertainties on the abundances of ${ }^{4} \mathrm{He}, \mathrm{D},{ }^{3} \mathrm{He}$ and ${ }^{7} \mathrm{Li}$, given the WMAP value of $\eta_{\mathrm{B}}$. The ${ }^{4} \mathrm{He}$ yield is sensitive to weak rates now well constrained by neutron $\beta$ decay (Lopez and Turner 1999). The reaction $\mathrm{n}+\mathrm{p} \rightarrow \mathrm{D}+\gamma$ has a large impact on ${ }^{7} \mathrm{Li}$ by competing with ${ }^{7} \mathrm{Be}(\mathrm{n}, \mathrm{p}){ }^{7} \mathrm{Li}(\mathrm{p}, \alpha){ }^{4} \mathrm{He}$ for neutrons: ${ }^{7} \mathrm{Li}$ is synthesized as ${ }^{7} \mathrm{Be}$ at the WMAP value for $\eta_{B}$. While there are meager lowenergy data on this reaction, calculations based on effective field theory (EFT) treatments are believed to be accurate to $1 \%$ (Chen and Savage 1999, Ando et al 2006). New measurements (Tornow et al 2003) of the inverse reaction, made at energies of 2.39-4.05 MeV, are in excellent agreement with EFT predictions. ${ }^{7} \mathrm{Li}$ is also sensitive to the production channel rate for ${ }^{3} \mathrm{He}(\alpha, \gamma){ }^{7} \mathrm{Be}$. Four new data sets, summarized in Adelberger et al (2011), have now determined this crosssection to $\pm 5.2 \%$. Recent measurements (Leonard et al 2006) of a third reaction important to ${ }^{7} \mathrm{Li},{ }^{2} \mathrm{H}(\mathrm{d}, \mathrm{p}){ }^{3} \mathrm{H}$, confirm earlier parameterizations of this cross-section.

Recent work has not uncovered a nuclear physics explanation for the discrepancy between BBN predictions and the ${ }^{7} \mathrm{Li}$ abundance determined from metal-poor stars. For a discussion, see Chakraborty et al (2010) and references therein.

6.3.2. Ultra-high-energy cosmic rays and neutrinos. Recent instrumentation advances in high-energy astrophysics include the Pierre Auger Observatory (Pierre Auger Collaboration 2010), for the study of ultra-high-energy (UHE) cosmic rays, the IceCube Observatory, a South Pole high-energy neutrino detector scheduled for completion in 2011 (Abbasi et al 2010), and prototype UHE neutrino detectors, such as the Antarctic Impulsive Transient Antenna (ANITA) experiment (Barwick et al 2006) and the Radio Ice Cherenkov Experiment (RICE) (Kravchencko et al 2006).

The Pierre Auger program includes measurements of the spectrum, anisotropies and composition of UHE cosmic rays, including at the GZK cutoff (Greisen 1966, Zatsepin 
and Kuz'min 1966) of $\sim 5 \times 10^{19} \mathrm{eV}$. Interactions with the cosmic microwave background (CMB) limit the distances UHE protons/nuclei can travel. Interactions with the CMB and with IR, optical and UV background photons are well constrained by a large database of laboratory nuclear physics. The energy-loss mechanisms include single and multiple pion production off the proton, nuclear reactions such as photodisintegration, photo-pair processes and photoabsorption followed by re-emission. References to propagation models based on this input physics can be found in Kotera and Olinto (2011).

A key objective of the Pierre Auger science program, determining the primary energy and mass of UHE cosmic rays, requires a detailed model of the interactions of cosmic ray protons and nuclei with nuclei in the upper atmosphere. Cosmic rays above $10^{14} \mathrm{eV}$ are measured indirectly, through the cascades of secondary particles that result from their atmospheric collisions. The energy and composition of the incident UHE cosmic ray are determined by comparing the observed extensive air showers with those predicted by models. Center-of-mass energies near the GZK cutoff are two orders of magnitude beyond the limits of our highest energy machines, the Large Hadron Collidor (LHC) and the Relativistic Heavy Ion Collidor (RHIC). Thus significant extrapolations are required. For a discussion of the uncertainties, see Alessandro et al (2011). Recent tests of existing codes against first LHC data are described in d'Enterria et al (2011).

Cosmic ray neutrinos are a tool for probing the universe at asymptotic energies and distances and for identifying point sources, as neutrinos are not deflected by magnetic fields. IceCube was designed to detect neutrinos with energies between $10^{10}$ and $10^{17} \mathrm{eV}$, through the Cerenkov light emitted by charged particles they produce. The extension to higher energies, required to detect the neutrinos from the nuclear reactions responsible for the GZK cutoff, requires ice volumes a factor $\sim 100$ beyond IceCube's $\mathrm{km}^{3}$, as well as new detection techniques. Methods under development are based on coherent radio emission, the Askaryan effect (Askaryan 1962, Askaryan et al 1979). Recent laboratory tests of the Askaryan effect using targets of silica and rock salt confirmed that radio emission provides a means of detecting UHE neutrinos (Saltzberg et al 2001, Gorham et al 2005).

\subsection{Particle physics}

6.4.1. Baryon number asymmetry: experiment. The explanation for the excess of baryons over antibaryons in the early universe, and thus a non-zero $\eta_{\mathrm{B}}$, is a key puzzle in cosmology. Baryogenesis requires charge-parity (CP) violation and baryon number violation. $\mathrm{CP}$ violation arises in the standard model through the Cabibbo-Kobayashi-Maskawa (CKM) phase and through the quantum chromodynamics (QCD) $\bar{\theta}$ parameter, and has been observed in the laboratory in kaon decays and at the $\mathrm{B}$ factories. However, the known $\mathrm{CP}$ violation is not sufficient to account for the baryon number asymmetry. Baryon number violation has not been seen in the laboratory, despite intense effort.

Static electric dipole moments (EDMs) require CP violation. As there is a significant gap between current experimental bounds on EDMs and standardmodel predictions based on the CKM phase, detection of an EDM might indicate a new source of $\mathrm{CP}$ violation relevant to baryogenesis. Current limits come from atomic beam experiments on the electron EDM, $\left|d_{\mathrm{e}}\right|<$ $1.6 \times 10^{-27} \mathrm{ecm}$ (Commins et al 1994), and from trap experiments with ultracold neutrons, $\left|d_{\mathrm{n}}\right|<2.9 \times 10^{-26} \mathrm{e} \mathrm{cm}$ (Baker et al 2006). Alternatively, neutron and proton EDMs as well as $\mathrm{CP}$-violating nucleon-nucleon (NN) interactions can be probed in neutral atoms. The ${ }^{199} \mathrm{Hg}$ vapor-cell experiment, $\left|d\left({ }^{199} \mathrm{Hg}\right)\right|<3.1 \times 10^{-29} \mathrm{e} \mathrm{cm}$, provides the most stringent limits on the proton and quark chromo EDMs, and on the strength of scalar, pseudoscalar and tensor CP-violating semileptonic interactions (Griffith et al 2009).

Baryogenesis could have arisen from the decays of heavy right-handed neutrinos, with the symmetry violation communicated to the baryons through mechanisms within the standard model (so-called 'sphalerons'; Fukugita and Yanagida (1986)). Recent laboratory discoveries-non-zero neutrino masses and two large mixing angles-have made this scenario quite plausible. The CP-violating observable is proportional to a product that involves the three mixing angles and the Dirac CP phase. A great deal of laboratory effort is now focused on both short- and long-baseline neutrino oscillation experiments to measure the third mixing angle and to detect leptonic $\mathrm{CP}$ violation at low energies by comparing neutrino oscillation channels, e.g. $v_{\mu} \rightarrow v_{\mathrm{e}}$ and $\bar{v}_{\mu} \rightarrow \bar{v}_{\mathrm{e}}$. Experiments in progress include the Daya Bay (Lin 2011) and Double Chooz (Palomares 2009) reactor experiments, and the Tokai-to-Kamioka (T2K) long-baseline neutrino oscillation experiment (Rubbia 2011). FermiLab 'intensity frontier' plans include a search for neutrino CP violation (see http://www.fnal.gov/pub/science/experiments/intensity/).

Laboratory limits on baryon number violation come from proton decay searches. The Super-Kamiokande Collaboration (Nishino et al 2009) has placed limits on the partial lifetimes for modes favored by minimal SU(5) Grand Unified Theories (GUTs), $\mathrm{p} \rightarrow \mathrm{e}^{+} \pi^{0}$ and $\mathrm{p} \rightarrow \mu^{+} \pi^{0}$, of $8.2 \times 10^{33} \mathrm{yr}$ and $6.6 \times 10^{33} \mathrm{yr}$, respectively, at a $90 \%$ confidence level. The collaboration has also established (Kobayashi et al 2005) stringent limits on modes favored by supersymmetric GUTs, $\mathrm{p} \rightarrow \mu^{+} K^{0}, \mathrm{n} \rightarrow \bar{v} K^{0}, \mathrm{p} \rightarrow \mu^{+} K^{0}$, and $\mathrm{p} \rightarrow \mathrm{e}^{+} K^{0}$ of $2.23 \times 10^{34} \mathrm{yr}, 1.3 \times 10^{32} \mathrm{yr}, 1.3 \times 10^{33} \mathrm{yr}$ and $1.0 \times 10^{33} \mathrm{yr}$, respectively.

6.4.2. Baryon number asymmetry: theory. In theory, no major paradigm shift has occurred in the last ten years. (For a review of baryogenesis models, see Dine and Kusenko (2003).) However, considerable progress has been made in refining the predictions of various scenarios and new possibilities have been proposed. In one class of models, the baryon asymmetry is produced at the electro-weak phase transition, as a result of new physics at the electro-weak scale, such as supersymmetry. While the basic scenario for electro-weak baryogenesis (EWB) was described long ago (Kuzmin et al 1985), recent developments include a re-evaluation (Lee et al 2005) of the relevant source terms which bias the production of a net baryon number via sphaleron transitions (Huet and 
Nelson 1996) and of the associated resonant relaxation effects (Lee et al 2005). Also, it was realized that the supersymmetric parameter which is space compatible with the production of enough baryon asymmetry possesses a two-resonances structure (Cirigliano et al 2006, 2010). One of the two resonances corresponds to the scenario of 'bino-driven' EWB, where the origin of dark matter is deeply connected with that of the baryon asymmetry (Li et al 2009).

As possible experimental EWB tests, it was recently pointed out that the EDM size for the electron and for the neutron is bounded from below in EWB ( $\mathrm{Li}$ et al 2010), as a result of unavoidable electro-weak two-loop contributions (Li et al 2008). The issue of producing a strong enough firstorder phase transition in supersymmetry (Carena et al 2009) has also been investigated, together with the possibility of enhancing the first-order character altering the Higgs sector, for instance adding a singlet scalar field (Pietroni 1993, Apreda et al 2002, Profumo et al 2007). Questions related to the gauge dependence of criteria identifying strong enough first-order EW phase transitions have also been recently studied (Patel and Ramsey-Musolf 2011).

Numerous recent efforts targeted the "coincidence problem' given by the ratio of the baryonic density $\Omega_{\mathrm{b}}$ to non-baryonic dark matter density $\Omega_{\mathrm{DM}}$ being of order unity $\left(\Omega_{\mathrm{DM}} / \Omega_{\mathrm{b}} \sim 5\right)$. A variety of proposals have been recently put forward, including darkogenesis (Shelton and Zurek 2010), xogenesis (Buckley and Randall 2010) and hylogenesis (Davoudias et al 2010) that for reasons of space we cannot review here.

Remarkable progress has also been made on the front of leptogenesis models (for a comprehensive review see Giudice et al (2004)). Recent developments include the flavordynamics of leptogenesis (Pilaftsis 2005) and resonant leptogenesis near the electroweak phase transition (Pilaftsis and Underwood 2005). Some of these models might be testable with the LHC and with experiments sensitive to lepton-number and/or leptonflavor violation (Pilaftsis 2009).

\subsubsection{Direct dark matter detection. A wide spread} experimental campaign is afoot to search for signatures of Galactic dark matter scattering off ordinary matter nucleons. These efforts are theoretically motivated by various compelling considerations (Goodman and Witten 1985) and typically target weakly interacting massive particles (WIMPs), although axion searches have also been very active in the last decade (Duffy and van Bibber 2009).

WIMPs can undergo elastic or inelastic scattering processes with nucleons (in the latter case exciting or ionizing the target atom, or producing the nuclear emission of a photon). The possibility of WIMPs transitioning themselves to an excited state has also been envisioned (Tucker-Smith and Weiner 2001). We briefly review here elastic dark matter scattering only, a process that can occur via spin-dependent or spin-independent interactions. The nuclear recoil induced by WIMP scattering can produce light (scintillation), charge (ionization) and/or phonon (heat) signals. In practice, current generation direct detection experiments are typically sensitive to two or more of these signals, with the aim of achieving the best possible background rejection. Experiments that make use of scintillation and ionization include for instance XENON (Aprile et al 2010) and ZEPLIN (ZonEd Proportional scintillation in LIquid Noble gases; Akimov et al (2010)); among those that use scintillation and heat is CRESST (Cryogenic Rare Event Search with Superconducting Thermometers) (Angloher et al 2008), while CDMS (Cryogenic Dark Matter Search) (Akerib et al 2006) and EDELWEISS (Expérience pour DEtecter Les Wimps En Site Souterrain) (Gerbier 2010) make use of both ionization and heat. Other experimental setups that make use of one channel only include the scintillation experiment DAMA/LIBRA (DArk MAtter/Large sodium Iodide Bulk for RAre processes) (Bernabei et al 2010) or the ionization experiment CoGeNT (Contact Germanium Neutrino Telescope) (Aalseth et al 2008). Interestingly, the latter two experiments recently reported controversial signals that have been attributed to Galactic dark matter (Fitzpatrick et al 2010).

The first positive direct detection signal has been reported by the DAMA collaboration, with a rather impressive total exposure of 1.17 ton-yr (combining DAMA/NaI (DArk MAtter/Sodium-Iodine Target) and DAMA/LIBRA), which quotes an annual modulation in the recoil energy range of $2-6 \mathrm{keV}$ electron equivalent at the $8.9 \sigma$ confidence level (Bernabei et al 2010). The WIMP elastic-scattering interpretation of this signal is largely inconsistent with limits reported by XENON (Angle et al 2008) and CDMS (CDMS II Collaboration 2010). The CoGeNT experiment reported an exponential-like excess of events in the few keV energy range, compatible with a light-mass WIMP (Aalseth et al 2011). Anomalous events have also been reported by CRESST and CDMS, although with relatively low statistical significance. Figure 11 presents a sample of recent experimental and theoretical results on direct dark matter detection on the plane defined by the WIMP mass and the spin-independent WIMP-proton scattering cross-section. The regions shaded in light red are compatible with the DAMA/LIBRA modulation signal (Bernabei et al 2010), while the theoretical expectation for the scattering cross-section in the constrained minimal supersymmetric Standard Model (CMSSM) is shown with the blue and green shaded areas (see Trotta et al (2008) for more details). The figure also shows selected experimental limits, that rule out the corresponding upper-right corners of the plot. The limits shown are from the CDMS (dark blue), ZEPLIN (light blue) and CoGeNT (red dotted) experiments. We also indicate the projected reach of ton-size class experiments with a black dotted line. In summary, in the last ten years the field of direct dark matter searches reached a stage of full maturity. It is exploring interesting regions of theoretically favored parameter space and tantalizing signals are emerging from more than one experiment.

6.4.4. Indirect dark matter detection. Many theoretically motivated models for dark matter, including weakly interacting massive particles WIMPs, predict that dark matter pairannihilates into ordinary Standard Model particles. Searches for the annihilation debris of dark matter are generically dubbed 'indirect dark matter detection'. In the last decade, 


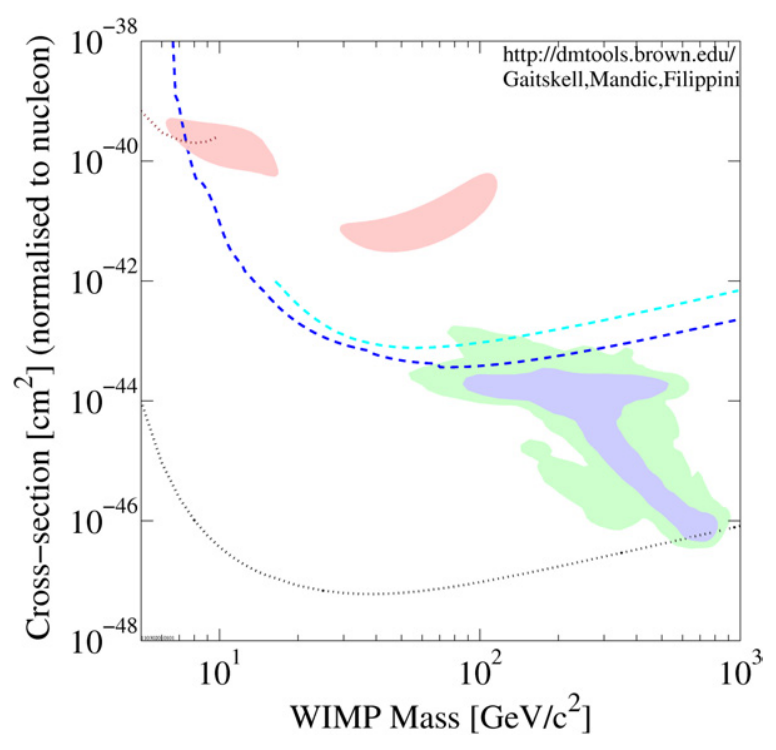

Figure 11. The plane of dark matter-proton spin-independent cross-section versus mass. The dark and light blue lines indicate constraints from CDMS and ZEPLIN, respectively, while the red dotted line is from CoGeNT: parameter space points above the lines are experimentally excluded. The light red areas represent regions compatible with the positive annual modulation signal from DAMA/LIBRA (Bernabei et al 2010). The light green and blue regions indicate theoretical predictions for the $95 \%$ and $68 \%$ confidence level regions of CMSSM parameter space as determined in Trotta et al (2008). Plot obtained through http://dmtools.brown.edu.

indirect detection has been one of the primary science goals of several new experiments and telescopes looking for highenergy gamma rays, neutrinos and antimatter.

Most notably, the Fermi gamma-ray space telescope (Atwood et al 2009) sets significant limits on the pairannihilation rate of dark matter from the non-observation, in gamma rays, of nearby dwarf spheroidal galaxies (Abdo et al 2010a), of clusters of galaxies (Ackermann et al 2010a) and of monochromatic gamma-ray lines (Abdo et al 2010b). Atmospheric Cherenkov telescopes, such as the Very Energetic Radiation Imaging Telescope Array System (VERITAS), the High Energy Stereoscopic System (HESS) and the Major Atmospheric Imaging Cherenkov (MAGIC) telescope, have also produced interesting limits, for higher mass dark matter candidates (Aharonian et al 2006). Construction of the IceCube neutrino telescope at the South Pole was recently completed and the IceCube collaboration has delivered the first limits on dark matter annihilation in the Galactic Center (Abbasi et al 2010, 2011) and from particles captured from the center of the Sun (Heros 2010).

Anomalies in the high-energy flux of cosmic ray positrons, including the rising positron fraction measured by the Payload for Antimatter Matter Exploration and Light-nuclei Astrophysics (PAMELA) satellite (Adriani et al 2009) in the $10-100 \mathrm{GeV}$ range and the hard positron-plus-electron flux reported by Fermi (Ackermann et al 2010b), triggered a great deal of excitement as possible signatures of dark matter annihilation (Arkani-Hamed et al 2009) or decay (Arvanitaki et al 2009). Astrophysical explanations, including nearby mature pulsars (Profumo 2008) as well as in situ secondary particle acceleration (Blasi 2009), have also been put forward as plausible counterparts to the cosmic-ray electron-positron anomalies.

Other signatures that have been associated with Galactic dark matter annihilation include the WMAP haze (Hooper et al 2007), a diffuse radio emission that could be related to electrons and positrons produced by dark matter and possibly a gammaray haze (Dobler et al 2010). The evidence for the latter has been questioned (Linden and Profumo 2010). Recent reanalyses point toward two giant gamma-ray 'bubbles' whose morphology appears incompatible with a dark matter origin (Su et al 2010).

6.4.5. Dark matter theory. The last decade has seen giant leaps in theoretical studies concerning dark matter. On the one hand, simulation of structure formation in collisionless cold dark matter cosmologies has achieved unprecedented resolution and level of detail; on the other hand, model building inspired by possible experimental signals or by pure theoretical arguments has triggered interesting new particle physics scenarios.

In the field of $N$-body simulations, which only include gravitationally interacting dark matter, three milestones, among several other exciting simulations, have been the Millennium (Springel et al 2005), Via Lactea (Diemand et al 2007) and Bolshoi (Klypin et al 2011) simulations. While Millennium, in 2005, provided the basis for hundreds of studies on statistical properties of dark matter halos and on models for galaxy formation in a cosmological setting (including mock catalogues and merger trees), Bolshoi (completed in 2010) uses an updated set of cosmological parameters and will play a similar role in the immediate future. The Via Lactea suite of simulations specialized on Milky-Way-size dark matter structure, with important implications for indirect (Diemand et al 2007) and direct (Kuhlen et al 2010) dark matter searches. An important issue that will dominate future studies of the dark matter distribution is the effect of baryons on the dark matter density profiles (Duffy et al 2010).

On the model-building frontier, numerous studies explored in detail the phenomenology of supersymmetric models in collider, direct and indirect detection (Baer et al 2005). Several groups focused on statistical analyses of the supersymmetric (SUSY) parameter space, based upon, e.g., a Bayesian approach (Trotta et al 2008). Numerous theoretical model-building efforts concentrated on explaining observed anomalies in dark matter search experiments. These include leptophilic models (Fox and Poppitz 2009), models with a Sommerfeld enhancement at low dark matter relative velocities (Pospelov et al 2008), discussed to account for claimed indirect detection signals, and inelastic (Tucker-Smith and Weiner 2001) dark matter models, proposed to interpret direct dark matter signals.

\section{Discussion and outlook for the future}

Our astrophysical understanding of the cosmos continues to be propelled forward by advances in laboratory astrophysics. This review has touched on many, but far from all, of the 
achievements of the past decade. The coming decade promises to be equally, if not more, fruitful. The Astro 2010 Survey Report and Panel Reports (Blandford et al 2010a, 2010b) have laid out a series of exciting scientific objectives, the achievement of which they point out is going to require numerous advances in laboratory astrophysics. We direct the reader to those reports for a detailed discussion.

Additional in depth discussions about the laboratory astrophysics needs and opportunities for the coming decade can be found in a number of white papers written over the past few years. These include those submitted by the Working Group on Laboratory Astrophysics (WGLA) to the Astro 2010 Survey (Brickhouse et al 2009a, 2009b, 2009c, 2009d, 2009e) as well as community input to Astro 2010 through the Science White Papers (http://sites.nationalacademies.org/BPA/BPA_050603) and the Laboratory Astrophysics White Papers (http://sites.nationalacademies.org/BPA/BPA_051118). Another white paper is that submitted by the WGLA to the US National Research Council Planetary Science Decadal Survey: 2013-2022 (Gudipati et al 2009). In plasma laboratory astrophysics, there have been a couple of reports recently released by the community (Prager et al 2010, Rosner and Hammer 2010). And most recently there is the white paper from the 2010 Laboratory Astrophysics Workshop sponsored by the Astrophysics Division of the Science Mission Directorate which covered atomic, molecular, condensed matter and plasma laboratory astrophysics (Savin et al 2011). These all point the way to the future and the richness of discovery which we can only just begin to guess.

\section{Acknowledgments}

The authors thank their many colleagues including J E Bailey, P Beiersdorfer, G V Brown, J R Crespo López-Urrutia, H Ji, H Kreckel, J E Lawler, M Medvedev, T Plewa, D Sasselov, R K Smith, C Sneden, B J Wargelin and S Widicus Weaver for stimulating conversations. NSB was supported in part by the NASA contract NAS8-03060 to the Smithsonian Astrophysical Observatory for the Chandra X-ray Center. JJC is supported in part by the National Science Foundation through grant AST 0707447. RPD acknowledges support from DOE/NNSA Defense Sciences and Advanced Scientific Computing, from DOE/Science Office of Fusion Energy Sciences and from the Defense Threat Reduction Agency. GJF acknowledges support by NSF (0908877), NASA (07-ATFP07-0124, 10ATP10-0053 and 10-ADAP10-0073) and STScI (HST-AR12125.01 and HST-GO-12309). MSG acknowledges funding from NASA Astrobiology Institute 'Icy Worlds' and support from the Jet Propulsion Laboratory, California Institute of Technology, under a contract with the National Aeronautics and Space Administration. WCH was supported in part by the US Department of Energy under grant DE-SC00046548 to the University of California at Berkeley. EH acknowledges the support of NASA through its program in laboratory astrophysics and through the Herschel program. SP is partly supported by the US Department of Energy with an Outstanding Junior Investigator Award and by Contract DEFG02-04ER41268 and by NSF Grant PHY-0757911. FS acknowledges the support of the Astrophysics Research and Analysis Program of NASA Science Mission Directorate. DWS is supported in part by the NASA Astronomy and Physics Research and Analysis program, the NASA Solar Heliospheric Physics program and the NSF Division of Astronomical Sciences Astronomy and Astrophysics Grants program. EGZ was supported in part by the NSF grant PHY-0821899 to the University of Wisconsin.

\section{Appendix. Acronyms}

A complete list of the acronyms used throughout the text is given in table $\mathrm{A} 1$.

Table A1. List of acronyms used in the text.

\begin{tabular}{|c|c|}
\hline Acronym & Phrase \\
\hline AGB & asymptotic giant branch \\
\hline $\mathrm{AGN}$ & active galactic nucleus \\
\hline AGNs & active galactic nuclei \\
\hline ANITA & ANtarctic Impulsive Transient Antenna \\
\hline $\mathrm{BBN}$ & Big Bang nucleosynthesis \\
\hline ccSNe & core collapse supernovae \\
\hline CDMS & Cryogenic Dark Matter Search \\
\hline CKM & Cabibbo-Kobayashi-Maskawa \\
\hline $\mathrm{CMB}$ & cosmic microwave background \\
\hline $\mathrm{CN}$ & carbon-nitrogen \\
\hline $\mathrm{CNO}$ & carbon-nitrogen-oxygen \\
\hline CoGeNT & Contact Germanium Neutrino Telescope \\
\hline $\mathrm{CP}$ & charge-parity \\
\hline CMSSM & $\begin{array}{l}\text { constrained minimal supersymmetric } \\
\text { Standard Model }\end{array}$ \\
\hline CRESST & $\begin{array}{l}\text { Cryogenic Rare Event Search with } \\
\text { Superconducting Thermometers }\end{array}$ \\
\hline $\mathrm{CVs}$ & cataclysmic variables \\
\hline DAMA/LIBRA & $\begin{array}{l}\text { DArk MAtter/Large sodium Iodide Bulk } \\
\text { for RAre processes }\end{array}$ \\
\hline DAMA/NaI & DArk MAtter/sodium-iodine target \\
\hline DIB & diffuse interstallar absorption band \\
\hline EDELWEISS & $\begin{array}{l}\text { Expérience pour DEtecter Les Wimps } \\
\text { En Site Souterrain }\end{array}$ \\
\hline EDM & electric dipole moment \\
\hline EOS & equations of state \\
\hline EWB & electro-weak baryogenesis \\
\hline FRIB & Facility for Rare Isotope Beams \\
\hline FTIR & Fourier transform infrared \\
\hline GRB & gamma ray burst \\
\hline GUT & Grand Unified Theory \\
\hline GZK & Greisen-Zatsepin-Kuz'min \\
\hline HEDLA & High Energy Density Laboratory Astrophysics \\
\hline HESS & High Energy Stereoscopic System \\
\hline IAU & International Astronomical Union \\
\hline IR & infrared \\
\hline ISM & interstellar medium \\
\hline $\mathrm{KBO}$ & Kuiper belt object \\
\hline $\mathrm{LHC}$ & Large Hadron Collidor \\
\hline LMC & Large Magellanic Cloud \\
\hline LTE & local thermodynamic equilibrium \\
\hline LUNA & $\begin{array}{l}\text { Laboratory for Underground } \\
\text { Nuclear Astrophysics }\end{array}$ \\
\hline MAGIC & Major Atmospheric Imaging Cherenkov \\
\hline MHD & magnetohydrodynamic \\
\hline MRI & magnetorotational instability \\
\hline
\end{tabular}


Table A1. (Continued.)

\begin{tabular}{ll} 
MST & Madison Symmetric Torus \\
NN & nucleon-nucleon \\
PAH & polycyclic aromatic hydrocarbon \\
PAMELA & Payload for Antimatter \\
& Matter Exploration and Light-nuclei \\
& Astrophysics \\
PIC & particle-in-cell \\
QCD & quantum chromodynamics \\
QSO & quasi-stellar object \\
RHIC & Relativistic Heavy Ion Collidor \\
RICE & Radio Ice Cherenkov Experiment \\
SEM-EDX & scanning electron microscopy using \\
& energy-dispersive x-ray \\
SMC & Small Magellanic Cloud \\
SN & supernova \\
SNe & supernovae \\
SNO & Sudbury Neutrino Observatory \\
SNR & supernova remnant \\
SSM & standard solar model \\
SUSY & supersymmetric \\
TEM & transmission electron microscopy \\
TMC & Taurus molecular cloud \\
TNO & trans Neptunian object \\
TOF-SIMS & time of flight secondary ion \\
& mass spectrometry \\
TRACE & Transition Region And Coronal Explorer \\
T2K & Tokai-to-Kamioka \\
UHE & ultra-high-energy \\
UIR & unidentified infrared \\
UTA & unresolved transition array \\
UV & ultraviolet \\
VERITAS & Very Energetic Radiation Imaging \\
& Telescope Array System \\
YSO & young stellar object \\
WGLA & Working Group on Laboratory \\
& Astrophysics \\
WIMP & weakly interacting massive particles \\
WMAP & Wilkinson Microwave \\
& Anisotropy Probe \\
XDR & x-ray dominated region \\
XMM & X-ray Multi-Mirror mission \\
ZEPLIN & ZonEd Proportional scintillation \\
& in LIquid Noble gases \\
3D & three-dimensional \\
\hline &
\end{tabular}

\section{References}

Aalseth C E et al 2008 Phys. Rev. Lett. 101251301

Aalseth C E et al 2011 Phys. Rev. Lett. 106131301

Abbasi R et al 2010 Astrophys. J. 73218

Abbasi R et al 2011 Phys. Rev. D 84022004

Abe K et al 2011 Phys. Rev. D 83052010

Abel N P, Federman S R and Stancil P C 2008 Astrophys. J. 675 L81-L84

Abdo A A et al 2010a Astrophys. J. 712 147-58

Abdo A A et al 2010b Phys. Rev. Lett. 104091302

Ackermann M et al 2010a J. Cosmol. Astropart. Phys. 1005025

Ackermann M et al 2010b Phys. Rev. D 82092004

Adelberger E G et al 2011 Rev. Mod. Phys. 83 195-245

Adriani O et al 2009 Nature 458 607-9

Agúndez M, Cernicharo J and Gulin M 2007 Astrophys. J. 662 L91-L94

Agúndez M, Cernicharo J and Goicoechea J R 2008a Astron. Astrophys. 483 831-7

Agúndez M, Cernicharo J, Pardo J, Fonfría Expósito J P, Guélin M, Tenenbaum E D, Ziurys L M and Apponi A J 2008b Astrophys. Space Sci. 313 229-33
Agúndez M et al 2010 Astron. Astrophys. 517 L2

Aharmim B et al 2010 Phys. Rev. C 81055504

Aharonian F et al 2006 Phys. Rev. Lett. 97221102

A'Hearn M F 2008 Space Sci. Rev. 138 237-46

Akimov D Y et al 2010 Phys. Lett. B 692 180-3

Akerib D S et al 2006 Phys. Rev. Lett. 96011302

Alessandro B et al 2011 arXiv:1101.1852

Allamandola L J, Tielens A G G M and Barker J R 1989 Astrophys. J. Suppl. Ser. 71 733-75

Alonso-Medina A, Colón C and Rivero C 2005 Phys. Scr. 71 154-8

Altun Z, Yumak A, Badnell N R, Loch S D and Pindzola M S 2006 Astron. Astrophys. 447 1165-74

Altun Z, Yumak A, Yavuz I, Badnell N R, Loch S D and Pindzola M S 2007 Astron. Astrophys. 474 1051-9

Amano T 2010 Astrophys. J. 716 L1-L3

Anderson H M, den Hartog E A and Lawler J E 1996 J. Opt. Soc. Am. B 13 2382-91

Ando S, Cyburt R H, Hong S W and Hyun C H 2006 Phys. Rev. C 74025809

Angloher $\mathrm{G}$ et al 2008 The CRESST dark matter search IDM2008: Identification of Dark Matter (Stockholm, Sweden, 18-22 Aug. 2008) 014

Angle J et al 2008 Phys. Rev. Lett. 100021303

Apponi A J, Barclay W L Jr and Ziurys L M 1993 Astrophys. J. 414 L129-L132

Apreda R, Maggiore M, Nicolis A and Riotto A 2002 Nucl. Phys. B $631342-68$

Aprile E and Profumo S 2009 New J. Phys. 11105002

Aprile E et al 2010 Phys. Rev. A 105131302

Araki M, Furuya T and Saito S 2001 J. Mol. Spectrosc. 210 132-6

Arkani-Hamed N, Finkbeiner D P, Slatyer T R and Weiner N 2009 Phys. Rev. D 79015014

Arlandini C, Käppeler F, Wisshak K, Gallino R, Lugaro M, Busso M and Straniero O 1999 Astrophys. J. 525 886-900

Arnett W D, Bahcall J N, Kirschner R P and Woolsey S E 1989 Annu. Rev. Astron. Astrophys. 27 629-700

Arnould M, Paulus G and Jorissen A 1992 Astron. Astrophys. 254 L9-L12

Arpesella C et al 2008 Phys. Rev. Lett. 101091302

Arvanitaki A, Dimopoulos S, Dubovsky S, Graham P W, Harnik R and Rajendran S 2009 Phys. Rev. D 80055011

Askaryan G A 1962 Sov. Phys._JETP 14 441-3

Askaryan G A, Dolgoshein B A, Kalinovsky A N and Mokhov N V 1979 Nucl. Instrum. Methods 164 267-78

Asplund M, Grevesse N, Sauval A J, Allende Prieto C and Kiselman D 2004 Astron. Astrophys. 417 751-68

Atwood W B et al 2009 Astrophys. J. 697 1071-102

Aver E, Olive K A and Skillman E D $2010 \mathrm{~J}$. Cosmol. Astropart. Phys. JCAP5(2010)003

Baby L T et al 2003a Phys. Rev. Lett. 90022501

Baby L T et al 2003b Phys. Rev. C 67065805

Bachiller R, Forveille T, Huggins P J and Cox P 1997 Astron. Astrophys. 324 1123-34

Bacmann A, Lefloch B, Ceccarelli C, Castets A, Steinarker J and Loinard L 2002 Astron. Astrophys. 389 L6-L10

Bacmann A, Lefloch B, Ceccarelli C, Steinacker J, Castets A and Loinard L 2003 Astrophys. J. 585 L55-L58

Badenes C, Borkowski K, Hughes J P, Hwang U and Bravo E 2006 Astrophys. J. 645 1373-91

Badenes C, Hughes J P, Cassam-Chenaï G and Bravo E 2008a Astrophys. J. 680 1149-57

Badenes C, Bravo E and Hughes J P 2008b Astrophys. J. 680 L33-L36

Badenes C, Harris J, Zaritsky D and Prieto J L 2009 Astrophys. J. $700727-40$

Badnell N R, O’Mullane M G, Summers H P, Altun Z, Bautista M A, Colgan J, Gorczyca T W, Mitnik D M, Pindzola M S and Zatsarinny O 2003 Astron. Astrophys. 406 1151-65 http://amdpp.phys.strath.ac.uk/tamoc/DR/ 
Badnell N R 2006a Astrophys. J. 651 L73-L76

Badnell N R 2006b J. Phys. B: At. Mol. Opt. Phys. 39 4825-52

Badnell N R 2006c Astrophys. J. Suppl. Ser. 167 334-42 http://amdpp.phys.strath.ac.uk/tamoc/RR/

Baer H, Mustafayev A, Profumo S, Belyaev A and Tata X 2005 J. High Energy Phys. JHEP07(2005)065

Bahcall J N, Serenelli A M and Pinsonneault M 2004 Astrophys. J. 614 464-71

Bahcall J N, Basu S, Pinsonneault M and Serenelli A M 2005a Astrophys. J. 618 1049-56

Bahcall J N, Serenelli A M and Basu S 2005b Astrophys. J. 621 L85-8

Bailey J E et al 2001 J. Quant. Spectrosc. Radiat. Transfer 71 157-68

Bailey J E et al 2007 Phys. Rev. Lett. 99265002

Bailey J E, Gochau G A, Mancini R C, Igelsias C A, MacFarlane J J, Golovkin I E, Blancard C, Cosse Ph and Faussurier G 2009 Phys. Plasmas 16058101

Balbus S A and Hawley J F 1991 Astrophys. J. 376 214-22

Balbus S A and Hawley J F 1998 Rev. Mod. Phys. 70 1-53

Baker C A et al 2006 Phys. Rev. Lett. 97131801

Bardayan D W et al 2000 Phys. Rev. C 62055804

Bardayan D W et al 2002 Phys. Rev. Lett. 89262501

Barwick S et al 2006 Phys. Rev. Lett. 96171101

Basu S and Antia M H 2008 Phys. Rep. 457 217-83

Basu S 2010 Astrophys. Space Sci. 328 43-50

Bates D R and Lewis J T 1955 Proc. Phys. Soc. Lond. A 68 173-80

Bauman R P, Porter R L, Ferland G J and MacAdam K B 2005 Astrophys. J. 628 541-4

Bauschlicher C W, Ram R S, Bernath P F, Parsons C G and Galehouse D 2001 J. Chem. Phys. 115 1312-18

Bauschlicher C W, Boersma C, Ricca A, Mattioda A L, Cami J, Peeters E, Sánchez de Armas F, Puerta Saborido G, Hudgins D M and Allamandola L J 2010 Astrophys. J. Suppl. Ser. $189341-5$

Bean J, Kempton E M-R and Homeier D 2010 Nature 468 669-72

Behar E, Sako M and Kahn S M 2001 Astrophys. J. 563 497-504

Behar E, Rasmussen A P, Blustin A J, Sako M, Kahn S M, Kaastra J S, Branduardi-Raymont G and Steenbrugge K C 2003 Astrophys. J. 598 232-41

Beiersdorfer P 2003 Ann. Rev. Astron. Astrophys. 41 343-90

Beiersdorfer P, Olson R E, Brown G V, Harris C L, Neill P A, Schweikhard L, Utter S B and Widmann K 2000 Phys. Rev. Lett. 85 5090-3

Beiersdorfer P et al 2003 Science 300 1558-60

Bekooy J P, Verhoeve P, Meerts W L and Dymanus A 1985 J. Chem. Phys. 82 3868-9

Bellan P M 2005 Phys. Plasmas 12058301

Bellan P M, You S and Hsu S C 2005 Astrophys. Space Sci. $298203-9$

Bemmerer D et al 2006a Phys. Rev. Lett. 97122502

Bemmerer D et al 2006b Nucl. Phys. A 779 297-317

Bemmerer D et al 2009 J. Phys. G: Nucl. Part Phys. 36045202

Benjamin R A, Skillman E D and Smits D P 1999 Astrophys. J. $514307-24$

Bergerson W F, Forest C B, Fiksel G, Hannum D A, Kendrick R, Sarff J S and Stambler S 2006 Phys. Rev. Lett. 96015004

Bernabei R et al 2010 Eur. Phys. J. C 67 39-49

Bernstein M P, Dworkin J P, Sandford S A, Cooper G W and Allamandola L J 2002 Nature 416 401-3

Bhardwaj A et al 2007 Planet. Space Sci. 55 1135-89

Biémont E, Palmeri P, Quinet P, Zhang Z G and Svanberg S 2002 Astrophys. J. 567 1276-83

Blandford R et al 2010a New Worlds, New Horizons in Astronomy and Astrophysics (Washington, DC: National Academies Press)

Blandford R et al 2010b Panel Reports-New Worlds, New Horizons in Astronomy and Astrophysics (Washington, DC: National Academies Press)
Blasi P 2009 Phys. Rev. Lett. 103051104

Blondin J M, Fryxell B A and Konigl A 1990 Astrophys. J. $360370-86$

Blustein A J, Branduardi-Raymont G, Behar E, Kaastra J S, Kahn S M, Page M J, Sako M and Steenbrugge K C 2002 Astron. Astrophys. 392 453-67

Bockelée-Morvan D, Crovisier J, Mumma M J and Weaver H A 2004 COMETS II ed M Festou et al (Tuscon, AZ: University of Arizona Press) pp 391-423

Bolton S 2006 36th COSPAR Scientific Assembly, COSPAR, Plenary Meeting (Beijing) vol 36, pp 3775

Bonetti R et al 1999 Phys. Rev. Lett. 82 5205-8

Borucki W J et al 2010 Science 327 977-80

Bouquet S et al 2004 Phys. Rev. Lett. 92225001

Bouwman J, Meeus G, de Koter A, Hony S, Dominik C, Waters L B F M 2001 Astron. Astrophys. 375 950-62

Bouwman J, Cuppen H M, Bakker A, Allamandola L J and Linnartz H 2010 Astron. Astrophys. 511 A33

Bozier J C, Thiell G, Le-Breton J P, Azra S, Decroisette M and Schirmann D 1986 Phys. Rev. Lett. 57 1304-7

Bradley J 2010 Astromineralogy (Lecture Notes in Physics vol 815) ed T Henning (Berlin: Springer) pp 259-76

Bregman J N 2007 Annu. Rev. Astron. Astrophys. 45 221-59

Brickhouse N S and Schmelz J T 2006 Astrophys. J. 636 L53-L56

Brickhouse N S et al 2009a arXiv:0902.4666

Brickhouse N S et al 2009b arXiv:0902.4681

Brickhouse N S et al 2009c arXiv:0902.4688

Brickhouse N S et al 2009d arXiv:0902.4747

Brickhouse N S et al 2009e arXiv:0902.4882

Brickhouse N S, Cranmer S R, Dupree A K, Luna G J M and Wolk S 2010 Astrophys. J. 710 1835-47

Broggini C, Bemmerer D, Guglielmetti A and Menegazzo R 2010 Annu. Rev. Nucl. Part. Sci. 6053

Bronson-Messer O E, Hix W R, Liebendörfer M and Mezzacappa A 2003 Nucl. Phys. A 718 449-51

Brown G V, Beiersdorfer P, Liedahl D A, Widmann K and Kahn S M 1998 Astrophys. J. 502 1015-26

Brown G V, Beiersdorfer P, Liedahl D A, Widmann K and Kahn S M 2000 Astrophys. J. 5321245 (erratum)

Brown G V, Beiersdorfer P, Chen H, Chen M H and Reed K J 2001 Astrophys. J. 557 L75-L78

Brown J M and Müller H S P 2009 J. Mol. Spectrosc. 255 68-71

Brown M R, Cothran C D, Landreman M, Schlossberg D and Matthaeus W 2002 Astrophys. J. 577 L63-L66

Brown T A D, Bordeanu C, Snover K A, Storm D W, Melconian D, Sallaska A L, Sjue S K L and Triambak S 2007 Phys. Rev. C 76055801

Brownlee et al 2006 Science 314 1711-16

Bruhns H et al 2010a Rev. Sci. Instrum. 81013112

Bruhns H, Kreckel H, Miller K A, Urbain X and Savin D W 2010b Phys. Rev. A 82042708

Brünken S, Gupta H, Gottlieb C A, McCarthy M C and Thaddeus P 2007 Astrophys. J. 664 L43-L46

Buckley M R and Randall L 2010 arXiv: 1009.0270

Burrows A, Ram R S, Bernath P, Sharp C M and Milsom J A 2002 Astrophys. J. 577 986-92

Burrows A, Dulick M, Bauschlicher C W, Bernath P F, Ram R S, Sharp C M and Milsom J A 2005 Astrophys. J. 624 988-1002

Busquet M et al 2007 High Energy Density Phys. 3 8-11

Busso M, Gallino R and Wasserburg G J 1999 Annu. Rev. Astron. Astrophys. 37 239-309

Byun D-Y, Koo B-C, Tatematsu K and Sunada K 2006 Astrophys. J. 637 283-95

Calder A et al 2002 Astrophys. J. 143 201-29

Calvet N and Gullbring E 1998 Astrophys. J. 509 802-18

Calzavara A J and Matzner C D 2004 Mon. Not. R. Astron. Soc. $351694-706$ 
Cami J, Bernard-Salas J, Peeters E and Malek S E 2010 Science 329 1180-2

Canto J, Tenorio-Tagle G and Rozyczka M 1988 Astron. Astrophys. 192 287-94

Carena M, Nardini G, Quiros M and Wagner C E M 2009 Nucl. Phys. B 812 243-63

Cartledge S I B, Meyer D M and Lauroesch J T 2003 Astrophys. J. 597 408-13

Cartledge S I B, Lauroesch J T, Meyer D M and Sofia U J 2006 Astrophys. J. 641 327-46

Castelli F, Gratton R G and Kurucz R L 1997 Astron. Astrophys. 318 841-69

Castelli F and Kurucz R L 2004 Astron. Astrophys. 419 725-33

CDMS II Collaboration 2010 Science 327 1619-21

Cernicharo J, Guélin M, Agúndez M, Kawaguchi K, McCarthy M and Thaddeus P 2007 Astron. Astrophys. 467 L37-L40

Chafa A et al 2007 Phys. Rev. C 75035810

Chakraborty N, Fields B D and Olive K A 2010 Phys. Rev. D 83063006

Chakravorty S, Kembhavi A K, Elvis M, Ferland G and Badnell N R 2008 Mon. Not. R. Astron. Soc. 384 L24-L28

Chakravorty S, Kembhavi A K, Elvis M, Ferland G and Badnell N R 2009 Mon. Not. R. Astron. Soc. 393 83-98

Charbonneau D et al 2005 Astrophys. J. 626 523-9

Charbonneau D, Knutson H A, Barman T, Allen L E, Mayor M, Megeath S T, Queloz D and Udry S 2008 Astrophys. J. 686 1341-8

Charbonnel C, Tosi M, Primas and Chiappini C 2010 Light Elements in the Universe (Proc. IAU Symp. vol 268) (Cambridge: Cambridge University Press)

Chastaing D, Le Picard S D, Sims I R and Smith I W M 2001 Astron. Astrophys. 365 241-7

Chen D, Gao H and Kwong V H 2003 Phys. Rev. A 68052703

Chen G-X 2008 Mon. Not. R. Astron. Soc. 386 L62-L66

Chen J-W and Savage M J 1999 Phys. Rev. C 60065205

Chen G-X, Smith R K, Kirby K, Brickhouse N S and Wargelin B J 2006 Phys. Rev. A 74042709

Chevalier R A 1992 Nature 355 691-6

Chevalier R A and Fransson C 2008 Astrophys. J. 683 L135-L138

Chevalier R A and Imamura J N 1982 Astrophys. J. 261 543-9

Chiar J E and Tielens A G G M 2006 Astrophys. J. 637 774-85

Chipps K A et al 2009 Phys. Rev. Lett. 102152502

Chowdhury P K, Merer A J, Rixon S J Bernath P F and Ram R S 2005 Phys. Chem. Chem. Phys. 8 822-6

Christensen-Dalsgaard J, di Mauro M P, Houdek G and Pijpers F 2009 Astron. Astrophys. $494205-8$

Chugai N N and Chevalier R A 2006 Astrophys. J. 641 1051-9

Ciardi A, Lebedev S V, Frank A, Suzuki-Vidal, F, Hall G N, Bland S N, Harvey-Thompson A, Blackman E G and Camenzind M 2009 Astrophys. J. 691 L147-L150

Cirigliano V, Profumo S and Ramsey-Musolf M J 2006 J. High Energy Phys. JHEP07(2006)002

Cirigliano V, Li Y, Profumo S and Ramsey-Musolf M J 2010 J. High Energy Phys. JHEP01(2010)002

Clemett S J, Sandford S A, Nakamura-Messenger K, Horz F and McKay D S 2010 Meteorit. Planet. Sci. 45 701-22

Coc A and Vangioni E 2010 J. Phys.: Conf. Ser. 202012001

Cody G D et al 2008 Meteorit. Planet. Sci. 43 353-65

Cohen D, MacFarlane J, Bailey J and Liedahl D 2003 Rev. Sci. Instrum. 74 1962-5

Cohen D H, Leutenegger M A, Wollman E E, Zsargó J, Hillier D J, Townsend R H D and Owocki S P 2010 Mon. Not. R. Astron. Soc. 405 2391-405

Commins E D, Ross S B, DeMille D and Regan B C 1994 Phys. Rev. A $502960-77$

Confortola F et al 2007 Phys. Rev. C 75065803

Cordiner M and Millar T J 2009 Astrophys. J. 697 68-78

Costantini H, Formicola A, Imbriani G, Junker M, Rolfs C and Strieder F 2009 Rep. Prog. Phys. 72086301
Cothran C D, Brown M R, Grat T, Schaffer M J and Marklin G 2009 Phys. Rev. Lett. 103215002

Cowan J J and Sneden C 2006 Nature 440 1151-6

Cowan J J and Thielemann F-K 2004 Phys. Today 57 47-53

Cranmer S R, Panasyuk A V and Kohl J L 2008 Astrophys. J. 678 1480-97

Cravens T E 1997 Geophys. Res. Lett. 24 105-8

Cravens T E, Robertson I P and Snowden S L 2001 J. Geophys. Res. 106 24883-92

Crespo Lopez-Urrutia J R, Neger T and Jager H 1994 J. Phys. D: Appl. Phys. 27 994-8

Crovisier J, Leech K, Bockelee-Morvan D, Brooke T Y, Hanner M S, Altieri B, Keller H U and Lellouch E 1997 Science 275 1904-7

Crovisier J et al 2004 Astron. Astrophys. 418 1141-57

Curry J J, den Hartog E A and Lawler J E 1997 J. Opt. Soc. Am. B $142788-99$

Dalgarno A 1976 Atomic Processes and Applications ed P G Burke (Amsterdam: North-Holland) p 109

Davoudias H, Morrissey D E, Sigurdson K and Tulin S 2010 Phys. Rev. Lett. 105211304

De Vries M S, Reihs K, Wendt H R, Golden W G, Hunziker H E, Fleming R, Peterson E and Chang S 1993 Geochim. Cosmochim. Acta 57 933-8

Deamer D, Dworkin J P, Sandford S A, Bernstein M P and Allamandola L J 2002 Astrobiology 2 371-81

Decrock P et al 1991 Phys. Rev. Lett. 67 808-11

Delbar The et al 1993 Phys. Rev. C 48 3088-96

DeMeo F E, Barucci M A, Merlin F, Guilbert-Lepoutre A, Alvarez-Candal A, Delsanti A, Fornasier S and de Bergh C 2010 Astron. Astrophys. 521 A35

Deming D, Seager S, Richardson L J and Harrington J 2005 Nature $434740-3$

Demorest P B, Pennucci T, Ransom S M, Roberts M S E and Hessels J W T 2010 Nature 467 1081-3

den Hartog E A, Curry J J, Wickliffe M E and Lawler J E 1998 Sol. Phys. 178 239-44

den Hartog E A, Wiese L M and Lawler J E 1999 J. Opt. Soc. Am. B $162278-84$

den Hartog E A, Fedchak J A and Lawler J E 2001 J. Opt. Soc. Am. B 18 861-5

den Hartog E A, Wickliffe M E and Lawler J E 2002 Astrophys. J. Suppl. Ser. 141 255-65

den Hartog E A, Lawler J E, Sneden C and Cowan J J 2003 Astrophys. J. Suppl. Ser. 148 543-66

den Hartog E A, Herd M T, Lawler J E, Sneden C, Cowan J J and Beers T C 2005 Astrophys. J. 619 639-55

den Hartog E A, Lawler J E, Sneden C and Cowan J J 2006 Astrophys. J. Suppl. Ser. 167 292-314

den Hartog E A, Lawler J E, Sobeck J S, Sneden C and Cowan J J 2011 Astrophys. J. Suppl. Ser. 19435

Dennerl K 2010 Space Sci. Rev. 157 57-91

d'Enterria D, Engel R, Pierog T, Ostapchenko S and Werner K 2011 Astropart. Phys. 35 98-113

di Leva A et al 2009 Phys. Rev. Lett. 102232502

Diemand J, Kuhlen M and Madau P 2007 Astrophys. J. 657 262-70

Dine M and Kusenko A 2003 Rev. Mod. Phys. 76 1-30

Dobler G, Finkbeiner D P, Cholis I, Slatyer T and Weiner N 2010 Astrophys. J. 717 825-842

Doron R and Behar E 2002 Astrophys. J. 574 518-26

Dorschner J and Henning T 1995 Astron. Astrophys. Rev. 6 271-333

Dos Santos S F, Kokoouline V and Greene C H 2007 J. Chem. Phys. 127124309

Doss F W 2011 PhD Thesis University of Michigan

Doss F W, Robey H F, Drake R P and Kuranz C C 2009 Phys. Plasmas 16112705

Doss F W, Drake R P and Kuranz C C 2010 High Energy Density Phys. 6 157-61

Draine B T 2003 Annu. Rev. Astron. Astrophys. 41 241-89

Drake R P 1999 J. Geophys. Res. 104 14505-15 
Drake J J, Ratzlaff P W, Laming J M and Raymond J 2009 Astrophys. J. 703 1224-9

Duffy L D and van Bibber K 2009 New J. Phys. 11105008

Duffy A R, Schaye J, Kay S T, Dalla Vecchia C, Battye R A and Booth C M 2010 Mon. Not. R. Astron. Soc. 405 2161-78

Dworkin L P, Deamer D W, Sandford S A and Allamandola L J 2001 Proc. Natl Acad. Sci. USA 98 815-19

Edens A D, Ditmire T, Hansen J F, Edwards M J, Adams R G, Rambo P K, Ruggles L, Smith I C and Porter J L 2005 Phys. Rev. Lett. 95244503

Edwards M J, MacKinnon A J, Zweiback J, Shigemori K, Ryutov D D, Rubenchik A M, Keitlty K A, Liang E, Remington B A and Ditmire T 2001 Phys. Rev. Lett. 87085004

Eggert J, Brygoo S, Loubeyre P, McWilliams R S, Celliers P M, Hicks D G, Boehly T R, Jeanloz R and Collins G W 2008 Phys. Rev. Lett. 100124503

Elsila J E, Dworkin J P, Bernstein M P, Martin M P and Sandford S A 2007 Astrophys. J. 660 911-18

Elsila J E, Glavin D P and Dworkin J P 2009 Meteorit. Planet. Sci. 44 1323-30

Emery J P, Cruikshank D P and van Cleve J 2006 Icarus 182 496-512

Epp S W et al 2007 Phys. Rev. Lett. 98183001

Fabian J 1996 Phys. Rev. B 53 13864-70

Falgarone E et al 2010 Astron. Astrophys. 521 L15

Falize E, Bouquet S and Michaut C 2009a Astrophys. Space Sci. 322 107-11

Falize E, Michaut C, Cavet C, Bouquet S, Koenig M, Loupias B, Ravasio A and Gregory C G 2009b Astrophys. Space Sci. $32271-5$

Falize E et al 2011 Astrophys. Space Sci. 726 41-9

Farley D R, Estabrook K G, Glendinning S G, Glenzer S H, Remington B A, Shigemori K, Stone J M, Wallace R J, Zimmerman G B and Harte J A 1999 Phys. Rev. Lett. 83 1982-5

Fedchak J A, den Hartog E A, Lawler J E, Palmeri P, Quinet P and Biémont E 2000 Astrophys. J. 542 1109-18

Federman S R, Knauth D C and Lambert D L 2004 Astrophys. J. 603 L105-L108

Ferland G F, Korista K T, Verner D A, Ferguson J W, Kingdon J B and Verner E M 1998 Publ. Astron. Soc. Pac. 110 761-78

Fesen R A, Zastrow J A, Hammell M C, Shull J M and Silvia D W 2011 Astrophys. J. 736109

Field G B, Goldsmith D W and Habing H J 1969 Astrophys. J. 155 L149

Fiksel G, Almagri A F, Chapman B E, Mirnov V V, Ren Y, Sarff J S and Terry P W 2009 Phys. Rev. Lett. 103145002

Fink U 2009 Icarus 201 311-34

Fitzpatrick A L, Hooper D and Zurek K M 2010 Phys. Rev. D 81115005

Flynn et al 2006 Science 314 1731-5

Foord M E et al 2004 Phys. Rev. Lett. 93055002

Foord M E et al 2006 J. Quant. Spectrosc. Radiat. Transfer $99712-29$

Formicola A et al 2004 Phys. Lett. B 591 61-8

Fortney J J and Nettelmann N 2010 Space Sci. Rev. 152 423-47

Fortney J J, Glenzer S H, Koenig M, Militzer B, Saumon D and Valencia D 2009 Phys. Plasmas 16041003

Foster J M et al 2005 Astrophys. J. 634 L77-L80

Fox C, Iliadis C, Champagne A E, Fitzgerald R P, Longland R, Newton J, Pollanen J and Runkle R 2005 Phys. Rev. C 71055801

Fox P J and Poppitz E 2009 Phys. Rev. D 79083528

Fragile P C, Murray S D, Anninos P and van Breugel W 2004 Astrophys. J. $\mathbf{6 0 4} 74-87$

Frum C I, Engleman R Jr, Hedderich H G, Bernath P F, Lamb L D and Huffman D R 1991 Chem. Phys. Lett. 176 504-8

Fujioka et al 2009 Nature Phys. 5 821-5

Fukugita M and Yanagida T 1986 Phys. Lett. B 174 45-7
Fullerton A W, Massa D L and Prinja R K 2006 Astrophys. J. 637 1025-39

Fussen D and Kubach C 1986 J. Phys. B: At. Mol. Opt. Phys. 19 L31-L34

Gailitis A et al 2000 Phys. Rev. Lett. 84 4365-8

Gallo L C, Boller Th, Brandt W N, Fabian A C and Vaughan S 2004 Astron. Astrophys. 417 29-38

Gao H and Kwong V H 2003 Phys. Rev. A 68052704

García-Hernández D A, Manchado A, García-Lario P, Stanghellini L, Villaver E, Shaw R A, Szczerba R and Perea-Calderón J V 2010 Astrophys. J. 724 L39-L43

Garg U et al 2007 Nucl. Phys. A 788 36-43

Garrod R T and Herbst E 2006 Astron. Astrophys. 457 927-36

Geppert W D and Larsson M 2008 Mol. Phys. 106 2199-226

Gerbier G 2010 arXiv: 1012.2260

Gibb E L, Whittet D C B, Boogert A C A and Tielens A 2004 Astrophys. J. Suppl. Ser. 151 35-73

Gillaspy J D, Lin T, Tedesco L, Tan J N, Pomeroy J M, Laming J M, Brickhouse N, Chen G-X and Silver E 2011 Astrophys. J. 728 132-43

Gillett F C, Forrest W J and Merrill K M 1973 Astrophys. J. 183 87-93

Giudice G F, Notari A, Raidal M and Strumia A 2004 Nucl. Phys. B 685 89-149

Glosík J, Korolov I, Plašil R, Novotný O, Kotrık T, Hlavenka P, Varju J, Mikhailov I A, Kokoouline V and Greene C H 2008 J. Phys. B: At. Mol. Opt. Phys. 41191001

Glosík J, Plašil R, Korolov I, Kotrík T, Novotný O, Hlavenka P, Dohnal P, Varju J, Kokoouline V and Greene C H 2009 Phys. Rev. A 79052707

Glover S C, Savin D W and Jappsen A-K 2006 Astrophys. J. $640553-68$

Glover S C O and Abel T 2008 Mon. Not. R. Astron. Soc. 388 1627-51

Goodman M W and Witten E 1985 Phys. Rev. D 31 3059-63

Gorham P W, Saltzberg D, Field R C, Guillian E, Milinčić R, Miočinovič P, Walz D and Williams D 2005 Phys. Rev. D 72023002

Greenwood J B, Williams I D, Smith S J and Chutjian A 2000 Phys. Rev. A 63062707

Greisen K 1966 Phys. Rev. Lett. 16 748-50

Griffith W C, Swallows M D, Loftus T H, Romalis M V, Heckel B R, Fortson E N 2009 Phys. Rev. Lett. 102101601

Grillmair C J, Burrows A, Charbonneau D, Armus L, Stauffer J, Meadows V, van Cleve J, von Braun K and Levine D 2008 Nature 456 767-9

Grosdidier Y, Moffat A F J, Joncas G and Acker A 1998 Astrophys. J. 506 L127-L131

Grosskopf M J, Drake R P, Kuranz C C, Miles A R, Hansen J F, Plewa T, Hearn N, Arnett D and Wheeler J C 2009 Astrophys. Space Sci. 322 57-63

Grun J, Stamper J, Manka C, Resnick J, Burris R, Crawford J and Ripin B H 1991 Phys. Rev. Lett. 66 2738-41

Grundy W M and Schmitt B 1998 J. Geophys. Res. 103 25809-22

Grundy W M, Young L A, Spencer J R, Johnson R E, Young E F and Buie M W 2006 Icarus 184 543-55

Grupen C 2005 Astroparticle Physics (Berlin: Springer)

Gu M F 2004 Astrophys. J. Suppl. Ser. 153 389-93

Gu M F, Holczer T, Behar E and Kahn S M 2006 Astrophys. J. 641 1227-32

Gudipati M S 2004 J. Phys. Chem. A 108 4412-9

Gudipati M S and Allamandola L J 2004 Astrophys. J. 615 L177-L180

Gudipati M S and Allamandola L J 2006 J. Phys. Chem. A $1109020-4$

Gudipati M S et al 2009 arXiv:0910.0442

Gunther H M, Lewandowska N, Hundertmark M P G, Steinle H, Schmitt J, Buckley D, Crawford S, O'Donoghue D and Vaisanen P 2010 Astron. Astrophys. 518 A54 
Guo B and Li Z H 2007 Chin. Phys. Lett. 24 65-8

Gupta $\mathrm{H}$ et al 2010 Astron. Astrophys. 521 L47

Guzman J and Plewa T 2009 Nonlinearity 22 2775-97

Gyurky G et al 2007 Phys. Rev. C 75035805

Halfen D T and Ziurys L M 2004 Astrophys. J. 611 L65-L68

Halfen D T, Sun M, Clouthier D J and Ziurys L M 2009 J. Chem. Phys. 130014305

Hammache F et al 1998 Phys. Rev. Lett. 80928

Hammache F et al 2001 Phys. Rev. Lett. 863985

Hanner M S and Zolensky M E 2010 Astromineralogy (Lecture Notes in Physics vol 815) ed T Henning (Berlin: Springer) pp 275-315

Hansen J F, Edwards M J, Froula D H, Edens A D, Gregori G and Ditmire T 2006 Phys. Plasmas 13112101

Harada N, Herbst E and Wakelam V 2010 Astrophys. J. 721 1570-8

Harrison J J, Brown J M, Halfen D T and Ziurys L M 2006 Astrophys. J. 637 1143-7

Hartigan P and Morse J 2007 Astrophys. J. 660 426-40

Hartigan P, Foster J M, Wilde B H, Coker R F, Rosen P A, Hansen J F, Blue B E, Williams R J R, Carver R and Frank A 2009 Astrophys. J. 705 1073-94

Hartigan P, Frank A, Foster J M, Wilde B H, Douglas M, Rosen P A, Coker R F, Blue B E and Hansen J F 2011 Astrophys. J. 73629

Hauschildt P, Warmbier R, Schneider R and Barman T 2009 Astron. Astrophys. 504 225-9

Haxton W C and Serenelli A M 2008 Astrophys. J. 687 678-91

Heger A, Langanke K, Martinez-Pinedo G and Woosley S E 2001 Phys. Rev. Lett. 86 1678-81

Heger A, Kolbe E, Haxton W C, Langanke K, Martínez-Pinedo G and Woosley S E 2005 Phys. Lett. B 606 258-64

Heil M, Käppeler F, Uberseder E, Gallino R, Bisterzo S and Pignatari M 2008 Phys. Rev. C 78025802

Henning T 2010 Annu. Rev. Astron. Astrophys. 48 21-46

Henning T and Salama F 1998 Science 282 2204-10

Henry R B C, Cowan J J and Sobeck J S 2010 Astrophys. J. $709715-24$

Herbst E 1981 Nature 289 656-7

Herbst E and Klemperer W 1973 Astrophys. J. 185 505-34

Herbst E and van Dishoeck E F 2009 Annu. Rev. Astron. Astrophys. $47427-80$

Hernanz M, José J, Coc A, Gómez-Gomar J and Isern J 1999 Astrophys. J. $\mathbf{5 2 6}$ L97-L100

Heros C d 12010 arXiv:1012.0184

Hersant F, Wakelam V, Dutrey A, Guilloteau S and Herbst E 2009 Astron. Astrophys. 493 L49-L52

Hessels J W T, Ransom S M, Stairs I H, Freire P C C, Kaspi V M and Camilo F 2006 Science 311 1901-4

Hicks D G, Boehly T R, Celliers P M, Eggert J H, Moon S J, Meyerhofer D D and Collins G W 2009 Phys. Rev. B 79014112

Hohenberger M et al 2010 Phys. Rev. Lett. 105205003

Hooper D, Finkbeiner D P and Dobler G 2007 Phys. Rev. D 76083012

Hora J L, Latter W B, Smith H A and Marengo M 2006 Astrophys. J. 652 426-41

Horn A, Mollendal H, Sekiguchi O, Uggerud E, Roberts H, Herbst E, Viggiano A A and Fridgen T D 2004 Astrophys. J. $611605-14$

Hörz et al 2006 Science 314 1716-9

Hovde D C and Saykally R J 1987 J. Chem. Phys. 87 4332-8

Huet P and Nelson A E 1996 Phys. Rev. D 53 4578-97

Hwang U, Flanagan K A and Petre R 2005 Astrophys. J. $635355-64$

IAU Commission $142011 \mathrm{http}: / /$ iacs.cua.edu/IAUC14/

Iglesias-Groth S, Manchado A, García-Hernández D A, González-Hernández J I and Lambert D L 2008 Astrophys. J. 685 L55-L58

Iglesias-Groth S, Manchado A, Rebolo R, González-Hernández J I, García-Hernández D A and Lambert D L 2010 Mon. Not. R. Astron. Soc. 407 2157-65
Iglesias-Groth S, Manchado A, Rebolo R, González-Hernández J I, García-Hernández D A and Lambert D L 2010 Mon. Not. R. Astron. Soc. 409880 (erratum)

Imbriani G et al 2005 Eur. Phys. J. A 25 455-66

Indriolo N, Geballe T R, Oka T and McCall B J 2007 Astrophys. J. $6711736-47$

Indriolo N, Fields B and McCall B J 2009 Astrophys. J. $694257-67$

Innes D E, Giddings J R and Falle S A E G 1987 Mon. Not. R. Astron. Soc. 226 67-93

Ivarsson S, Litzén U and Wahlgren G M 2001 Phys. Scr. 64 455-461

Ivarsson S, Andersen J, Nordström B, Dai X, Johansson S, Lundberg $\mathrm{H}$, Nilsson $\mathrm{H}$, Hill $\mathrm{V}$, Lundqvist $\mathrm{M}$ and Wyart J F 2003 Astron. Astrophys. 409 1141-9

Izotov Y I and Thuan T X 2010 Astrophys. J. 710 L67-L71

Jager C, Huisken F, Mutschke H, Henning Th, Poppitz W and Voicu I 2007 Carbon 47 2981-94

Jewitt D 1999 Annu. Rev. Earth Planet. Sci. 27 287-312

Jewitt D C and Luu J 2004 Nature 432 731-3

Ji H, Burin M, Schartman E and Goodman J 2006 Nature 444 343-6

Juhasz A, Henning Th, Bouwman J, Dullemond C P, Pascucci I, Apai D 2009 Astrophys. J. 695 1024-41

Julien K and Knobloch E 2010 Phil. Trans. R. Soc. A 368 1607-33

Junghans A R, Mohrmann E C, Snover K A, Steiger T D, Adelberger E G, Casandjian J M, Swanson H E, Buchmann L, Park S H and Zyuzin A 2002 Phys. Rev. Lett. 88041101

Junghans A R et al 2003 Phys. Rev. C 68065803

Junghans A R, Snover K A, Mohrmann E C, Adelberger E G and Buchmann L 2010 Phys. Rev. C 81012801

Käppeler F, Beer H and Wisshak K 1989 Rep. Prog. Phys. 52 945-1013

Käppeler F, Gallino R, Bisterzo S and Aoki W 2011 Rev. Mod. Phys. 83 157-93

Kallman T R 2010 Space Sci. Rev. 157 177-91

Kallman T R and Palmeri P 2007 Rev. Mod. Phys. 79 79-133

Kaltenegger L and Sasselov D 2010 Astrophys. J. 708 1162-7

Kalvans J and Shmeld I 2010 Astron. Astrophys. 521 A37

Kang Y G et al 2000 Proc. SPIE 3886 489-95

Kaspi S et al 2002 Astrophys. J. 574 643-62

Kaspi S, Netzer H, Chelouche D, George I M, Nandra K and Turner T J 2004 Astrophys. J. 611 68-80

Kastner J H, Huenemoerder D P, Schulz N S, Canizares C R and Weintraub D A 2002 Astrophys. J. 567 434-40

Kawaguchi K, Kasai Y, Ishikawa S and Kaifu N 1995 Publ. Astron. Soc. Japan 47 853-76

Keller et al 2006 Science 314 1728-31

Kelley M S and Wooden D H 2009 Planet. Space Sci. 57 1133-45

Kharchenko V and Dalgarno A 2000 J. Geophys. Res. 105 18351-60

Kifonidis K, Plewa T, Janka H-T and Muller E 2000 Astrophys. J. 531 L123-L126

Kifonidis K, Plewa T, Janka H-T and Muller E 2003 Astron. Astrophys. 408 621-49

Kifonidis K, Plewa T, Sheck L, Janka H-T and Muller E 2006 Astron. Astrophys. 457 963-86

Kim H, Wyrowski F, Menten K M, Decin L 2010 Astron. Astrophys. 516 A68

Kimoto P A and Chernoff D F 1997 Astrophys. J. 485 274-84

Kirkpatrick J D 2005 Annu. Rev. Astron. Astrophys. 43 195-245

Klein R I, McKee C F and Colella P 1994 Astrophys. J. $420213-36$

Klein R I, Budil K S, Perry T S and Bach D R 2003 Astrophys. J. $\mathbf{5 8 3} 245-59$

Klypin A, Trujillo-Gomez S and Primack J 2011 Astrophys. J. 740102

Knutson H A, Charbonneau D, Allen L E, Burrows A and Megeath S T 2008 Astrophys. J. 673 526-31

Knutson H A, Charbonneau D, Allen L E, Fortney J J, Agol E, Cowan N B, Showman A P, Cooper C S and Megeath S T 2007 Nature 447 183-6 
Kobayashi K et al 2005 Phys. Rev. D 72052007

Koenig M et al 2006 Phys. Plasmas 13056504

Kohl J L, Noci G, Cranmer S R and Raymond J L 2006 Astron. Astrophys. Rev. 13 31-157

Koldoba A V, Ustyugova G V, Romanova M M and Lovelace R V E 2008 Mon. Not. R. Astron. Soc. 388 357-66

Konacki M, Torres G, Jha S and Sasselov D D 2003 Nature 421 507-9

Konacki M, Torres G, Sasselov D D, Pietrzyński G, Udalski A, Jha S, Ruiz M T, Gieren W and Minniti D 2004 Astrophys. J. 609 L37-L40

Konigl A 1991 Astrophys. J. 370 L39-L43

Kotera K and Olinto A V 2011 Annu. Rev. Astron. Astrophys. 49 119-53

Kotrík T, Dohnal P, Korolov I, Plašil R, Roučka Š, Glosík J, Greene C H and Kokoouline V 2010 J. Chem. Phys. 133034305

Koutroumpa D, Lallement R, Kharchenko V, Dalgarno A, Pepino R, Izmodenov V and Quemerais E 2006 Astron. Astrophys. 460 289-300

Kraemer S B, Ferland G J and Gabel J R 2004 Astrophys. J. $604556-61$

Krätschmer W, Lamb L D, Fostiropoulos K and Huffman D R 1990 Nature 347 354-8

Krasnopolsky V A and Mumma M J 2001 Astrophys. J. 549 634-4

Krasnopolsky V A, Mumma M J, Abbott M, Flynn B C, Meech K J, Yeomans D K, Feldman P D and Cosmovici C B 1997 Science 277 1488-91

Kratz K-L, Pfeiffer B, Thielemann F-K and Walters W B 2000 Hyperfine Interact. 129 185-221

Krause O, Tanaka M, Usuda T, Hattori T, Goto M, Birkmann S and Nomoto K 2008 Nature 456 617-19

Kravchencko I et al 2006 Phys. Rev. D 73082002

Kreckel H et al 2005 Phys. Rev. Lett. 95263201

Kreckel H et al 2010 Phys. Rev. A 82042715

Kreckel H, Bruhns H, Čížek M, Glover S C O, Miller K A, Urbain X and Savin D W 2010 Science 329 69-71

Krełowski J, Beletsky Y, Galazutdinov G A, Kołos R, Gronowski M and LoCurto G 2010 Astrophys. J. 714 L64-L67

Krolik J, McKee C M and Tarter C B 1981 Astrophys. J. 249 422-42

Krongold Y, Nicastro F, Brickhouse N S, Elvis M, Liedahl D A and Mathur S 2003 Astrophys. J. 597 832-50

Krongold Y, Nicastro F, Elvis M, Brickhouse N S, Mathur S and Zezas A 2005 Astrophys. J. 620 165-82

Kroto H W, Heath J R, Obrien S C, Curl R F and Smalley, R E 1985 Nature 318 162-3

Kuhlen M, Weiner N, Diemand J, Madau P, Moore B, Potter D, Stadel J and Zemp M 2010 J. Cosmol. Astropart. Phys. JCAP02(2010)030

Kuranz C C et al 2009 Astrophys. J. 696 749-59

Kuranz C C, Drake R P, Grosskopf M J, Fryxell B, Budde A, Hansen J F, Miles A R, Plewa T, Hearn N C and Knauer J P 2010 Phys. Plasmas 17052709

Kurucz R L and Bell B 1995 Kurucz CD-ROM 23: Atomic Line List (Cambridge, MA: Smithsonian Astrophysical Observatory)

Kuzmin V A, Rubakov V A and Shaposhnikov M E 1985 Phys. Lett. B $15536-42$

Laming J M 2004 Phys. Rev. E 70057402

Laming J M et al 2000 Astrophys. J. 545 L161-L164

Landi E and Cranmer S R 2009 Astrophys. J. 691 794-805

Langanke K and Martinez-Pinedo G 2003 Rev. Mod. Phys. 75 819-62

Lattanzi V, Walters A, Drouin B J, Pearson J C 2007 Astrophys. J. $662771-8$

Lawler J E, Bonvallet G and Sneden C 2001a Astrophys. J. $556452-60$

Lawler J E, Wickliffe M E, Cowley C R and Sneden C 2001b Astrophys. J. Suppl. Ser. 137 341-9

Lawler J E, Wickliffe M E, den Hartog E A and Sneden C 2001c Astrophys. J. 563 1075-88
Lawler J E, Sneden C and Cowan J J 2004 Astrophys. J. 604 850-60

Lawler J E, den Hartog E A, Labby Z E, Sneden C, Cowan J J and Ivans I I 2007 Astrophys. J. Suppl. Ser. 169 120-36

Lawler J E, den Hartog E A, Sneden C and Cowan J J 2008a Can. J. Phys. 86 1033-8

Lawler J E, Sneden C, Cowan J J, Wyart J-F, Ivans I I, Sobeck J S, Stockett M H and den Hartog E A 2008b Astrophys. J. 178 71-88

Lawler J E, Sneden C, Cowan J J, Ivans I I and den Hartog E A 2009 Astrophys. J. Suppl. Ser. 182 51-79

Lebedev S V et al 2002 Astrophys. J. 564 113-19

Lebedev S V, Ampleford D, Ciardi A, Bland S N, Chittenden J P, Haines M G, Frank A, Blackman E G and Cunningham A 2004 Astrophys. J. 616 988-97

Lebedev S V et al 2005 Mon. Not. R. Astron. Soc. 361 97-108

Lee C, Cirigliano V and Ramsey-Musolf M J 2005 Phys. Rev. D 71075010

Leith C E 1990 Phys. Fluids A 2 297-9

Lemut A et al 2006 Phys. Lett. B 634 483-7

Leonard D S, Karwowski H J, Brune C R, Fisher B M and Ludwig E J 2006 Phys. Rev. C 73045801

Lestinsky M et al 2009 Astrophys. J. 698 648-59

Li Y, Profumo S and Ramsey-Musolf M 2008 Phys. Rev. D 78075009

Li Y, Profumo S and Ramsey-Musolf M 2009 Phys. Lett. B 673 95-100

Li Y, Profumo S and Ramsey-Musolf M 2010 J. High Energy Phys. JHEP08(2010)062

Li Z H et al 2006 Phys. Rev. C 74035801

Lin C-H, Antia H M and Basu S 2007 Astrophys. J. 668 603-10

Lin C-J 2011 arXiv:1101.0261

Linden T and Profumo S 2010 Astrophys. J. 714 L228-L232

Lis D C et al 2010 Astron. Astrophys. 521 L9

Lisse C M et al 1996 Science 274 205-9

Lisse C M, Christian D J, Dennerl K, Meech K J, Petre R, Weaver H A and Wolk S J 2001 Science 292 1343-8

Liszt H S, Lucas R and Pety J 2006 Astron. Astrophys. 448 253-9

Loch S D, Pindzola M S, Ballance C P and Griffin D C 2006 J. Phys. B: At. Mol. Opt. Phys. 39 85-104

Lodders K 2003 Astrophys. J. 591 1220-47

Lodders K and Fegley B Jr 1999 Asymptotic Giant Branch Stars (Proc. IAU Symp. vol 191) ed T le Bertre et al (Cambridge: Cambridge University Press) pp 279-90

Lopez R E and Turner M S 1999 Phys. Rev. D 59103502

Lukić D et al 2007 Astrophys. J. 664 1244-52

Lundberg H, Johansson S, Nilsson H and Zhang Z 2001 Astron. Astrophys. 372 L50-L52

Maercker M, Schoier F L, Olofsson H, Bergman P, Ramstedt S 2008 Astron. Astrophys. 479 779-91

Maier J P, Walker G A H, Bohlender D A, Mazzotti F J, Raghunandan R, Fulura J, Garkusha I and Nagy A 2011 Astrophys. J. $\mathbf{7 2 6} 41$

Malloci G, Joblin C and Mulas G 2007 Chem. Phys. 332 353-9

Maloney P R, Hollenbach D J and Tielens A G G M 1996 Astrophys. J. 466 561-84

Mancini R C, Bailey J E, Hawley J F, Kallman T, Witthoeft M, Rose S J and Takabe H 2009 Phys. Plasmas 16041001

Marta M et al 2008 Phys. Rev. C 78022802

Martin M C, Koller D and Mihaly L 1993 Phys. Rev. B 47 14607-10

Mastrapa R M E and Brown R H 2006 Icarus 183 207-14

Matsumoto C, Leighly K M and Marshall H L 2004 Astrophys. J. 603 456-62

Matthews C N and Minard R D 2006 Faraday Discuss. 133 393-401

Maxted P F L et al 2010 Astron. J. 140 2007-12

McCall B J et al 2003 Nature 422 500-2

McCarthy M C, Gottlieb C A Gupta H and Thaddeus P 2006 Astrophys. J. 652 L141-L144

McClendon J H 1999 Earth-Sci. Rev. 47 71-93 
McKeegan et al 2006 Science 314 1724-8

McKernan B, Yaqoob T and Reynolds C S 2007 Mon. Not. R. Astron. Soc. 379 1359-72

McWilliam A 1997 Annu. Rev. Astron. Astrophys. 35 503-56

Medvedev M V and Loeb A 1999 Astrophys. J. 526 697-706

Menten K M, Wyrowski F, Belloche A, Güsten R, Dedes L and Müller H S P 2011 Astron. Astrophys. 525 A77

Milam S N, Halfen D T, Tenenbaum E D, Apponi A J, Woolf N J and Ziurys L M 2008 Astrophys. J. 684 618-25

Militzer B 2009 Phys. Rev. B 79155105

Miles A R, Braun D G, Edwards M J, Robey H F, Drake R P and Leibrandt D R 2004 Phys. Plasmas 11 3631-45

Miller-Ricci E, Seager S and Sasselov D 2009 Astrophys. J. 690 1056-67

Miyake S, Stancil P C, Sadeghpour H R, Dalgarno A, McLaughlin B M and Forrey R C 2010 Astrophys. J. 709 L168-71

Möller P, Pfeiffer B and Kratz K-L 2003 Phys. Rev. C 67055802

Molster F J and Waters L B F M 2003 Astromineralogy (Lecture Notes in Physics vol 609) ed T Henning (Berlin: Springer) pp 121-70

Moore A S, Gumbrell E T, Lazarus J, Hohenberger M, Robinson J S, Smith R A, Plant T J A, Symes D R and Dunne M 2008 Phys. Rev. Lett. 100055001

Monchaux R et al 2007 Phys. Rev. Lett. 98044502

Morton D C 2000 Astrophys. J. Suppl. Ser. 130 403-36

Morton D C 2003 Astrophys. J. Suppl. Ser. 149 205-38

Moseley J, Aberth W and Peterson J R 1970 Phys. Rev. Lett. 24 435-9

Muller E, Fryxell B and Arnett D 1991 Astron. Astrophys. 251 $505-14$

Müller H S P, Schlöder F, Stutzki J and Winnewisser G 2005 J. Mol. Struct. 742 215-27

Muñoz Caro G M, Meierhenrich U J, Schutte W A, Barbier B, Arcones Segovia A, Rosenbauer H, Thiemann W H-P, Brack A and Greenberg J M 2002 Nature 416 403-6

Murphy A St J et al 2009 Phys. Rev. C 79058801

Murphy G C, Dieckmann M E, Bret A and Drury L O C 2010 Astron. Astrophys. 524 A84

Mürtz P, Zink L R, Evenson K M and Brown J M 1998 J. Chem. Phys. 109 9744-52

Nemes L, Ram R S, Bernath P F, Tinker F A, Zumwalt M C, Lamb L D and Huffman D R 1994 Chem. Phys. Lett. 218 295-303

Netzer H 2004 Astrophys. J. 604 551-5

Netzer H et al 2003 Astrophys. J. 599 933-48

Neufeld D A et al 2010 Astron. Astrophys. 521 L10

Nilsson H, Ivarsson S, Johansson S and Lundberg H 2002a Astron. Astrophys. 381 1090-3

Nilsson H, Ljung G, Lundberg H and Nielsen K E 2006 Astron. Astrophys. 445 1165-8

Nilsson H, Zhang Z G, Lundberg H, Johansson S and Nordstrom B 2002b Astron. Astrophys. 382 368-77

Nishikawa K I, Niemiec J, Hardee P E, Medvedev M, Sol H, Mizuno Y Zhang B, Pohl M, Oka M and Hartmann D H 2009 Astrophys. J. 698 L10-L13

Nishimura S et al 2011 Phys. Rev. Lett. 106052502

Nishino H et al 2009 Phys. Rev. Lett. 102141801

Nitz D, Kunau A E, Wilson K L and Lentz L R 1999 Astrophys. J. Suppl. Ser. 122 557-61

Nollett K M and Burles S 2000 Phys. Rev. D 61123505

Nuevo M, Milam S N, Sandford S A, Elsila J E and Dworkin J P 2009 Astrobiology 9 683-95

Öberg K I, van Dishoeck E F and Linnartz H 2009a Astron. Astrophys. 496 281-93

Öberg K I, Garrod R T, van Dishoeck E F and Linnartz H 2009b Astron. Astrophys. 504 891-913

Olive K A 1999 arXiv:astro-ph/9901231
Oliver P and Hibbert A 2010 J. Phys. B: At. Mol. Opt. Phys. 43074013

Osterbrock D E and Ferland G J 2006 Astrophysics of Gaseous Nebulae and Active Galactic Nuclei 2nd edn (Mill Valley, CA: University Science Press)

Otranto S and Olson R E 2011 Phys. Rev. A 83032710

Palmeri P, Quinet P, Wyart J-F and Biémont E 2000 Phys. Scr. $61323-34$

Palomares C 2009 Double-Chooz neutrino experiment EPS-HEP 2009: 2009 Europhysics Conf. on High Energy Physics (Krakow, Poland, 16-22 July 2009) p 275

Pascoli G and Polleux A 2000 Astron. Astrophys. 359 799-810

Patel H H and Ramsey-Musolf M J 2011 arXiv:1101.4665

Patel N A et al 2011 Astrophys. J. Suppl. Ser. 19317

Persson C M et al 2010 Astron. Astrophys. 521 L45

Pessah M E 2010 Astrophys. J. 716 1012-27

Pessah M E and Goodman J 2009 Astrophys. J. 698 L72-L76

Petrignani A et al 2009 J. Phys.: Conf. Ser. 192012022

Pettini M, Zych B J, Murphy M T, Lewis A and Steidel C S 2008 Mon. Not. R. Astron. Soc. 391 1499-510

Pfeiffer B, Kratz K-L, Thielemann F-K and Walters W B 2001 Nucl. Phys. A 693 282-324

Piekarewicz J 2010 J. Phys. G: Nucl. Part Phys. 37064038

Pierre Auger Collaboration 2010 Phys. Lett. B 685 239-46

Pietroni M 1993 Nucl. Phys. B 402 27-45

Pilaftsis A 2005 Phys. Rev. Lett. 95081602

Pilaftsis A 2009 J. Phys.: Conf. Ser. 171012017

Pilaftsis A and Underwood T E J 2005 Phys. Rev. D 72113001

Piran T 1999 Phys. Rep. 314 575-667

Polehampton E T, Menten K M, van der Tak F F S, White G J 2010 Astron. Astrophys. $\mathbf{5 1 0}$ A80

Poludnenko A Y, Dannenberg K K, Drake R P, Frank A, Knauer J, Meyerhofer D D, Furnish M, Asay J R and Mitran S 2004 Astrophys. J. 604 213-21

Porter R L, Ferland G J and MacAdam K B 2007 Astrophys. J. $657327-37$

Porter R L, Ferland G J, MacAdam K B and Storey P 2009 Mon. Not. R. Astron. Soc. 393 L36-L40

Porter S B, Desch S J and Cook J C 2010 Icarus 208 492-8

Pospelov M, Ritz A and Voloshin M B 2008 Phys. Lett. B 662 53-61

Pounds K A, Reeves J N, O'Brian P T, Page K A, Turner M J L and Nayakshin S 2001 Astrophys. J. 559 181-6

Pounds K A, Reeves J N, King R A and Page K L 2004 Mon. Not. R. Astron. Soc. 350 10-20

Prager S C, Rosner R, Ji H T and Cattaneo F 2010 Research Opportunities in Plasma Astrophysics (Princeton, NJ: Princeton Plasma Physics Laboratory) http://www.pppl.gov/conferences/2010/WOPA/index.html

Profumo S 2008 arXiv:0812.4457

Profumo S, Ramsey-Musolf M J and Shaughnessy G 2007 J. High Energy Phys. JHEP08(2007)010

Pudritz R E, Ouyed R, Fendt C and Brandenburg A 2007 Protostars and Planets $V$ ed B Reipurth et al (Tucson, AZ: University of Arizona Press) pp 277-94

Pulliam R L, Savage C, Agúndez M, Cernicharo J, Guélin M and Ziurys L M 2010 Astrophys. J. 725 L181-L184

Puget J L and Léger A 1989 Annu. Rev. Astron. Astrophys. 27 161-98

Qian Y-Z and Wasserburg G J 2007 Phys. Rep. 442 237-68

Quinet P, Palmeri P, Biemont E, McCurdy M M, Rieger G, Pinnington E H Wickliffe M E and Lawler J E 1999 Mon. Not. R. Astron. Soc. 307 934-40

Raiteri C M, Gallino R, Busso M, Neuberger D and Käppeler F 1993 Astrophys. J. $\mathbf{5 4 2}$ 400-3

Rapaport J and Sugarbaker E 1994 Annu. Rev. Nucl. Part. Sci. 44 109-53

Raymond J C, Wallerstein G and Balick B 1991 Astrophys. J. $383226-32$ 
Redman M P, Viti S, Cau P and Williams D A 2003 Mon. Not. R. Astron. Soc. 345 1291-6

Reighard A B et al 2006 Phys. Plasmas 13082901

Reipurth B and Bally J 2001 Annu. Rev. Astron. Astrophys. 39 403-55

Remijan A J, Hollis J M, Lovas F J Cordiner M A, Millar T J, Markwick-Kemper A J and Jewell P R 2007 Astrophys. J. 664 L47-L50

Remington B A, Drake R P and Ryutov D D 2006 Rev. Mod. Phys. $78755-807$

Ren Y, Yamada M, Ji H, Dorfman S, Gerhardt S P and Kulsrud R M 2008 Phys. Plasmas 15082113

Ren Y, Almagri A F, Fiksel G, Prager S C, Sarff J F and Terry P W 2009 Phys. Rev. Lett. 103145002

Rest A et al 2008 Astrophys. J. $6801137-48$

Reynolds S P, Borkowski K J, Hwang U, Hughes, J P, Badenes C, Laming J M and Blondin J M 2007 Astrophys. J. 668 L135-L138

Ricketts C, Contreras C, Walker R and Salama F 2011 Int. J. Mass Spectrom. 300 26-30

Richardson J E, Melosh H J, Lisse C M and Carcich B 2007 Icarus $190357-90$

Ritchey A M, Federman S R, Sheffer Y and Lambert D L 2011 Astrophys. J. 72870

Roberts H, Herbst E and Millar T J 2004 Astron. Astrophys. 424 905-17

Robey H F, Perry T S, Klein R I, Kane J O, Greenough J A and Boehly T R 2002 Phys. Rev. Lett. 89085001

Rogers F J and Iglesias C A 1994 Science 263 50-5

Rosen P A, Foster J M, Wilde B H, Hartigan P, Blue B E, Hansen J F, Sorce C, Williams R J R, Coker R and Frank A 2009 Astrophys. Space Sci. 322 101-5

Rosner R and Hammer D A 2010 Basic Research Needs for High Energy Density Laboratory Physics (Washington, DC: US Department of Energy) http://www.science.energy.gov/fes/news-and -resources/workshop-reports/

Rothman L S et al 2005 J. Quant. Spectrosc. Radiat. Transfer 96 139-204

Rubbia A 2011 March 16 talk 15th Int. Workshop on Neutrino Telescopes (Rome, 15-18 Mar. 2011) http://agenda.infn.it/ conferenceOtherViews.py?view $=$ standard $\&$ confId $=3101$

Runkle R C, Champagne A E, Angulo C, Fox C, Iliadis C, Longland R and Pollanen J 2005 Phys. Rev. Lett. 94082503

Ryan S G, Beers T C, Olive K A, Fields B D and Norris J E 2000 Astrophys. J. 530 L57-L60

Ryu D and Vishniac E T 1991 Astrophys. J. 368 411-25

Ryutov D D, Drake R P, Kane J, Liang E, Remington B A and Wood-Vasey M 1999 Astrophys. J. 518 821-32

Sakai N, Shiino T, Hirota T, Sakai T and Yamamoto S 2010 Astrophys. J. 718 L49-L52

Sako M et al 2003 Astrophys. J. 596 114-28

Salama F 1998 Solar System Ices ed B Schmitt et al (Dordrecht: Kluwer) pp 259-80

Salama F 1999 Solid Interstellar Matter: The ISO Revolution ed L d Hendecourt et al (Berlin: Springer) pp 65-87

Salama F 2008 IAU Symp. 251: Organic Matter in Space ed S Kwok and S A Sanford (Cambridge: Cambridge University Press) pp 357-66

Salama F, Galazutdinov G, Krelowski J, Biennier L, Beletsky Y and Song I 2011 Astrophys. J. 728 154-62

Saltzberg D, Gorham P, Walz D, Field C, Iverson R, Odian A, Resch G, Schoessow P and Williams D 2001 Phys. Rev. Lett. $862802-5$

Sandford et al 2006 Science 314 1720-4

Sargent B A et al 2009 Astrophys. J. 690 1193-207

Sarre P J 1980 J. Chim. Phys. Phys.-Chim. Biol. 77 769-71

Sarre P J 2006 J. Mol. Spectrosc. 238 1-10

Sasselov D D 2003 Astrophys. J. 596 1327-31
Sako M et al 2003 Astrophys. J. 596 114-28

Saumon D and Guillot T 2004 Astrophys. J. 609 1170-80

Savage C, Apponi A J and Ziurys L M 2004 Astrophys. J. 608 L73-L76

Savin D W et al 2011 arXiv:1103.1341

Schartman E, Ji H, Burin M and Goodman J 2011 arXiv:1102.3725

Schectman R M, Cheng S, Curtis L J, Federman S R, Fritts M C and Irving R E 2000 Astrophys. J. 419 207-23

Schilke P et al 2010 Astron. Astrophys. 521 L11

Schippers S 2009 J. Phys.: Conf. Ser. 163012001

Schippers S, Lestinsky M, Müller A, Savin D W, Schmidt E W and Wolf A 2010 Int. Rev. At. Mol. Phys. 1 109-20

Schlemmer S, Asvany O, Hugo E and Gerlich D 2006 Astrochemistry: Recent Successes and Current Challenges ed D C Lis et al (Cambridge: Cambridge University Press) p 125

Schmidt E W et al 2006 Astrophys. J. 641 L157-L160

Schmidt E W et al 2008 Astron. Astrophys. 492 265-75

Schoier F L, Maercker M, Justtanout K, Olofsson H, Black J H, Decin L, de Koter A, Waters R 2011 Astron. Astrophys. 530 A 83

Seager S, Richardson L J, Hanse B M S, Menou K, Cho Y Y-K and Deming D 2005 Astrophys. J. 632 1122-31

Sellgren K 1984 Astrophys. J. 277 623-33

Sellgren K, Werner M W, Ingalls J G, Smith J D T, Carleton T M and Joblin C 2010 Astrophys. J. 722 L54-L57

Shelton J and Zurek K M 2010 Phys. Rev. D 82123512

Silk J 1999 The relationship between the bright and dark matter The 3rd Stromlo Symposium: The Galactic Halo (ASP Conf. Ser. vol 165) ed B K Gibson et al (San Francisco, CA: Astronomical Society of the Pacific) pp 27-33

Silva L O, Fonseca R A, Tonge J W, Dawson J M, Mori W B and Medvedev M V 2003 Astrophys. J. 596 L121-L124

Simon M C et al 2010 Phys. Rev. Lett. 105183001

Sims I R 2006 Astrochemistry: Recent Successes and Current Challenges ed D C Lis et al (Cambridge: Cambridge University Press) p 97

Singh B S, Hass M, Nir-El Y and Haquin G 2004 Phys. Rev. Lett. 93262503

Sisan D R, Mujica N, Tillotson W A, Huang Y M, Dorland W, Hassam A B, Antonsen T M and Lathrop D P 2004 Phys. Rev. Lett. 93114502

Smith N and Morse J A 2004 Astrophys. J. 605 854-63

Smith R K, Chen G-X, Kirby K P and Brickhouse N S 2009 Astrophys. J. 700 679-83

Smith R K, Chen G-X, Kirby K P and Brickhouse N S 2009 Astrophys. J. 7012034 (erratum)

Sneden C, Cowan J J and Gallino R 2008 Annu. Rev. Astron. Astrophys. 46 241-88

Sneden C, Lawler J E, Cowan J J, Ivans I I and den Hartog E A 2009 Astrophys. J. Suppl. Ser. 182 80-96

Snow T P and McCall B J 2006 Annu. Rev. Astron. Astrophys. 44 367-414

Sobeck J S, Lawler J E and Sneden C 2007 Astrophys. J. 667 1267-82

Soderberg A M et al 2008 Nature 453 469-74

Sofia U J, Meyer D M and Cardelli J A 1999 Astrophys. J. 522 L137-L140

Sogoshi N, Kato Y, Wakabayashi T, Momose T, Tam S, DeRose M E and Fajardo M E 2000 J. Phys. Chem. A 104 3733-42

Spence E J, Nornberg M D, Jacobson C M, Parada C A, Taylor N, Kendrick R D and Forest C B 2007 Phys. Rev. Lett. 98164503

Spitkovsky A 2008 Astrophys. J. 673 L39-L42

Springel V et al 2005 Nature 435 629-36

Springer P T et al 1997 J. Quant. Spectrosc. Radiat. Transfer 58 927-35

Steenbrugge K C, Kaastra J S, de Vries C P and Edelson R 2003 Astron. Astrophys. $\mathbf{4 0 2}$ 477-86 
Steenbrugge K C, Kaastra J S, Sako M, Branduardi-Raymont G, Behar E, Paerels F B S, Blustin A J and Kahn S M 2005 Astron. Astrophys. 432 453-62

Stefani F, Gundrum T, Gerbeth G, Rudiger G, Schultz M, Szklarski J and Hollerbach R 2006 Phys. Rev. Lett. 97184502

Steigman G 2011 Primordial nucleosynthesis: the predicted and observed abundances and their consequences NIC XI: Proc. 11th Symp. on Nuclei in the Cosmos (Heidelberg, Germany, 11-23 July 2010) 001

Stenrup M, Larson Å and Elander N 2009 Phys. Rev. A 79012713

Storey P J and Hummer D G 1995 Mon. Not. R. Astron. Soc. 272 41-8

Strieder F et al 2001 Nucl. Phys. A 696219

Su M, Slatyer T R and Finkbeiner D P 2010 Astrophys. J. 724 1044-82

Sun X, Intrator T P, Dorf L, Sears J, Fumo I and Lapenta G 2010 Phys. Rev. Lett. 105255001

Symes D R et al 2010 High Energy Density Phys. 6 274-9

Tangri V, Terry P W and Fiksel G 2008 Phys. Plasmas 15112501

Tenenbaum E D and Ziurys L M 2008 Astrophys. J. 680 L121-L124

Tenenbaum E D and Ziurys L M 2010 Astrophys. J. 712 L93-L97

Tenenbaum E D, Woolf N J and Ziurys L M 2007 Astrophys. J. 666 L29-L32

Tenenbaum E D, Milam S N, Woolf N J and Ziurys L M 2009 Astrophys. J. 704 L108-L112

Tenenbaum E D, Dodd J L, Milam S N, Woolf N J and Ziurys L M 2010a Astrophys. J. 720 L102-L107

Tenenbaum E D, Dodd J L, Milam S N, Woolf N J and Ziurys L M 2010b Astrophys. J. Suppl. Ser. 190 348-417

Terzieva R and Herbst E 2000 Int. J. Mass Spectrom. 201 135-42

Thaddeus P, Gottlieb C A, Gupta H, Brünken S, McCarthy M C, Agúndez M, Guélin M and Cernicharo J 2008 Astrophys. J. 677 1132-9

The L-S, El Eid M F and Meyer B S 2007 Astrophys. J. $6551058-78$

Tielens A 2005 The Physics and Chemistry of the Interstellar Medium (Cambridge: Cambridge University Press)

Tikhonchuk V T et al 2008 Plasma Phys. Control. Fusion 50124017

Tinetti G et al 2007 Nature 448 169-71

Tom B A et al 2009 J. Chem. Phys. 130031101

Tornow W, Czakon N G, Howell C R, Hutcheson A, Kelley J H, Litvinenko V N, Mikhailov S F, Pinayev I V, Weisel G J, and Witala H 2003 Phys. Lett. B 574 8-13

Torres G et al 2011 Astrophys. J. 72724

Trotta R, Feroz F, Hobson M P, Roszkowski L and Ruiz de Austri R 2008 J. High Energy Phys. JHEP12(2008)024

Trujillo C A, Brown M E, Barkume K M, Schaller E L and Rabinowitz D L 2007 Astrophys. J. 655 1172-8

Tucker-Smith D and Weiner N 2001 Phys. Rev. D 64043502

van Boekel R, Min M, Waters L B F M, de Koter A, Dominik C, van den Ancker M E, Bouwman J 2005 Astron. Astrophys. 437 189-208 van der Holst B, Toth G, Sokolov I V, Powell K G, Holloway J P, Myra E S, Stout Q, Adams M L, Morel J E and Drake R P 2011 Astrophys. J. Suppl. Ser. 19423

van Veelen B, Langer N, Vink J, Garcia-Segura G and van Marle A J 2009 Astron. Astrophys. 503 495-503

Vastel C, Caselli P, Ceccarelli C, Phillips T, Wiedner M C, Peng R, Houde M and Dominik C 2006 Astrophys. J. $6451198-211$

Vernazza J E, Avrett E H and Loeser R 1976 Astrophys. J. Suppl. Ser. 30 1-60

Vishniac E T 1983 Astrophys. J. 274 152-67

Walker K M, Federman S R, Knauth D C and Lambert D L 2009 Astrophys. J. 706 614-22

Walsh C, Harada N, Herbst E and Millar T J 2009 Astrophys. J. $700752-61$

Wang F L, Fujioka S, Nishimura H, Kato D, Li Y T, Zhao G, Zhang $\mathrm{J}$ and Takabe H 2008 Phys. Plasmas 15073108

Wang L, Howell D A, Höflich P and Wheeler J C 2001 Astrophys. J. $5501030-5$

Wang X K, Lin X W, Mesleh M, Jarrold M F, Dravid V P, Ketterson J B and Chang R P H 1995 J. Mater. Res. 10 1977-38

Watson W D 1973 Astrophys. J. 183 L17-L20

Waxman E 2006 Plasma Phys. Control. Fusion 48 B137-B151

Westmoquette M S, Exter K M, Smith L J, and Gallagher J S 2007 Mon. Not. R. Astron. Soc. 381 894-912

Whittet D C B 1997 Orig. Life Evol. Biosph. 27 249-62

Whittet D C B 2003 Dust in the Galactic Environment 2nd edn (Bristol: Institute of Physics)

Wickliffe M E and Lawler J E 1997 J. Opt. Soc. Am. B $14737-53$

Wickliffe M E, Lawler J E and Nave G 2000 J. Quant. Spectrosc. Radiat. Transfer 66 363-404

Wiescher M, Gorres J, Uberseder E, Imbriani G and Pignatari M 2010 Annu. Rev. Nucl. Part. Sci. 60 381-404

Wood K and Raymond J C 2000 Astrophys. J. 540 563-71

Xu H L, Svanberg S, Quinet P, Garnir H P and Biémont E 2003 J. Phys. B: At. Mol. Opt. Phys. 36 4773-87

Yamada M 2007 Phys. Plasmas 14058102

Yamada M, Kulsrud R and Ji H 2010 Rev. Mod. Phys. 82 603-64

Yirak K, Frank A, Cunningham A and Mitran S 2008 Astrophys. J. 672 996-1005

Yirak K, Frank A and Cunningham A 2012 Astrophys. J. at press (arXiv:1101.6020)

Young K, Cox P, Huggins P J, Forveille T and Bachiller R 1999 Astrophys. J. 522 387-96

Zack L N, Ziegler N and Ziurys L M 2012 Astrophys. J. submitted

Zatsepin G T and Kuz'min V A 1966 JETP Lett. 4 78-80

Zheng W J, Jewitt D and Kaiser R I 2009 J. Phys. Chem. A $11311174-81$

Ziurys L M 2006 Proc. Natl Acad. Sci. USA 103 12274-9

Zolensky et al 2006 Science 314 1735-9

Zweibel E G and Yamada M 2009 Annu. Rev. Astron. Astrophys. 47 292-332 